\title{
Catalyst development for an arc-ignited hydrogen peroxide/ABS hybrid rocket system
}

\begin{abstract}
The authors have collaborated with an industry partner to develop a prototype upper stage for a dedicated nano-launch vehicle. In addition to providing sufficient impulse for orbit insertion, the unique motor system also provides capability for multiple restarts; allowing operation as an orbital maneuvering thruster. The hybrid motor design uses $85 \%-90 \%$ hydrogen peroxide solution and 3-D printed ABS (acrylonitrilebutadiene-styrene) as propellants. In the original system design the peroxide catalyst bed was completely removed and a patented arc-ignition system thermally ignited the propellants. The thermal ignition system was effective but resulted in a combustion latency of approximately 1 -second, reducing overall performance and allowing for significant variability in the delivered total impulse. This work investigates whether adding a small catalyst pack for ignition augmentation can eliminate or significantly reduce the observed ignition latency and improve overall system performance. The effectiveness of multiple catalytic minerals including potassium permanganate, manganese dioxide, manganese (III) oxide, and potassium nitrate were examined and compared to traditional noble-metal catalyst materials like silver or platinum. These alternative materials are significantly less expensive than noble metals. Catalytic activity test results, designs for an augmentation catbed integrated with the thermal ignition system, and preliminary unaugmented and augmented hot-fire test results are presented.
\end{abstract}

Volume 2 Issue $6-2018$

Stephen A Whitmore,' Christopher J

Martinez,' Daniel P Merkley²

'Professor, Mechanical and Aerospace Engineering Department, Utah State University, USA

${ }^{2}$ Graduate Research Assistant, Mechanical and Aerospace

Engineering Department, Utah State University, USA

Correspondence: Stephen A Whitmore, Professor, Mechanical and Aerospace Engineering Department, Utah State University, Logan Utah, 84322-41 30, USA, Tel 435-797-295 I

Email stephen.whijmore@usu.edu

Received: October 05, 2018 | Published: November 23, 2018

\section{Nomenclature}

$\begin{array}{lll}A & = & \begin{array}{l}\text { Arrhenius equation scale factor, } \mathrm{mol} / \mathrm{sec} \\ A_{c} \\ \text { catbed, } m^{2}\end{array} \\ \begin{array}{c}A_{\text {screen }} \\ \mathrm{cm}^{2}\end{array} & = & \text { geometric cross-sectional area of the } \\ A_{\text {sshp }}= & \text { surface area of sphere with equivalent volume to }\end{array}$
test specimen, $\mathrm{cm}^{2}$

$$
A_{\text {wire }}=
$$$$
\text { projected 2-D wire area in screen mesh, } \mathrm{cm}^{2}
$$$$
\mathrm{CSI}=
$$$$
\text { contact surface index, } \mathrm{mm}^{2}
$$

$\mathrm{cm})$

$$
D_{\text {alumina }}
$$$$
=
$$

alumina pellet diameter, $1 / 8$ in $(0.3175$

$D_{\text {pellet }}$

$$
=
$$$$
\text { effective diameter of coated pellet, } \mathrm{cm}
$$

$D_{\text {wire }}=$ wire strand diameter, $\mathrm{cm}$

$d_{p}$ bed, $c m$

$$
\begin{array}{lll}
f & = & \text { peroxide mass concentration } \\
f, & = & \text { effective mass concentration for }
\end{array}
$$
inefficient decomposition

$$
\begin{array}{lll}
E_{a} & = & \text { energy of activation, } \mathrm{kJ} / \mathrm{mol} . \\
\Delta H_{d}= & & \text { peroxide enthalpy of decomposition, } \mathrm{J} / \mathrm{g} \\
I_{s p} & = & \text { specific impulse, } s
\end{array}
$$
$k_{r}$
$=$
rate of reaction, $M / s$
$L \quad=$
length of catalyst bed active components,
$L_{\text {alumina }} \quad=\quad$ alumina pellet length, $1 / 4$ in $(0.635 \mathrm{~cm})$
$L_{c} \quad=\quad$ catalyst pack length, $m$
$L_{\text {pellet }}=\quad$ effective length of coated pellet, $\mathrm{cm}$
$L_{\text {wire }}=\quad$ effective wire strand length, $\mathrm{cm}$

$\mathrm{cm}$

$M \quad=\quad$ Screen mesh number-number of empty areas per linear inch wire mesh, in ()

$\delta m_{O 2}=$ mass of oxygen released from peroxide decomposition, $g$

$$
\begin{array}{lll}
M_{W} & = & \text { molecular weight, } J / \mathrm{kg}-K \\
\mathbb{M}= & \text { solution molality, moles } / \mathrm{g}
\end{array}
$$

$\mathbf{M}=$ solution molarity, mole/liter

$m \quad=\quad$ residual mass of reactant or general mass quantity, $g$

$\begin{array}{llll}\dot{m} & = & \text { mass flow rate, } \mathrm{kg} / \mathrm{s} & \\ m_{p} & = & \text { particle mass, } \mathrm{g} & \\ \mathrm{n} & = & \text { number of moles } & \\ P_{c} & = & \text { combustion chamber } & \text { pressure }\end{array}$
downstream of injector, $\mathrm{kPa}$
$P_{\text {exit }}=$
catbed exit pressure upstream of injector, $\mathrm{kPa}$
$\delta P$ 
$\delta P_{\mathrm{O}_{2}}=\quad$ pressure change due to oxygen released from peroxide decomposition, $\mathrm{kPa}$

$\begin{array}{cll}\begin{array}{cll}R_{u} \\ m o l-K\end{array} & = & \text { universal gas constant, } 8314.4612 \mathrm{~J} / \\ R_{g} & = & \text { gas constant for oxygen, } \mathrm{J} / \mathrm{kg}-\mathrm{K} \\ \mathrm{S} & = & \text { Arrhenius equation curve-fit, slope } \\ S & = & \text { variable of integration, } \mathrm{cm} \\ T & = & \text { temperature of reaction, } K \\ T_{\text {exit }}= & \text { catbed exit temperature, }{ }^{\circ} \mathrm{C} \\ T_{\text {final }}= & \text { catbed exit temperature at rn valve closure during } \\ \text { warm flow test, }{ }^{\circ} \mathrm{C}\end{array}$
warm flow test, ${ }^{\circ} \mathrm{C}$

$\begin{array}{lll}T_{0} & = & \text { combustion chamber temperature, } K \\ V & = & \text { catalyst bed internal flow volume, } \mathrm{cm}^{3} \\ V_{c} & = & \text { ullage volume of reaction chamber, } \mathrm{cm}^{3} \\ V_{p} & = & \text { particle volume, } \mathrm{cm}^{3} \\ V_{\text {wire }}= & \text { volume of a wire strand, } \mathrm{cm}^{3} \\ W & = & \text { empty area between wire strands in a }\end{array}$

mesh.

$Y_{0}$

$\Delta E \quad=$

= longitudinal distance dome catbed, $\mathrm{cm}$

$\Delta t^{a} \quad=$

$\Delta x \quad=$

$\eta \quad=$

$\eta^{*} \quad=$

$\phi \quad=$

$\phi$

open to geometric internal flow volume.

$\begin{array}{lll}\rho & = & \text { material density, } \mathrm{g} / \mathrm{cm}^{3} \\ \rho_{\text {soln }}= & \text { total peroxide solution density, } \mathrm{g} / \mathrm{cm}^{3} \\ \mu & = & \text { viscosity, } \mathrm{Pa}-\mathrm{S}\end{array}$

\section{Introduction}

Although the market for commercially designed and manufactured small spacecraft has grown enormously during the last decade, technology development has mostly centered on spacecraft bus design and miniaturization of sensor components. The space-launch industry has generally not kept pace with the growth trend. With the current state-of-the art, the only available for small commercial, commercial or academic payloads to reach orbit is by "rideshare." A "rideshare" is defined as a secondary payload, smaller in size and weight than the primary payload, that is placed into orbit by a launch vehicle that is mostly paid for by the primary sponsor. Because the sponsor pays the lion's share of the launch costs, the secondary payload receives a substantially reduced launch price in exchange for trade-offs with regard to the payload's final orbit and launch schedule. These tradeoffs typically include having little or no control over the launch date and time, the final orbital parameters, or the ability to halt the launch and remove the payload should a payload failure occur during ground processing prior to launch.

Only a few specialized launch vehicles have upper stages with the ability for in-space restarts; these are typically reserved for expensive government-owned reconnaissance, communications, or command \& control satellites. For existing rideshare launch opportunities, nano-scale spacecraft are delivered to orbit as passive payloads and must accept whatever orbit they achieve during the deployment process. Currently, "rideshare" services are primarily provided by the DoD/NASA Evolved Expendable Launch Vehicle (EELV) using its secondary payload adapter (ESPA). ${ }^{1}$ SpaceX has also offered some secondary payload slots on its Falcon 9 launches; but these opportunities are limited. ${ }^{2}$ So unfortunately, with the current state of world launch capability, inadequate low earth orbit (LEO) "rideshare" launch opportunities are available to accommodate the demand for these NanoSat demonstration missions. Secondary payloads, especially in the nanosat class, with little or no ability determine their deployment orbit, will remain a novelty with little means to accomplish serious scientific, strategic, or commercial missions.

Finally, even when flown as secondary payloads, NanoSat launch costs are extremely high and are commercially prohibitive. Current launch costs are high for a variety of reasons, with human and environmental safety concerns being very high on this list. Clearly, a dedicated Nanosat launch vehicle is required to reduce costs and provide the required LEO access for NanoSats to achieve practical utility. This vehicle design must address the issues that make traditional launch costs so prohibitive. The activity to be reported by this paper was initiated in response to the above-described nanolaunch challenge. Restartable, high performing launch vehicle stages in the $25-250 \mathrm{~kg}$ payload size class are not currently commercially available. The proposed hybrid "green-propellant" systems offer significant advantages over competing technologies in the areas of cost, safety, and mission capability. The resulting technology will fulfill the ever-growing mission demands of the extensive NanoSat market by enabling dedicated launch for CubeSat scale payloads.

\section{Hybrid rockets as a green propulsion alternative}

The inherent safety and environmental friendliness of hybrid rocket systems have been known for several decades. ${ }^{1}$ Hybrids have the potential to act as an ideal "green" alternative to hydrazine. Historically, due to the lack of a reliable non-pyrotechnic, multiple-use ignition method, hybrid rockets have never been seriously considered as feasible for in-space propulsion. However, as will described later in Section III of this paper, this issue has been overcome by leveraging the unique electrical breakdown properties of certain 3-D printed thermoplastics. ${ }^{2}$ The associated arc-ignition concept has been developed into a power-efficient ignition system that can be started, stopped, and restarted with a high degree of reliability. Hybrid rockets offer particular utility for the upper stages of a nano-launch vehicle. Although a hybrid rocket will increase the overall system dry mass compared to a solid-propellant motor, the capabilities to throttle, shutdown on demand, coast, and relight the motor, will the offset any lost in performance of the stage. Such a "smart-stage" would not only provide $\triangle \mathrm{V}$ to enable the payload to reach orbit; but can also serve

${ }^{1}$ Perry B. ESPA: An Inexpensive Ride to Space for Secondary Payloads. Milsat Magazine; 2012.

${ }^{2}$ Foust J. New Opportunities for Smallsat Launches. The Space Review; 2011. 
as an on orbit maneuvering system that allows precise placement of the payload. Finally, orbital debris due to abandoned spacecraft is becoming a huge issue. It likely that United Nations regulations will soon demand active end-of-lifetime deorbit. The system to be investigated here may also provide on-orbit maintenance or end-oflifetime de-orbit capability. ${ }^{3}$

Previously, the authors of this report have experienced considerable success with small spacecraft systems using gaseous oxygen $(G O X)$ and 3-D printed thermoplastics like ABS (acrylonitrile-butadienestyrene) as the propellants. ${ }^{4,5}$ However, GOX was rejected for this upper-stage application due to its low density, required storage pressures, and significant potential for fire hazards when stored in significant quantities at high pressures. Due to its high density, hydrogen peroxide $\left(\mathrm{H}_{2} \mathrm{O}_{2}\right)$ was considered to be very promising oxidizer for this application. In order to achieve comparable density to $\mathrm{H}_{2} \mathrm{O}_{2}$, GOX would need to be stored at pressures above 10,000 psi. During this investigation USU has built a prototype 1/4th scale motor and with a novel non-catalytic ignition system in order to gain experience with using peroxide in hybrid rocket system designs. This paper reports on the development and testing of that prototype system. Primary emphases are placed on ensuring reliable ignition, characterizing performance and enhancing the system performance, and developing regression rate curves. As occurs with other hybrid motors, the $\mathrm{H}_{2} \mathrm{O}_{2} / \mathrm{ABS}$ motor can throttle and execute multiple restarts. These features make it an excellent candidate for an upper stage launch vehicle due to its ability to give multiple and precise $\Delta v$ 's to enable the payload to achieve its desired orbit, and then make necessary maneuvers during its mission.

\section{Review of electrical arc-ignition technology}

The Propulsion Research Laboratory at Utah State University has recently initiated development of a novel one-quarter scale hybrid rocket stage-motor that employs medium grade hydrogen peroxide $(85 \%)$ and additively-manufactured ABS as propellants. ${ }^{6}$ The thruster system was adapted from previous "Green" hybrid systems designed and tested at USU. ${ }^{4}$ For this prototype motor, the hydrogen peroxide was directly injected into the fuel grain port using a commercial hollow-cone 50-degree nozzle to atomize the oxidizer. Using Utah State's patented Arc-Ignition system, the ABS was pyrolyzed by directing a high voltage through a 3D printed ignitor cap made of ABS. To initiate the decomposition of the hydrogen peroxide, a short pre-lead fire of GOX was used to warm the fuel grain, and raise the combustion chamber port prior to peroxide injection. This approach prevents super-cooling downstream of the liquid injection point, and also provides enough activation energy to start the decomposition process.

The alternative-ignition approach to be presented here leverages the patented arc-ignition system developed for hybrid rockets by Utah State University. ${ }^{7,8}$ The arc-ignition technology derives from the electrical breakdown properties of certain 3-D printed thermoplastics like Acrylonitrile Butadiene Styrene (ABS), Low Density Polyethylene (LDPE) and High-Impact-Polystyrene (HIPS). These properties were discovered serendipitously while investigating the thermodynamic performance of ABS as a hybrid rocket fuel. ${ }^{9}$ This arc-ignition concept has been engineered into a power-efficient system that can be started, stopped, and restarted with a high degree of reliability. On demand ignition has been demonstrated using compressed air, nitrous oxide $\left(\mathrm{N}_{2} \mathrm{O}\right),{ }^{10}$ gaseous oxygen, ${ }^{11}$ and hydrogen peroxide ${ }^{6}$ as oxidizers. The arc ignition process is extremely energy efficient, requiring as little as
$10 \mathrm{~W}$ for less than one second. Typical ignition energies are less than 10 Joules. The arc-ignition system has been demonstrated across a wide range ambient pressures including vacuum conditions. ${ }^{12}$

Figure 1 shows an additively manufactured example of an ABS fuel grain with built in ignitor geometry. Pictured are the precombustion chamber that features two impingement shelves intended to trap and mix the injected oxidizer with the pyrolyzed fuel. Two electrodes, insulated by industry standard ESC connectors, ${ }^{3}$ are embedded into the top face of the fuel grain. Dual redundant, solidcore copper wires are routed from the electrodes to small gaps located on the impingement shelves. The wires are insulated and protected from flame by 3-D printed circular "slots" that insert into the electrode wire gaps. The conducting paths terminate facing each other, flush with the combustion port surface, and exposed to the interior of the combustion chamber.

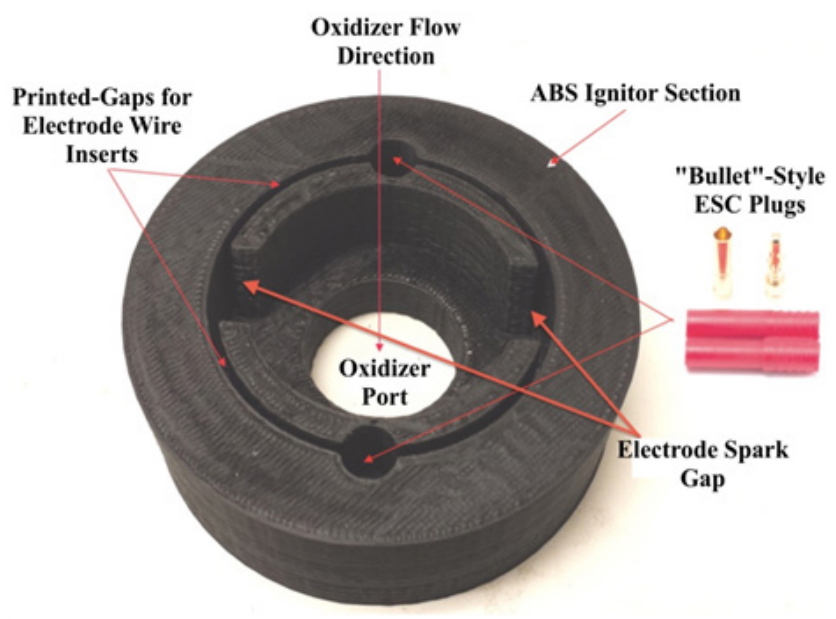

Figure I Additively-manufactured fuel grain with integrated electrode paths.

When an electrostatic potential is placed across the electrodes, charges flow from the energized electrode causing a localized electrostatic breakdown of the fuel material. This breakdown allows the charges to carve a path through the material. The resulting arctrack completes the circuit path to return electrode. ${ }^{13}$ Current flowing through the material causes Joule heating and pyrolyzes some of the material hydrocarbons. Ignition of the pyrolized fuel vapors occurs as soon as oxidizer flow is initiated. The initial arc-track can be pre-set by doping the surface with graphite powder. Once a surface arc-path has been set, graphite doping is no longer required. Depending on the arc-path length, typical impedances across the material between the wire leads lies in the range from $100 \mathrm{k} \Omega$ to $2.5 \mathrm{M} \Omega$. Identical ABS fuel segments made from extruded/machined ABS or similar thermoplastics do not exhibit the observed arcing properties. Thus, 3-D printing is an essential requirement of the system. With this system design very low energy inputs are required for the system ignition. Typical ignition electrical power inputs are less 5 watts for 1 second duration.

\section{Background on the thermal decomposition of $\mathrm{H}_{2} \mathrm{O}_{2}$ using arc-ignition system}

In this approach the peroxide flow is pre-lead by a small flow of gaseous oxygen injected into a combustion chamber lined with the 3-D printed ABS fuel. The arc-ignition system weakly initiates combustion

\footnotetext{
${ }^{3}$ Anon. Motor to ESC Connectors; 2017.
} 
between the injected oxygen and the fuel source, and is followed by the peroxide flow. Previous studies have demonstrated that GOX/ABS combustion generates temperatures exceeding $2800^{\circ} \mathrm{C}$, and specific enthalpies greater than $8.5 \mathrm{MJ} / \mathrm{kg}$. Thus, with the properly tuned GOX pre-lead massflow, there exists sufficient energy to decompose the incoming peroxide flow, while simultaneously initiating fulllength hybrid combustion. Once peroxide decomposition begins, then the additional energy of decomposition contributes to the overall combustion process. After the GOX pre-lead is terminated, Combustion is sustained by the oxygen liberated by the thermal decomposition of the peroxide.

\section{Additively-manufactured fuel grain design}

Previous arc-ignition test prototypes have manufactured the entire fuel grain using 3-D printing. However, due to the associated costs of 3-D printing and the large volume of material that would be required for this testing campaign, only a small ignitor cap section was 3-D printed for each grain. The printed cap was bonded to an extruded ABS rod, machined to fit the appropriate dimensions, to complete the fuel grain. With this change the majority of the fuel grain volume consisted of the significantly cheaper extruded material, as opposed to the 3-D printed material. This approach resulted in considerable cost savings with no reduction in performance. The small 3-D printed cap section provided more than sufficient fuel pyrolysis to seed the flow with hydrocarbon vapor and initiate combustion.

Figure 2 shows the fuel grain and thrust chamber design features. The thrust chamber was developed from a $76 \mathrm{~mm}$ hobby-rocket motor case with a graphite nozzle and retainer system. These components were well characterized during a previous research campaign. ${ }^{14}$ The ignitor grain segments were manufactured on a Stratasys Dimension $\mathbb{R}$ 1200 es 3D FDM 4 printer using their standard-grade ABS stock material. The electrodes were manually installed into the assembly and the fuel grains and sealed with the printed "slots" described earlier. The fuel grains were completed by bonding the 3-D printed caps into the lower extruded sections using commercial grade ABS pipe joint cement. The ignitor grain segments were printed fully dense to match the extruded ABS material density of approximately 0.970 $\mathrm{g} / \mathrm{cm}^{3}$. Each ignitor grain segment weighed approximately 110 grams. The ABS grains were machined down to fit the $76 \mathrm{~mm}$ chamber from a 3" diameter extruded rod. The bonded grains were press-fit inserted into the motor case with no insulating sleeve. The extruded ABS grain segments weighed approximately 700 grams each. Each completed fuel grain weighed approximately 810 grams.

\section{Peroxide and GOX injector designs}

Following guidance offered by Anthione et al., ${ }^{15}$ a hollow-cone injector swirl injector was used in order to effectively atomize the peroxide flow and provide for positive impingement against the fuel port walls. In order to evenly mix the ignitor GOX flow into the peroxide stream, a symmetrically-distributed GOX injector was machined into the motor cap. Figure 3 shows the motor and ignitor caps and the peroxide and GOX injector configurations. Both large and small port peroxide injectors were successfully tested. The small port configuration produced the greatest pressure drop and overall motor burn stability; however, it suffered from a significantly reduced burn lifetime compared to the larger injector.

${ }^{4}$ Anon. Dimension 1200es, Durability Meets Affordability.
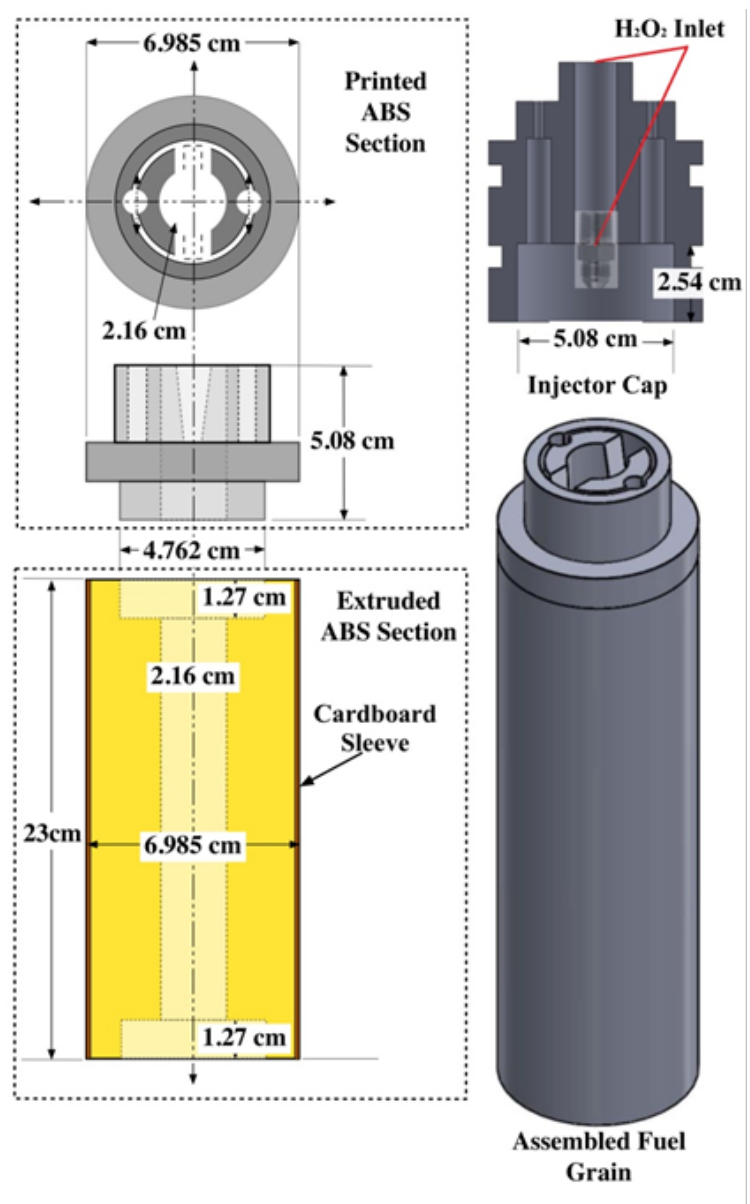

Figure 2 Schematic of 3-D printed ignitor, extruded ABS lower grain segment, and injector cap interface.

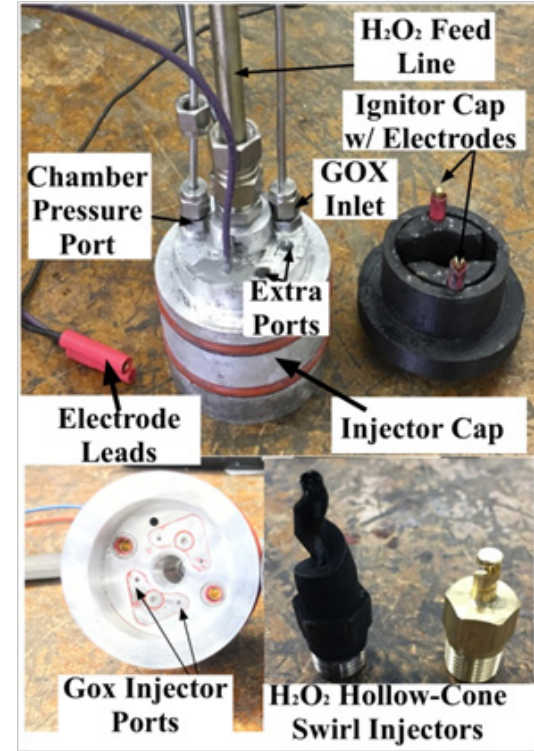

Figure 3 Injector cap, ignition cap, GOX injector, and peroxide injector designs. 


\section{Summary of initial Peroxide/ABS arc-ignition test results}

As described previously, the initial phase of the development campaign examined various hardware permutation in order to understand the configuration elements that were essential to establishing reliable and repeatable ignition sequences using only thermal ignition. ${ }^{16}$ During this testing campaign, the GOX valve and ignition spark were was set to pre-lead the peroxide valve opening by 2 seconds. The GOX valve would remain open for two seconds after the peroxide valve was opened. This approach allowed multiple full-ignitions to be reliably achieved. For later tests the GOX pre-lead was shortened to 1 seconds with no deleterious effects. Finally, the duration of GOX flow after the peroxide run opening, was shortened from two-seconds to one-second. This change did not significantly affect the ignition reliability. For all burns the peroxide valve was opened at time zero, and remained opened until all of the liquid in the 2-liter peroxide run tank was exhausted. In order to achieve a wider variety of massflux rates during this burn series, the peroxide injector feed pressure $p_{f}$ was varied by manually adjusting the regulator output over a range from 180 to $300 \mathrm{psig}$. The resulting chamber pressure varied over a range from 160 to 200 psia. Whitemore et al., ${ }^{6,16}$ describe the test apparatus and procedures in detail. All tests were performed at ambient pressure background conditions.

Figure 4 shows a typical arc-ignition time history where a 3-D printed ABS fuel grain is burned with an $88 \%$ hydrogen peroxide solution. Plotted are (a) directly measured (load cell) thrust and thrust calculated from chamber pressure using the De Laval flow equations, (b) chamber pressure, (c) GOX, peroxide, nozzle exit, and fuel massflow rates, (d) cumulative and instantaneous specific impulse values as calculated using both measured and calculated thrust levels, (e) input power and energy levels, and (f) the oxidizer and total massflux through the fuel port. For these tests a venturi flow meter was used to sense the oxidizer massflow, and the oxidizer massflow and massflux time history plots have been time-shifted to account for the transport latency caused by the pipe distance from the venturi outlet to the injector port.

In Figure 4, major feature of the massflow (c) and mass flux $(f)$ time history plots is the large initial spike in oxidizer massflow. This feature occurs immediately upon opening of the main peroxide run valve (at time zero), and precedes the buildup of full thrust (a) chamber pressure (b) by approximately $1 / 2$ second. The latency of the chamber pressure response results from the injection of liquid peroxide into the thrust chamber. This initial "wet" flow does not contribute to the chamber pressure buildup, and the nozzle exit remains initially unchoked. After a short period of "smoldering," sufficient energy is released by the GOX/ABS pre-burn to begin decomposition of the incoming peroxide. Once the chamber pressure builds up to exceed approximately two atmospheres of oxygen partial pressure, the nozzle chokes and the test experience of Whitemore et al., ${ }^{2}$ has demonstrated that full combustion will occur. For the tests reported by Whitemore et al., ${ }^{6}$ this ignition transient would typically vary between 400 and 800 milliseconds. The higher feed pressures of 250-00 psig tended to exhibit lower ignition latencies. The lower feed pressures of 180-200 psig resulted in larger ignition delays, with transients as long as 1.5 seconds observed on two tests.
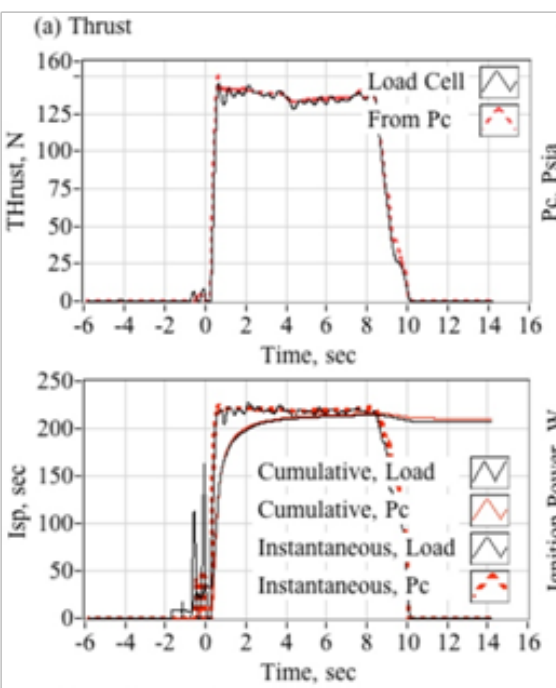

(d) Specific Impulse
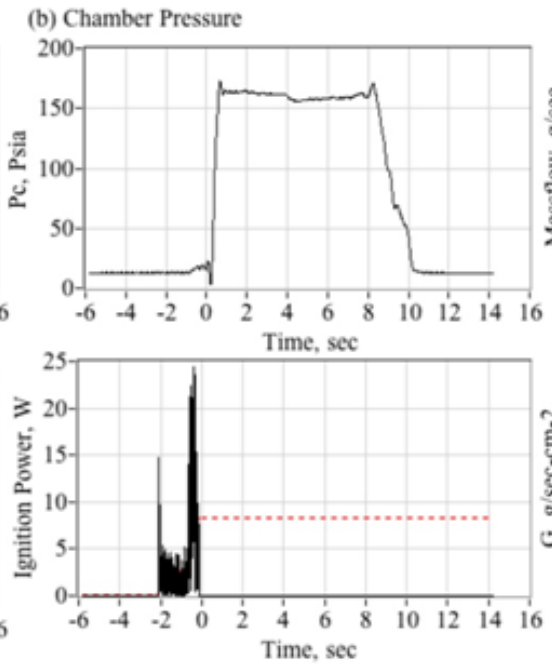

(e) Igniton Power, Total Energy Input
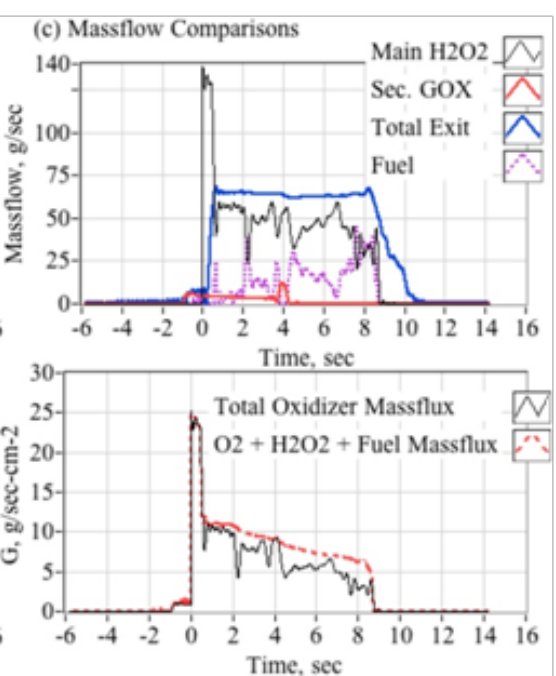

(f) Massflux, g/sec- $\mathrm{cm}^{\wedge} 2$

Figure 4 An 8-Second characterization burn using ABS fuel, and large-port hollow cone injector.

Note that the specific impulse plot (d) presents 4 graphs based upon the load-cell sensed thrust or thrust calculated from chamber pressure using theoretical values for the ratio of specific heats and molecular weight, and assuming a fully started nozzle. The cumulative calculation integrates the thrust over time and divides by the total consumed mass. The instantaneous calculation divides the instantaneous total massflow into the sensed or calculated thrust level. Note that the cumulative $I_{s p}$ is lower than the instantaneous value, this result is primarily due to the initial shot of unburned oxidizer that depletes total propellant mass but provides little to the overall thrust level. Figure 5 shows a series of still images of the ignition sequence. Note (a) the initial disperse flame as the GOX pre-lead ignites but does not choke the nozzle exit. This event is followed by (b) the wet peroxide flow, and a (c) very rich flow stream where the peroxide flow is in the process of fully decomposing. Finally, in (d) full combustion is achieved and a well-developed plume results. The elapsed time on each image begins from the time when the main valve opening command is sent. 


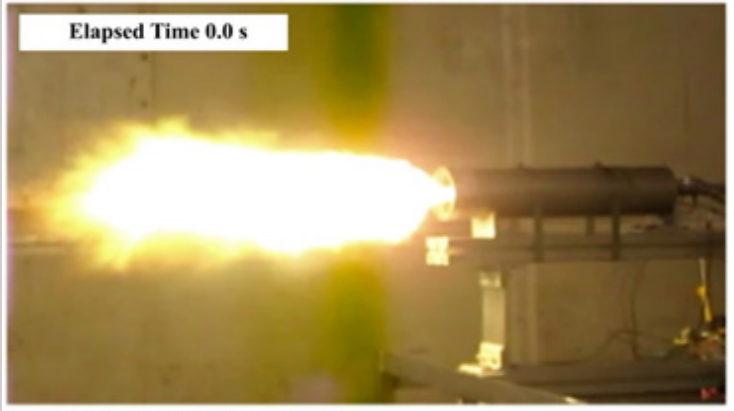

a) Ignition with GOX PreLead

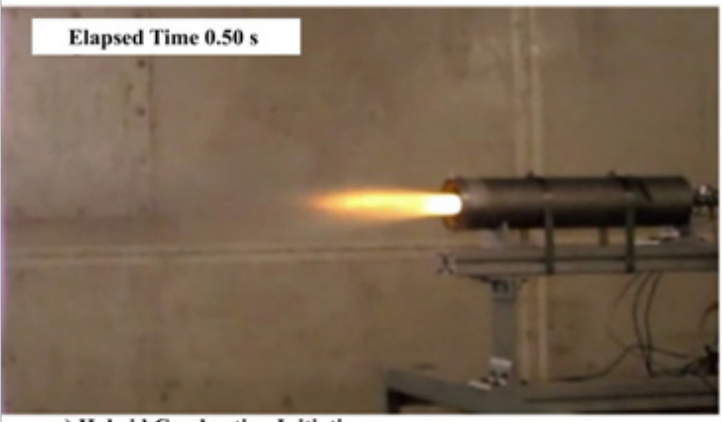

c) Hybrid Combustion Initiation

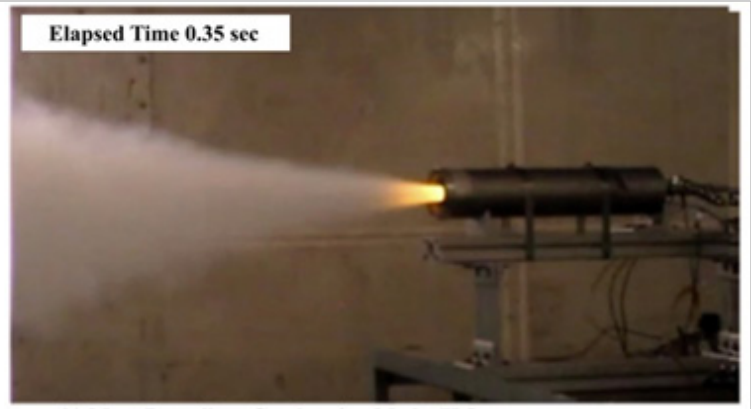

b) MonoPropellant Combustion Mode (H.O Decomposition)

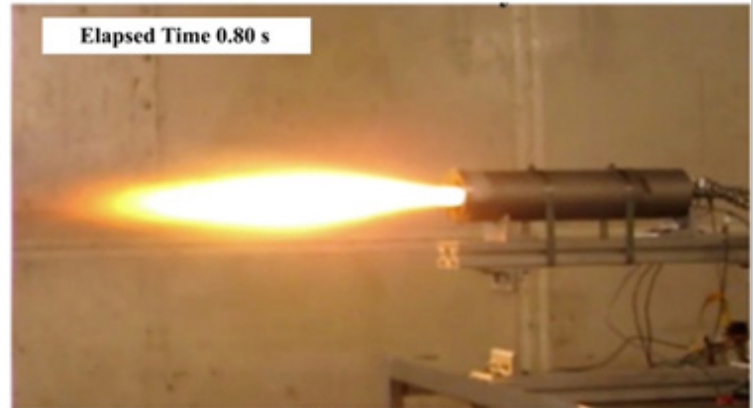

d) Fully Developed Hybrid Combustion

Figure 5 Still images from ignition development test with $120^{\circ}$ swirl, hollow-cone main injector.

This observed behavior is similar to behavior experienced by Rommingen \& Husdal $^{17}$ during testing of their lab-scale $87.5 \% \mathrm{H}_{2} \mathrm{O}_{2}$ $H T P B$ motor. Rommingen \& Husdal ${ }^{17}$ would experience ignition transients as long as two seconds where the propellant would initially "smolder" for as long as two seconds before building up sufficient chamber pressure and heat to allow full combustion. Nammo would refer to these two events as "monopropellant combustion mode" and "hybrid combustion mode." Because of the startup transients, where unburned peroxide floods the combustion chamber during the startup transient, the integrated specific impulse values are consistently lower than the instantaneous values.

For this test series the mean "steady" specific impulse $\left(I_{s p}\right)$ values, calculated as the time-average of all values that are within $95 \%$ of the peak thrust value, was approximately 215 seconds. The mean "cumulative" $I_{s p}$, calculated as a running summation of the total impulse and the total consumed propellant mass, was approximately 201 seconds. Because of the startup transients, where unburned peroxide floods the combustion chamber during the startup transient, the integrated specific impulse values are consistently lower than the instantaneous values. Tests were also performed by replacing the extruded ABS fuel sections with cast hydroxyl-terminated polybutadiene (HTPB) grain segments. Generally, the HTPB grains exhibited similar mean thrust land specific impulse levels, but were prone to erosive burning during burns exceeding 5 seconds duration. This erosive burning effect leads to significantly higher burn to burn thrust and total impulse variability.

\section{Catalyst augmentation of the hybrid motor arc- ignition system}

Clearly, the observed ignition transients, some as long as 2 seconds, are less than idea. This paper will explore several novel methods for coupling the catalyst bed with the arc-ignition technique in order to reduce the observed hybrid ignition latency. Because the catalyst bed does not have to act in a stand-alone capacity, as it would with a mono-propellant system; multiple low-cost alternatives to the traditional noble-metal catalyst metals will be explored. The objective is to discover materials and support mechanisms that will allow the catalyst bed to act as a pre-warmer so that at least partially decomposed and heater oxidizer will be injected into the combustion chamber. The goal is to significantly startup transient times, and allow an overall increase in ignition reliability and combustion efficiency. No catalyst heater will be used for this design.

\section{Background on catalyst materials for hydrogen peroxide systems}

At mass concentrations above $90 \%$ hydrogen peroxide is commonly referred to as high-test peroxide (HTP), and has been a well-known option in the monopropellant field for over half a century. ${ }^{18}$ Peroxide is a chemical compound consisting of two hydrogen atoms and two oxygen atoms held together with two hydrogen-oxygen bonds and one oxygen-oxygen bond. In its aqueous form, peroxide is a clear liquid with a specific gravity $10-40 \%$ higher than water. Hydrogen peroxide is capable of undergoing an energetic decomposition reaction. Figure 6 shows the associated end-to-end reaction, neglecting intermediate products. In this reaction both oxidation and reduction occur at the same time. With a $100 \%$ solution this reaction is produces up to 98.1 $k J(3.33 \mathrm{MJ} / \mathrm{kg})$ for every decomposed mole of peroxide. Typically, in an $85-90 \%$ aqueous solution, $\mathrm{H}_{2} \mathrm{O}_{2}$ is reasonably stable requiring an activation energy of approximately $75 \mathrm{~kJ} / \mathrm{mol}$ in the absence of a catalyst. ${ }^{19}$ 

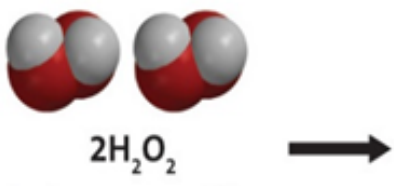

hydrogen peroxide

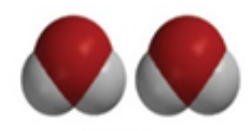

$2 \mathrm{H}_{2} \mathrm{O}$

water

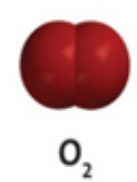

oxygen
Figure 6 Hydrogen peroxide decomposition reaction.

\section{Traditional noble metal catalyst materials}

In typical mono-propellant applications, the energy of activation $E_{a}$, can be significantly lowered using noble catalyst materials like silver, platinum, palladium, iridium, ruthenium. Catalyst beds made from silver or other noble metals can reduce the required activation from $75 \mathrm{~kJ} / \mathrm{mol}$ to less than $50 \mathrm{~kJ} / \mathrm{mol} .{ }^{18}$ Lowering the activation energy allows decomposition to begin with a significantly lowerenergy input. Figure 7 illustrates this activation energy concept. A study in 1963 by Runckel et al., ${ }^{20}$ outlined several silver-based catalyst bed builds for use with 98\% HTP. The authors achieved significant success with one particular configuration based on a piled series of silver-mesh screens. Following the publication of Runckel's report, activated-silver catbeds became the industry standard for high grade peroxide decomposition.

Unfortunately, there are several major drawbacks associated with using noble metals to build catalyst beds. These are;

a. Cost,

b. Weight,

c. Durability,

d. Break in and pre-conditioning,

e. Lack of effectiveness and decomposition efficiency.

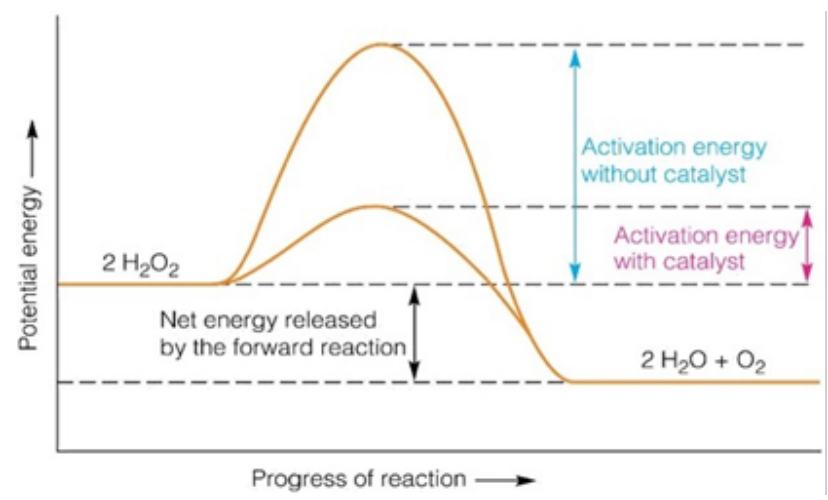

Figure 7 Hydrogen peroxide activation and decomposition energies.

The raw material required to build even a moderate massflow catalyst bed from noble metals can easily exceed one or more thousands of dollars. Because each catalyst bed is individually handcrafted and assembled in a one-off manner; manufacturing labor costs add significantly to the system cost. Catalyst beds fabricated from noble metals are extremely heavy and contribute nothing to the propulsive mass of the system.

Noble metal catalyst beds also have significant operational durability issues. Because the catalytic activity of the catalyst depends upon the available surface area indirect contact with the solution, oxidization due heating generally reduces the effectiveness of the catalyst bed. Similarly, stabilizers like tin salts, phosphates, nitrates, added to preserve the peroxide solution have the effect of reducing the catalyst surface area, and may in effect "poison" a working catbed rendering it ineffective. When working with higher concentrations of peroxide, the decomposition temperature can reach or even exceed the melting temperature of the noble-metal catalyst material. The result is a long term deforming, sintering, and potentially plugging the flow path. All these lead to a decrease in the performance and eventual failure of the catalyst bed. This lack of durability further adds to the costs of using silver or platinum in the catbed design.

Catalyst beds consisting of silver or platinum screens require a "break-in" period or some form of up front activation treatments. This initial break in period is not well understood and is only solved by running peroxide solution through the catalyst bed until full decomposition is achieved. The required "break-in" period can be quite significant. ${ }^{21}$ In order to reduce this this initial break-in period, catalyst materials are often subjected to a variety of activation processes. Two typical process involve soaking the base metal-screen materials in either nitric acid or samarium nitrate. Both of these activation methods are quite time consuming, and while nitric acid solutions are relatively benign; working with samarium nitrate poses major health concerns and must be handled with extreme caution. ${ }^{5}$ These recurring hands-on activities add to the cost of the catbed development.

Finally, there are simply better catalytic materials available than the traditional silver-screen designs. In an experimental characterization of a wide swath of catalytic materials has been carried out Rusek..$^{22}$ This author concluded that traditional silver-screen materials were, quite surprisingly, one of the least active catalyst materials. The least active catalysts were found to be samarium-promoted silver screens, i.e. the conventional peroxide decomposition catalysts. Thus, in light of the aforementioned drawbacks with silver catalysts, and considering that the desired objective is to develop a simple, light -weight, inexpensive catbed design that will act to "assist" the primary arc-ignition system; there are clearly a variety of options that can be reconsidered. The following subsection will identify well-known and existing material alternatives, and consider how those alternatives can be best adapted to meet the stated project objectives.

\section{Development of alternative catalysts for hydrogen peroxide decomposition}

Numerous research papers investigating the effectiveness of peroxide catalyst materials have been published. A significant number of authors including the previously discussed authors Runckel ${ }^{20}$ and Ponzo $^{21}$ have investigated the catalytic properties of silver and silver oxides. ${ }^{23-28} \mathrm{~A}$ similarly large group has investigated manganese dioxide $\left(\mathrm{MnO}_{2}\right)$ supported on alumina or titanium dioxide. ${ }^{29-35}$ Results are often conflicting. For example, Pirault-Roy et al., ${ }^{35}$ performed several tests on the activity of silver, platinum, and manganese dioxide and found that silver had the highest reactivity with hydrogen peroxide, followed by manganese dioxide, and finally platinum. Bramanti et al., ${ }^{36}$ also performed tests on these materials and showed that manganese (III) oxide $\left(\mathrm{Mn}_{2} \mathrm{O}_{3}\right)$ was the most reactive followed by silver and manganese dioxide. Metallic wires were also tested and showed that silver was the most reactive followed by platinum,

\footnotetext{
${ }^{5}$ Anon. Samarium Nitrate, Espi Metals.
} 
palladium, and then gold. An interesting observation by Bramanti ${ }^{36}$ was silver dissolving with time, thus limiting the available operational lifespan.

Previous research with noble metal-based catalysts: As mentioned earlier Runckel ${ }^{20}$ established the early prototype catalyst bed configuration using a pile of compressed silver mesh-screens. Of special interest for this work is Runckel's self-labeled "design \#2." In order to activate the screens, Runckel soaked the screens for several hours in a $10 \%$ samarium nitrate solution. The deposited samarium tends to act as a surfactant allowing the flowing peroxide streams to adhere to the silver-screen meshes longer. The layout consisted of a stainless steel distribution plate, followed by nearly 50, 20-mesh silver screens. Anti-channel baffles were also included. This design was the basis for the first hot-fire catalyst bed studied by this campaign and the precise details will be presented later in this paper. This design was generally effective for very high concentrations of peroxide at $98 \%$, but its performance dropped off as the peroxide concentration was reduced. This loss in efficiency has significant ramifications with regard to hybrid rocket applications and will be described later in this paper.

Jonker et al., ${ }^{23}$ set up the silver screen catbed using an initial injector plate. Following an injector plate, they inserted four inert mesh screens made of 304 stainless steel to initially diffuse the hydrogen peroxide flow. They followed the inert section with sections of 15, 40mesh screens of silver alternating with anti-channel baffles to prevent channeling flow from damaging the catalyst bed. Four additional inert screens of 304 stainless steel were added at the end before a support plate to give the catalyst bed added strength. To activate the silver, they were prepared in a $15 \%$ nitric acid solution and then heat treated the soaked screens at $900 \mathrm{~K}$. The post treated screens were tested and found to exhibit a much higher activity than the pre-treatment screens.

Cervone et al., ${ }^{25}$ used a base catalyst of 80 mesh silver interchanged with bigger meshes of silver and nickel for added support. Su-LiM $\&$ Choong-Won ${ }^{28}$ also explored silver screens as a catalyst. The silver screens were interspaced with other inert screens of stainless steel to add support and strength to the catalyst pack. A nitric acid solution was chosen to etch and activate the silver screens over the more complicated and hazardous samarium nitrate activation process. Several different configurations were built and tested. The catalyst beds were tested with $80 \%, 85 \%$, and $90 \%$ hydrogen peroxide. The combustion efficiency $\eta^{*}$ for each of the concentrations was above 90 $\%$ for each of the mass flow rates tested.

Other investigators including Ponzo $^{21}$ have tested alternative layouts for silver catalysts. Instead of the normal screen bed that is often used, a monolithic bed was developed. Consisting of stacked silver "platelets". These platelets were thin sheets of silver with varying sizes of holes on them. When stacked and bonded together, they offered high surface area contact with the peroxide. Ponzo reported that the bed delivers a lower pressure drop when compared to the traditional silver screen catalyst bed as well as improved lifespan. Using $90 \%$ concentrated peroxide and a massflow loading factor of $40 \mathrm{~g} / \mathrm{s}^{-\mathrm{cm}^{2}}$ over a wide range of feed pressures, the catalyst beds demonstrated good performance up to 900 total seconds of use. The break-in period for this catalyst bed was reported as less than 5 seconds in full flow. Unfortunately, this type of catalyst bed configuration is patented and licensing the design is cost prohibitive for this project.
Due to its reported exceptional reactivity Platinum as a catalyst was explored by An et al. ${ }^{26,27}$ An et al., ${ }^{26,27}$ used a precursor solution of $\mathrm{H}_{2} \mathrm{PtCl}_{6}$ to wet impregnate alumina pellets. The pellets were then dried and calcinated. Calcination is a process whereby a material is heated to a temperature slightly below the melting, causing loss of moisture, and deposition of any solids incorporated in the surface moisture. This process was carried out twice to obtain a high percentage coat of silver on the alumina pellets. Results showed that a decomposition efficiency of over $90 \%$ was achieved with the setup. A scaled-up thruster was also used with this type of catalyst bed. The authors did not discuss the effective lifetimes of the developed catalysts.

Previous development on alkali-metal based catalyst materials: Several readily available and inexpensive minerals including potassium permanganate $\left(\mathrm{KMnO}_{4}\right)$, sodium permanganate $\left(\mathrm{NaMnO}_{4}\right)$, and manganese dioxide $\left(\mathrm{MnO}_{2}\right)$ and are known to exhibit significant catalytic activity when immersed in peroxide solutions. Figure 8 shows samples of these minerals. Potassium permanganate, a common compound used for various applications in the medical and water treatment industries, is a strong soluble oxidizer. ${ }^{6}$ When exposed to hydrogen peroxide, the reaction forms diatomic oxygen, manganese dioxide, potassium hydroxide, and water. The decomposition reaction is

$$
3 \mathrm{H}_{2} \mathrm{O}_{2}+3 \mathrm{KMnO}_{4} \rightarrow 3 \mathrm{O}_{2}+2 \mathrm{MnO}_{2}+\mathrm{KOH}+2 \mathrm{H}_{2} \mathrm{O} \text { (1) }
$$

Although potassium is widely available, low-cost, non-toxic, and presents no objective hazards; as noted by Eq. (1), $\mathrm{KMnO}_{4}$ is not a true catalyst, but participates in the decomposition reaction. Thus, with prolonged use the catalyst will be consumed by the reaction. Sodium permanganate exhibits similar properties to potassium permanganate, except it is more expensive to produce.

Because both materials are water soluble they cannot be directly embedded into a catalyst bed. Instead, that the permanganate can be dissolved in solution and a porous supporting material used to absorb the solution. By heat treatment and washing, a form of $\mathrm{MnO}_{2} \mathrm{O}_{2}$ will be deposited onto the support. The resulting metal oxide is no longer water soluble, but is still highly catalytic in the presence of peroxide. While this process has been successfully carried out with sodium permanganate, ${ }^{35}$ the authors found no available literature describing this process being conducted with potassium permanganate. Jo et al., ${ }^{31,34}$ used sodium permanganate as a precursor solution for building up a manganese dioxide catalyst. Manganese dioxide ${ }^{7}$ is an inorganic solid that is mainly used in the production of dry-cell batteries. Manganese dioxide compound is not soluble in water, and should be more durable as a catbed material. designing possible catalyst bed solutions. Unlike $\mathrm{KMnO}_{4}, \mathrm{MnO}_{2}$ is a true catalyst of hydrogen peroxide and during the reaction the material is left unchanged, i.e. it is much less likely to be consumed and will exhibit a longer operational lifetime. Like potassium permanganate, manganese dioxide is easy to obtain, non-toxic, and low-cost. Its non-soluble characteristic helps the compound adhere to catalyst support better than a soluble one.

Jo et al. ${ }^{31}$ did extensive work with manganese dioxide supported on alumina pellets. Manganese dioxide is a black-to-brown solid that occurs naturally as the inexpensive mineral pyrolusite. Manganese oxide has a valence state of +4 and is quite reactive. A mixture of manganese dioxide and lead oxide was also tested and compared to

${ }^{6}$ Anon. Potassium Permanganate

${ }^{7}$ Anon. Manganese; manganese dioxide. WebElements; 2018. 
pure manganese dioxide. This $\mathrm{MnO}_{2} / \mathrm{PbO}$ helped reduce the chugging instability that was observed at low chamber pressures when only using a $\mathrm{MnO}$, catalyst. $\mathrm{An}^{30}$ and $\mathrm{Jo}^{31}$ report these results. Sorge et al., ${ }^{33}$ also used $\mathrm{MnO}_{2}$ but supported it on a titanium dioxide $\left(\mathrm{TiO}_{2}\right)$ pellet. These authors showed that the catalyst bed exhibited a very high level of initial activity that drops over time until a steady state value was reached. Maia et al., ${ }^{32}$ used a catalyst comprising of an extruded CoMnAl precipitate compound that showed no catalyst deactivation over time. The pellets showed no signs of degradation or fracturing and the bed itself suffered only minimal, but constant, pressure drops. One significant disadvantage of $\mathrm{MnO}$ is its relatively low melting temperature of $535^{\circ} \mathrm{C}$. When $\mathrm{MnO}$, melts, the compound decomposes and loses its $\mathrm{O}_{2}$ molecule. As shown later by Figure 11, the decomposition temperature of hydrogen peroxide is typically well above this melting point. Thus, it is highly likely that the catalyst will decompose during use. The authors could find no published literature detailing how rapidly the catalytic effectiveness of $\mathrm{MnO}_{2}$ dissipates during use.
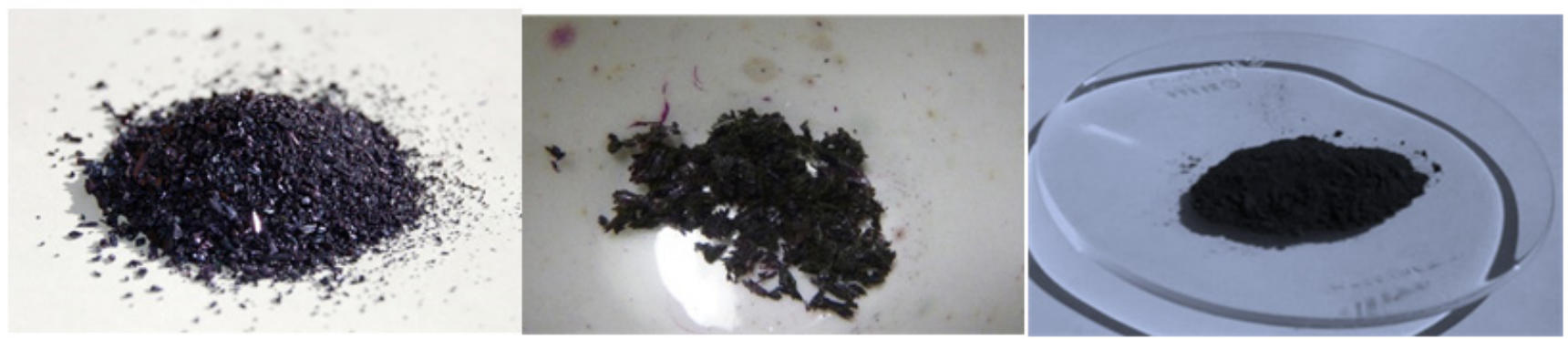

Figure 8 Left to right: potassium permanganate, sodium permanganate, and manganese dioxide.

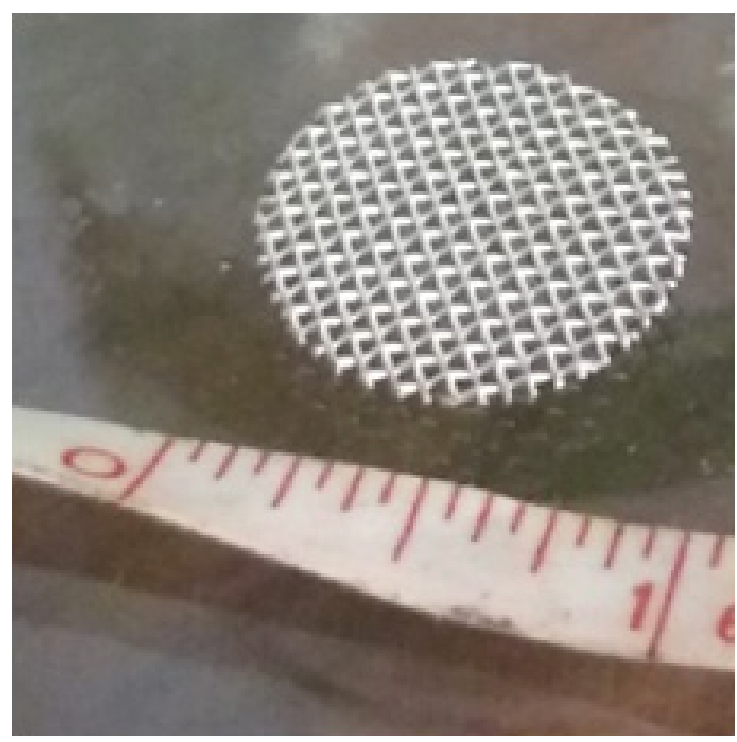

Figure 9 Example of a finished silver catalyst screen-round.
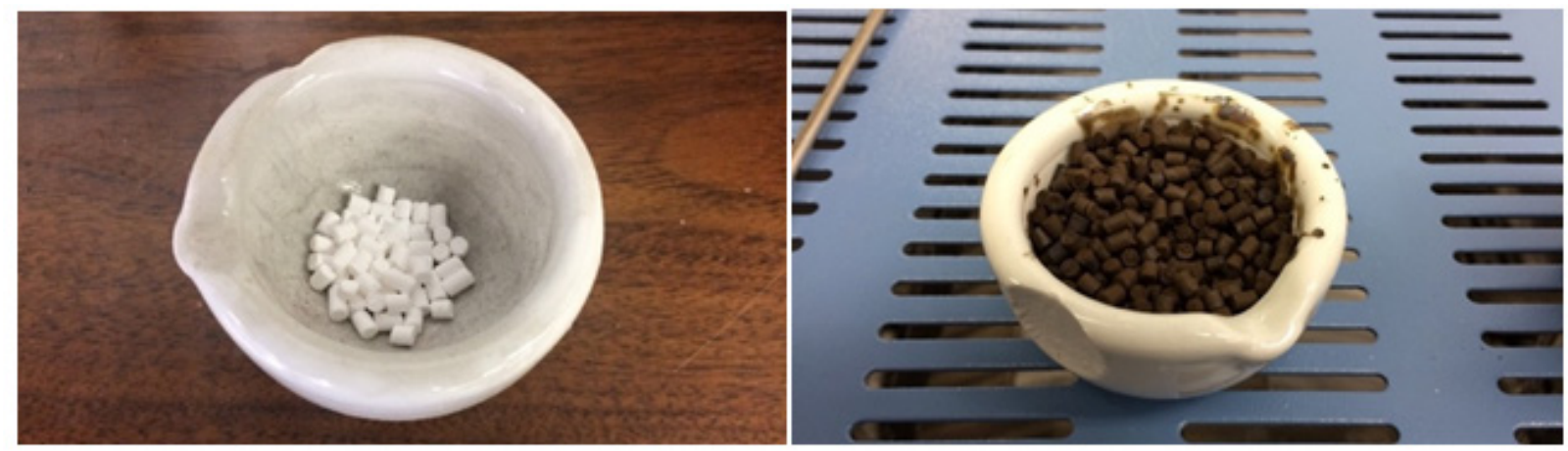

Figure 10 Plain $\gamma$-alumina pellets (left) and pellets with $\mathrm{MnO}_{2}$ deposited. 


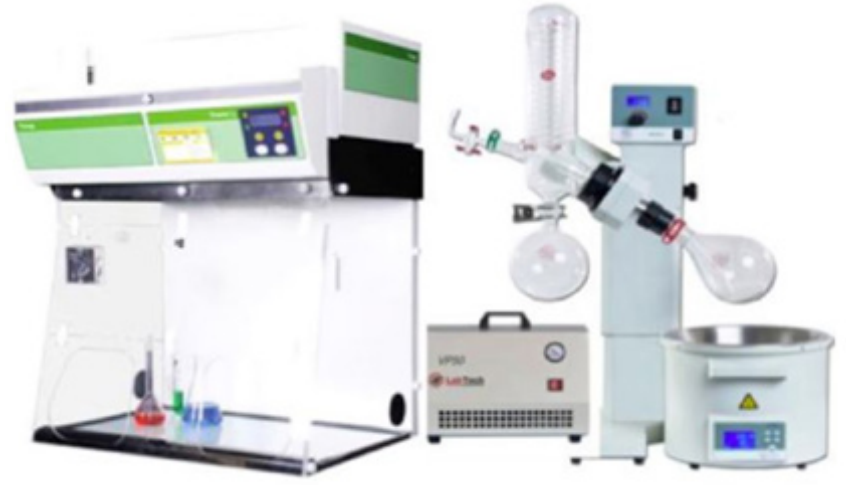

Figure II Rotary evaporator system used to condense high concentration peroxide from low-grade solution.

\section{Catalytic test material selection and preparation}

Based on the previously-described literature search-the catalytic materials silver and manganese dioxide-were down-selected for further investigation. The silver catbed, constructed as specified by Runcke $^{20}$ for the $\# 2$ configuration, was selected as the reference configuration. This effectiveness of this more-conventional design will be compared against an equivalently-sized catbed constructed using manganese dioxide deposited onto a ceramic-pellet support material. Specific details for the catbed designs and their interface with the hybrid motor systems will be described later in the paper.

\section{Silver-screen catalyst}

Because the Runckel ${ }^{20}$ silver-screen catbed was selected as the control design for the augmentation catalyst bed, the chosen form for the silver catalytic material was a commercially-available 20-mesh silver screen cloth $^{8}$ with a 0.016 in. $(0.0381 \mathrm{~cm})$ wire diameter. A series of 0.89 in. $(2.26 \mathrm{~cm})$ wire "rounds," were cut from this cloth. The diameters of the cut silver rounds, pictured by Figure 9, were calculated to allow the design oxidizer massflow for the hybrid motor, ${ }^{6}$ approximately $50 \mathrm{~g} / \mathrm{sec}$ with a cross-section massflux of approximately $12.5 \mathrm{~g} / \mathrm{cm}^{2}$-s. This massflux value was recommended by Bengtsson and Bengtsson ${ }^{9}$ based on extensive previous experience with monopropellant peroxide applications. Sample test specimens were treated by two mechanisms prominently suggested by the available literature, 1) soaking in a $10 \%$ solution of Samarium Nitrate, followed by heat treatment, ${ }^{18,20,37}$ and 2) soaking in a $30 \%$ nitric acid of various concentrations, followed by a post-soaking heat treatment. ${ }^{37,38}$

Estimating the contact surface index for the silver wire mesh: An important parameter that determines the effectiveness of a catalyst form factor is the surface contact index, CSI. For non-spherical shapes the CSI may be calculated as the surface area of a sphere containing a volume equivalent to the volume of the test specimen, $A s_{s p h}$. For homogeneous powders, Brahmanti et al., ${ }^{36}$ calculates the CSI by

$$
C S I=\frac{m}{\rho} \cdot \frac{A_{S_{s p h}}}{V_{p}}
$$

In Eq. (2) $m$ is the mass of material being used as the catalyst, $\rho$

\footnotetext{
${ }^{8}$ Anon. BWC, Wire Cloth, Wire mesh, and Woven Wire.

${ }^{9}$ Bengtsson E, and Bengtsson G. How do Hydrogen HTP Rockets Work? HTP Propulsion; 2010.
}

is the material density, and $V_{p}$ is the volume of the catalyst material. For a wire strand with a nearly cylindrical cross-section, the CSI may be estimated for the silver as spherical surface area in units of $\mathrm{mm}^{2}$,

$$
A_{S_{\text {sph }}}=\pi^{1 / 3}\left(6 \cdot V_{\text {wire }}\right)^{2 / 3}=\pi \cdot\left(\frac{3}{2} L_{\text {wire }} \cdot D_{\text {wire }}^{2}\right)^{2 / 3}
$$

In Eq. (3) $V_{\text {wire }}$ is the wire strand volume, $D_{\text {wire }}$ is the wire strand diameter, and $L_{\text {wire }}^{\text {wire }}$ is the effective strand length. For a wire screen with mesh number $M$, the projected empty area $W$ between wires is calculated as

$$
W=\frac{2.54_{c m}}{M}-D_{\text {wire }}
$$

The projected 2-D surface area of the wires $A_{\text {wire }}$ in a flat screen is calculated from the wire diameter and screen mesh number $M$ by

$$
\frac{A_{\text {wire }}}{A_{\text {screen }}}=1-\left(\frac{W}{W+D_{\text {wire }}}\right)^{2}=\frac{\left(2 \cdot W+D_{\text {wire }}\right) \cdot D_{\text {wire }}}{\left(W+D_{\text {wire }}\right)^{2}}
$$

In Eq. (5), $A_{\text {screen }}$ is the total screen element 2-D surface area of a circular screen segment. The wire screen area is related to the wire diameter and effective length by

$$
A_{\text {wire }}=\frac{\pi}{4}\left(L_{\text {wire }} \cdot D_{\text {wire }}\right)
$$

and the effective wire length is

$$
\begin{aligned}
& L_{\text {wire }}=\frac{4}{\pi} \cdot \frac{A_{\text {wire }}}{D_{\text {wire }}}=\frac{4}{\pi} \cdot \frac{1}{D_{\text {wire }}} \frac{\left(2 \cdot W+D_{\text {wire }}\right) \cdot D_{\text {wire }}}{\left(W+D_{\text {wire }}\right) 2} . \\
& A_{\text {Screen }}=\frac{4}{\pi} \cdot \frac{\left(2 \cdot W+D_{\text {wire }}\right)}{\left(W+D_{\text {wire }}\right) 2} \cdot A_{\text {Screen }}
\end{aligned}
$$

\section{Manganese dioxide catalyst}

As recommended by Jo et al., ${ }^{31}$ the manganese was prepared as a catalyst by depositing the material onto $1 / 8 \mathrm{in}$. diameter by $1 / 4 \mathrm{in}$. length $\gamma$-alumina pellets acting as support material. ${ }^{10}$ The $\gamma$-alumina pellets were wet impregnated by soaking for 8 hours at ambient temperature in a $50 \%$ mass concentration podium permanganate solution. The pellets were left to soak at ambient temperature for 8 hours. After soaking, they were dried at $200^{\circ} \mathrm{C}$ for 15 hours and then calcined at $800^{\circ} \mathrm{C}$ for 2 hours using a high-temperature tube furnace. ${ }^{11}$ Figure 10 shows the pellets before and after $\mathrm{MnO}_{2}$ deposition. Each treated pellet was counted and the collected set of pellets was weighed before and after in order to calculate the mean mass of catalyst deposited onto each pellet.

Estimating the contact surface index for the silver wire mesh: For each coated pellet the CSI is calculated as

$$
\text { CSI }=\frac{m}{\rho} \cdot \frac{A_{S_{s s p h}}}{V_{p}} \approx \pi^{1 / 3}\left(6 \cdot \pi \cdot D_{\text {pellet }}^{2} \cdot L_{\text {pellet }}\right)^{2 / 3}
$$

\footnotetext{
${ }^{10}$ Alfa Aesar, 43832 Aluminum oxide, gamma-phase, catalyst support, high surface area, bimodal.

${ }^{11}$ Anon. Mini CVD Tube Furnace with 2 Channel Gas Mixer, Vacuum Pump, and Vacuum Gauge - OTF-1200X-S50-2F.
} 
In Eq. (8) $D_{\text {pellet }}$ is the approximate diameter of each coated pellet, and $L_{\text {pellet }}$ is the effective length. The effective lengths and diameters also includes twice the thickness of $\mathrm{MnO}_{2}$ deposited onto the alumina surface,

$$
\begin{aligned}
& D_{\text {pellet }}=D_{\text {almunia }}+2 \cdot \sqrt[3]{\left(\frac{m}{\rho}\right)_{M n O_{2}}} \\
& L_{\text {pellet }}=L_{\text {almunia }}+\cdot \sqrt{\left(\frac{m}{\rho}\right)_{M n O}}
\end{aligned}
$$

\section{Distillation of high purity, high concentration hydrogen peroxide}

One of the more significant challenges associated with this research campaign was procuring high-purity peroxide solutions at a concentration level that was useful for our propulsion applications, between 85 and $90 \%$. Nearly all commercially available peroxide at high concentration levels contains some form of stabilization in order to make the solutions long-term storable. Common stabilizers include chelants, tin and sodium pyrophosphate, and sequestrants, colloidal silicate. ${ }^{12}$ These additives are known to effectively poison catalytic materials, especially silver; and the authors believed that this effect would bias the test comparisons. As an added benefit, using this approach means that large quantities of high-grade peroxide need not be stored on site. This advantage leads to considerable infrastructure and operational cost savings for this test campaign. Thus, as an Operating Principle, the project decided it would only keep on hand the amount of high concentration peroxide solution necessary to complete a single day's-worth of testing, with maximum allowable storage time of two days. Only $50 \%$ or lower concentrations of $\mathrm{H}_{2} \mathrm{O}_{2}$ were stored for more than two days.

To solve the described problem, the authors have developed a condensation procedure to manufacture small amounts of high purity, high concentration. The method was adapted from an earlier procedure established Rarata \& Surmacz. ${ }^{37}$ In this approach a lowgrade peroxide solution is condensed to high concentration using a custom built distillation apparatus. Figure 11 shows the evaporator arrangement with the laboratory-quality Wilmad WG-EV311 rotary evaporator ${ }^{13}$ installed under a fume hood to collect any extraneous peroxide vapor. In this procedure a sample flask containing the low concentration peroxide solution rotates in the temperature controlled bath. A vacuum pump attaches to top of condensing chamber to lower the evaporation point of the solution. An isolated coiled tube runs through condensing chamber. Ice water is pumped through the coils to condense the evaporated fluid. Low peroxide concentration condensate collects in flask at bottom of condensing chamber. The remaining material in the distillation flask grows increasingly more concentrated with time.

The vacuum level, bath temperature, and rotation speed are adjustable. The current process uses a regulated $55^{\circ} \mathrm{C}$ heater bath and a soft vacuum of approximately 0.1 atmospheres. The low $55^{\circ} \mathrm{C}$ bath temperature minimizes the peroxide loss to the distillate and ensures that thermal decomposition will not occur during distillation.

\footnotetext{
${ }^{12}$ Anon. What are the stabilizers of Hydrogen Peroxide.

${ }^{13}$ Anon. WG-EV311 Rotary Evaoporator, Vertical Condenser (110/115V Only), Wilmad-Labglass, SP Scienceware.
}

A closed-cycle greaseless pump is used to draw the vacuum. With this setup the team was able to reduce $50 \%$ grade peroxide to $75-85 \%$ concentration, producing as much as $400 \mathrm{ml}$ of concentrated solution within $4-5$ hours. The process loses approximately $50 \%$ of the original volume, and is thus about $80 \%$ efficient in terms of peroxide retention. In this one-step process the vacuum and temperature setting are such that the water is solution is vaporized, but the peroxide and stabilizers remains behind. Two readily-available sources of low-grade peroxide were evaluated for this project. Originally, all condensation operations were performed using $50 \%$ concentration product available from Sigma-Aldrich. ${ }^{14}$ It was discovered that this product contains a proprietary stabilizer formula with tin compounds and chelating agents. A second and desirable product was supplied by Thermofisher Scientific ${ }^{15}$ in a $30 \%$ concentration. At this concentration level, there is no need for added stabilizers, and the product is certified by the American Chemical Society as reagent grade purity.

Using the Sigma-Aldrich $50 \%$ product as the starting solution, concentrating to greater than $85 \%$ was problematic due to the precipitation of stabilizers and solution "clouding". The clouding results from the stabilizers being concentrated in the condensed solution. Using the Sigma-Aldrich $50 \%$ product it is likely that a double distillation process will be required to achieve a $90 \%$ or greater concentration. In contrast the ThermoFisher $30 \%$ product did not use stabilizers and the resulting condensates remained clear regardless of the final concentration. Interestingly, once the laboratory switched to the $30 \%$ ThermoFisher Scientific product, then the time required to reduce to a $90 \%$ concentration dropped considerably, to approximately three hours. Also, the resulting end-product did not exhibit the visual cloudiness observed for the solution derived from derived from the 50\% Sigma-Aldrich product. Apparently, the stabilizers in the $50 \%$ solution also served to make it considerably more difficult to condense to high concentration.

\section{Augmentation catalyst bed design, modeling, and installation}

The augmentation catalyst bed was not a "clean sheet design," but instead musty be adapted to fit the existing test article described earlier in Section III. In order to adapt the system for $\mathrm{H}_{2} \mathrm{O}_{2}$ as the oxidizer, a catalyst holder made from an industrial-grade sanitary fitting was installed into the peroxide feed line upstream of the motor. The catalytic materials are housed within the sanitary fitting. Decomposition output products are funneled into the motor combustion chamber through the normal oxidizer flow path. An external band heater was used to pre-heat the catalyst bed before peroxide flow was initiated. Figure 12 shows the overall systems concept, where the peroxide is fed into the thruster chamber, and the resulting decomposition products are blended with gaseous oxygen just before injection into the thrust chamber. Figure 3 shows the motor cap with the peroxide and GOX ports depicted. The interior of the catbed holder is sufficiently long to allow for a catalyst of up to $8.25 \mathrm{~cm}$ (3.25 in.) long, and has an internal diameter of $2.21 \mathrm{~cm}(0.89 \mathrm{in}$.). The maximum available $\mathrm{L} / \mathrm{D}$ or the catbed is correspondingly 3.735 . The entire assembled catbed fixture weighs approximately 620 grams.

${ }^{14}$ Sigma-Aldrich. Safety Data Sheet, Hydrogen Peroxide Solution, Product No. 516813,50 wt. $\%$ in $\mathrm{H}_{2} \mathrm{O}$, Stabilized.

${ }^{15}$ ThermoFisher Scientific. Safety Data Sheet, Hydrogen Peroxide, 30\%, Cat. No. H325-4; 2007. 


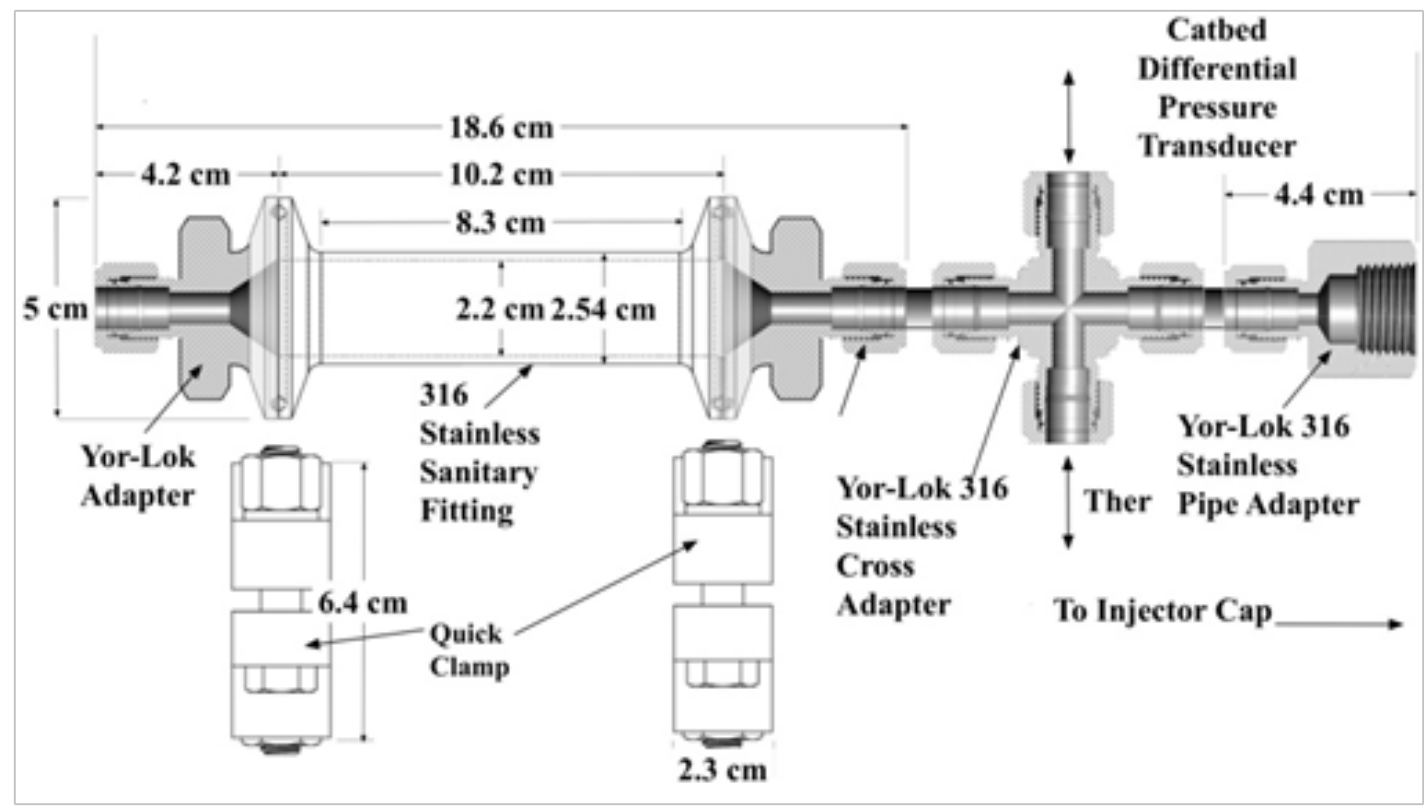

Figure 12 Catalyst bed holder installed upstream of the hybrid motor combustion chamber injection section.

\section{“Runckel” \#2 silver-screen catalyst bed}

As mentioned Runckel \#2 silver screen catbed design, ${ }^{20}$ was used as the baseline/control for this test series. Figure 13 depicts this catbed layout. As described in the previous section, the $0.89 \mathrm{in}$. screen diameter was selected to allow the original design massflow of $50 \mathrm{~g} / \mathrm{s}$ with a massflux of approximately $12.5 \mathrm{~g} / \mathrm{cm}^{2}-\mathrm{s}$. The distribution plates direct the flow into the core of the catalyst bed. Runckel recommends that the total area of the distribution ports be approximately equal to one third the total cross sectional area. Working within those recommendations, 14 holes with $1 / 8$ " diameters were drilled in the plates. The anti-channel baffles prevent non-decomposed peroxide from streaming along the tube walls at the edges of the catalyst bed screens. The baffles are 0.25 " thick stainless steel pipe with an outer diameter of 0.87 " and an inner diameter of 0.805 ". The stainless steel and monel screens are added in order to increase the strength of the stack. The design consists of the following elements in order,
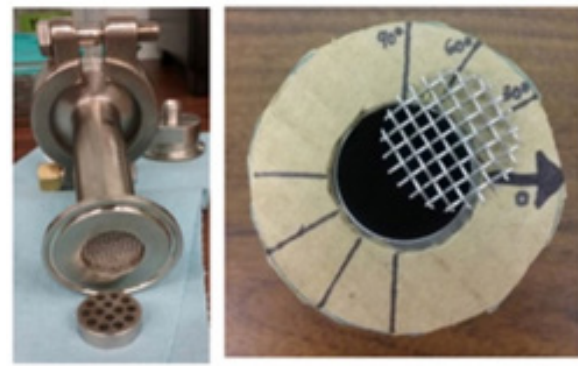

\section{a) Partially Assembled Catbed with Distributuon Plate and Screen Rotation Guide}

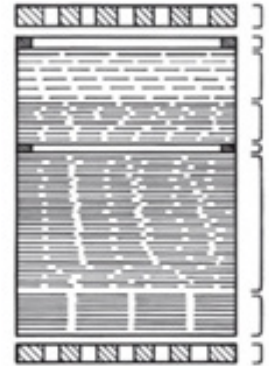

b) Runkel \#2 CatBed Screen Layout
Figure I 3 Runckel \#2 catbed design details.

a. 1, 0.87" diameter, 0.25 " thick, 316 stainless-steel distribution plate b. 2, 20 mesh $0.016,316$ stainless screens

c. 1, 0.87" diameter, $0.25 "$ thick, 316 stainless-steel anti-channel baffle

d. 20,20 mesh, 0.016 silver screens

e. $1, .87$ " diameter, 0.25 " thick 316 stainless-steel anti-channel baffle

f. 40, 20 mesh, 0.016 silver screens

g. 2,24 mesh, 0.016 nickel alloy screens

h. 13, 10 mesh, 0.025 nickel alloy screens

i. $1,0.87$ " diameter, 0.25 " thick 316 stainless-steel distribution plate

All of the components were packed into a 1" pipe sanitary fitting with each screen rotated at 30 degrees before being inserted using the guide to help rotational positioning. Per Runckel's design guide, the entire pack was compressed to approximately 2,200 psi. Compressing the pack decreased the "pile" by 1.25 " for a final length of silver screens of approximately 2.42 " $(6.15 \mathrm{~cm})$. The compressed catalyst stack weighed approximately 95 grams. A spacer was used to secure the catbed inside of the 3.2" long support fitting.

\section{$\mathrm{MnO}_{2}$ catalyst bed design}

The pellet bed design follows recommendations offered by $\mathrm{Jo}^{31}$ \& Salahudden ${ }^{39}$. The same 0.89 " inner diameter sanitary fitting housing was used for the pellet-based catbed. Figure 14 shows the layout. The configuration consists of the same distribution plates used to direct the flow into the bed. After the distribution plates, two monel screens were used to partially atomize the incoming flow and contain the pellets. Then flow then passes through the core of the pellet bed. Two more Monel screens were added and finally the exit support plate. Approximately 20 grams of prepared catalyst pellets could fit into the catbed housing without compression. 


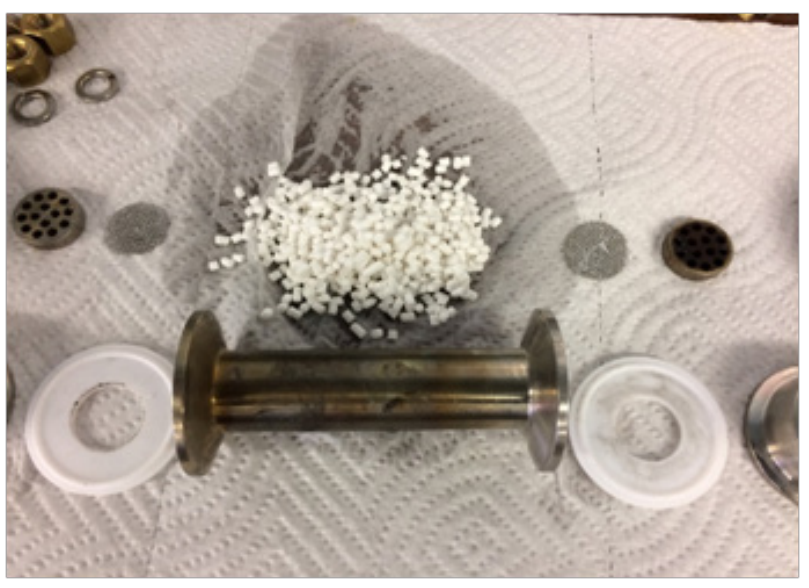

Figure 14 Layout of pellet bed.

\section{Catbed design efficiency considerations}

Reduced efficiency of the catbed has a critical effect on lowering the peroxide decomposition temperature. Thus, choosing an appropriate catbed geometry is critical in order to avoid a "wet start" that may affect ignitability of the system. As previously presented, the fundamental decomposition reaction for $n$ moles of pure $\mathrm{H}_{2} \mathrm{O}_{2}$ is

$$
n_{\mathrm{H}_{2} \mathrm{O}_{2}}=\frac{1}{2} n_{\mathrm{O}_{2}}+n_{\mathrm{H}_{2} \mathrm{O}}
$$

If only a fraction of the peroxide mass decomposes, then the fundamental reaction for pure peroxide is

$$
n_{\mathrm{H}_{2} \mathrm{O}_{2}}=\eta \cdot\left(\frac{1}{2} n_{\mathrm{O}_{2}}+n_{\mathrm{H}_{2} \mathrm{O}}\right)+(1-\eta) n_{\mathrm{H}_{2} \mathrm{O}_{2}}
$$

In Eq. (11) $\eta$ is the decomposition efficiency, and the $M_{w}$ 's are the approximate molecular weights of pure peroxide $\left(34.01 \mathrm{~kg}_{\mathrm{kg}-\mathrm{mol}}\right)$, and water $\left(18.015_{\mathrm{kg} / \mathrm{kg}-\mathrm{mol}}\right)$. Defining the concentration mass ratio as

$$
f=\frac{m_{\mathrm{H}_{2} \mathrm{O}_{2}}}{m_{\mathrm{H}_{2} \mathrm{O}}+m_{\mathrm{H}_{2} \mathrm{O}_{2}}}
$$

substituting into Eq. (11) and ignoring the heat capacity of the undecomposed peroxide gives the "effective" mass ratio $f$ for energy release for an inefficient decomposition reaction

$$
f^{\prime}=\frac{\eta}{[1-f \cdot(1-\eta)]} \cdot f
$$

Figure 15 plots the predicted decomposition temperatures as a function of decomposition efficiency and HTP concentration. For example, reading Figure 15, a 90\% peroxide solution with $\eta=50 \%$ decomposition efficiency gives an effective mass fraction of $81.8 \%$. Thus, the corresponding exhaust temperature will be reduced accordingly from approximately $740^{\circ} \mathrm{C}$ to $550^{\circ} \mathrm{C}$. This inefficiency and resulting reduced temperature will have critical effects with regard to hybrid rocket operation. During the initial hybrid rocket ignition sequence the combustor chamber pressure lies at or near the ambient operating conditions. When the partially-decomposed, relatively high-pressure peroxide stream leaves the catbed and enters the initially low-pressure combustion chamber; the expansion will cause significant adiabatic cooling to occur. The data plotted on Figure 16 reinforce this assertion. Here the plume temperatures entering the combustion chamber are calculated using the NASA equilibrium Chemistry Program CEA, ${ }^{40}$ at various $P_{c} / P_{\text {exit }}$ ratios. Plotted are the predicted initial combustion chamber temperatures for $87.5 \%$ peroxide decomposition at $\{100 \%, 90 \% 80 \%, 70 \%, 60 \%$, and $50 \%$ decomposition efficiencies. Also plotted are typical pyrolysis temperatures for the hybrid previously-described rocket fuels ABS $\left(\sim 380^{\circ} \mathrm{C}\right)^{41}$ and $\mathrm{HTPB}\left(\sim 435^{\circ} \mathrm{C}\right) . .^{42}$

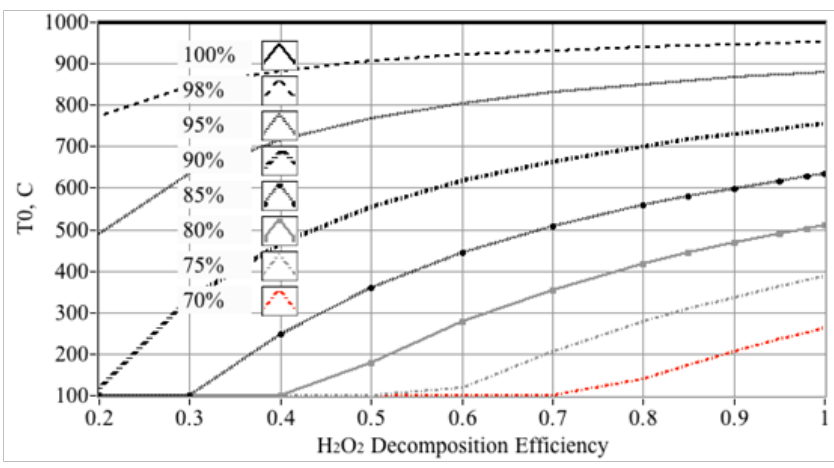

Figure 15 Estimated exit temperature as a function of catalyst bed decomposition efficiency and peroxide mass concentration.

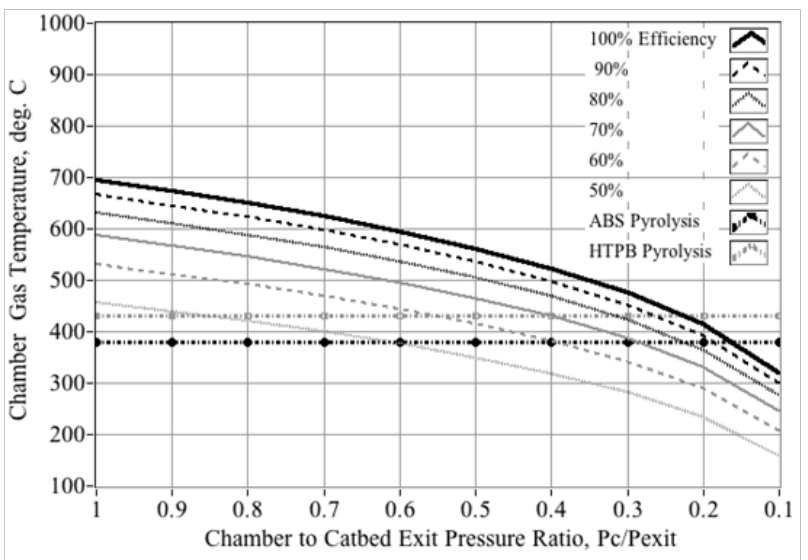

Figure 16 Effect of expansion ratio and combustion efficiency on precombustion chamber temperatures.

Note, that even at $100 \%$ decomposition efficiency, the chamber gas temperature does not exceed the pyrolysis temperature of HTPB unless $P_{c} / P_{\text {exit }}$ exceeds approximately 0.2 . For ABS which pyrolyzes at a lower temperature, that value drops to 0.15 . As an example, assuming a catbed exit pressure of 250 psia, then under steady flow conditions and perfect decomposition efficiency, fuel pyrolysis will not begin until the chamber pressure reaches at least 50 psia for HTPB and 35 psia for ABS. Below these chamber pressure ratios, the cooled gaseous by products enter the chamber at temperatures substantially below the pyrolysis temperatures of the fuel. Without fuel pyrolysis, then complete combustion will not occur. If pressure drop $P_{c} / P_{\text {exit }}$ is sufficiently large, then the entering peroxide plume will supercool; forcing even fully vaporized water to condense into liquid form. Such "soaked" fuel grain is extremely difficult to ignite. 


\section{Catbed design model}

The semi-empirical catbed design model to be presented here is based on work originally presented by Folger \& Scott ${ }^{49}$ The method has been modified here to account for the cylindrical catalyst bed configuration. The approach assumes a constant total massflow rate through the catbed, and a constant, homogenous internal flow porosity.

Rate of reaction: The model starts with the Arrhenius equation which is used to predict the effect of the temperature rise along the length of the catbed $L$ upon the local rate of reaction,

$$
k_{r}=A \cdot e^{-\frac{\Delta E_{a}}{R_{u} \cdot T}}
$$

In Eq. (14) $R_{u}$ is the universal gas constant, $T$ is the mean reaction temperature, expressed in Kelvins, $\Delta E_{a}$ is the activation energy of the reaction, and $A$ is the scale or frequency factor for the reaction rate. Assuming a simple first order reaction of the form described by Figure 6 , then the molar rate of change of the peroxide solution is

$$
\dot{\mathscr{X}}=-k_{r} \cdot \mathscr{K}
$$

In eq. (15) $\mathrm{M}$ is the instantaneous solution molarity given by the number of moles of solute $\left(\mathrm{H}_{2} \mathrm{O}_{2}\right)$ per liter of solution. The molar decomposition rate changes along the length of the catalyst bed according to

$$
\frac{\partial \dot{n}}{\partial V}=\frac{\partial \dot{n}}{\phi \cdot A_{c} \partial L}=-k_{r} \cdot \mathscr{K}
$$

In Eq. (16) the parameters $V, \phi, A c$, and $L$ are the catbed internal volume, flow porosity, geometric cross section, and catbed flow path length.

Peroxide massflow concentration: Integrating Eq. (16) along the length of the catbed

$$
\dot{n}(L)_{\mathrm{H}_{2} \mathrm{O}_{2}}=\dot{n}(L)_{\mathrm{H}_{2} \mathrm{O}_{2}}-\phi \cdot A c \cdot \int_{0}^{L} k_{r} \cdot \mathscr{K} \cdot d s
$$

Equation (17) is converted to peroxide massflow rate by multiplying through by the molecular weight of peroxide

$$
\dot{m}(0)_{\mathrm{H}_{2} \mathrm{O}_{2}}=\dot{m}(L)_{\mathrm{H}_{2} \mathrm{O}_{2}}-M w_{\mathrm{H}_{2} \mathrm{O}_{2}} \cdot \phi \cdot A c \cdot \int_{0}^{L} k_{r} \cdot \mathscr{K} \cdot d s
$$

the peroxide massflow rate is related to the total massflow rate by

$$
\dot{m}_{\mathrm{H}_{2} \mathrm{O}_{2}}=f \cdot \dot{m}
$$

Also, the solution molarity is related to the mass concentration by

$$
\begin{aligned}
& \boldsymbol{M}=\frac{n_{\mathrm{H}_{2} \mathrm{O}_{2}}}{V_{\text {liters }}}=\frac{m_{\mathrm{H}_{2} \mathrm{O}_{2}}}{M_{w_{\mathrm{H}_{2} \mathrm{O}_{2}}}} \cdot \frac{1}{V_{\text {liters }}}= \\
& f\left(\frac{m_{\mathrm{H}_{2} \mathrm{O}}+m_{\mathrm{H}_{2} \mathrm{O}_{2}}}{V_{\text {liters }}}\right) \cdot \frac{1}{M_{w_{\mathrm{H}_{2} \mathrm{O}_{2}}}}=1000 \cdot f \cdot \frac{\rho_{\text {so } \mathrm{ln}}}{M_{w_{\mathrm{H}_{2} \mathrm{O}_{2}}}}
\end{aligned}
$$

Substituting Eqs. (20) and (19) into Eq. (18) gives,

$$
f_{(\mathrm{L})} \cdot \dot{m}=f_{(0)} \cdot \dot{m}-M w_{\mathrm{H}_{2} \mathrm{O}_{2}} \cdot \phi \cdot A c \cdot \int_{0}^{L} k_{r} \cdot 1000 \cdot f \cdot \frac{\rho_{\text {so } \mathrm{ln}}}{M_{w_{\mathrm{H}_{2} \mathrm{O}_{2}}}} \cdot d s
$$

Assuming steady massflow throughout the catbed

$$
f_{(\mathrm{L})}=f_{(0)}-1000 \frac{\phi \cdot A c \cdot \int^{L} k_{r} \cdot f \cdot \rho_{s o \ln } \cdot d s}{\dot{m}}
$$

Enthalpy of combustion and temperature change across catbed: The temperature distribution along the length of the catbed is described by the 1-dimensonal energy balance equation, where

$\dot{m} \cdot C_{\mathrm{p}(\mathrm{L})} \cdot T_{(\mathrm{L})}=\dot{m} \cdot C_{\mathrm{p}(0)} \cdot T_{(0)}+M w_{\mathrm{H}_{2} \mathrm{O}_{2}} \phi \cdot A c \cdot \int_{0}^{L} \Delta H_{d} \cdot k_{r} \cdot \mathscr{K} \cdot d s$

Using Eq. (21) and (22) to write in terms of mass fraction, Eq. (23) reduces to

$$
C_{\mathrm{p}(\mathrm{L})} \cdot T_{(\mathrm{L})}=C_{\mathrm{p}(0)} \cdot T_{(0)}+1000 \frac{\phi \cdot A c \cdot \int_{0}^{L} \Delta H_{d} \cdot k_{r} \cdot f \cdot \rho_{s o \ln } \cdot d s}{\dot{m}}
$$

In Eq. (24) $C_{p}$ is the specific heat of the solution, $\Delta H_{d}$ is the enthalpy of decomposition, and $\mathrm{T}$ is the local cross section temperature within the catbed. The other parameters have been previously defined.

The local enthalpy of decomposition is strongly a function of the local solution mass fraction. Although, it is well known that the total decomposition of pure hydrogen peroxide produces up to $98.1 \mathrm{~kJ}(3.33$ $\mathrm{MJ} / \mathrm{kg}$ ), rapid catalytic decomposition often leaves residual peroxide that is not decomposed. The total decomposition energy is reduced by the heat capacity and enthalpy of vaporization of the residual peroxide. For aqueous solutions, the heat capacity and vaporization energy of the solvent (water) also further reduce the output energy. Figure 17 plots the enthalpy of decomposition for aqueous peroxide solutions calculated using these loss considerations. This data was derived from Schumb et al. ${ }^{43}$ This figure also plots the enthalpy of decomposition of pure peroxide scaled by the solution mass fraction. Note that the actual decomposition energy, depending on the mass concentration, consistently runs approximately $0-10 \%$ lower than the theoretical value.

Catbed pressure loss: The pressure drop across the catalyst bed, with the decomposing fluid and variable longitudinal properties is extremely complex and impossible to calculate using first-principal considerations. However, the semi-empirical Ergun equation ${ }^{44,45}$ for packed pebble beds has been previously applied with some success to reacting flows, ${ }^{46}$ and offers a reasonable starting point for this calculation.

When applied to the geometry of the problem the Ergun equation reduces to 
$P_{(\mathrm{L})}=\mathrm{P}_{(0)}-\int_{0}^{L}\left(\frac{\dot{m} / A_{c}}{\rho_{s o \mathrm{ln}} \cdot d_{p}} \cdot \frac{1-\phi}{\phi^{3}}\right)\left(\frac{150 \cdot \mu \cdot(1-\phi)}{d_{p}}+1.75 \cdot\left(\dot{m} / A_{c}\right)\right) \cdot d s$

In Eq. (25) $A_{c}$ is the geometric cross section of the catbed flow path, $\mu$ is the solution viscosity, and $d_{p}$ is the equivalent particle diameter of the packed bed, approximated by

$$
d_{p}=\Phi \cdot D_{\text {wire }}
$$

In Eq. (26) the equivalent particle diameter is calculated based on the effective "sphericity," $\phi$ a measure of is the measure of how closely the shape of an object approaches that of a mathematically perfect sphere.

$$
\begin{aligned}
& \text { sphericity } \rightarrow \Phi_{\text {mesh }}=\frac{\text { surface area of sphere of same volume as particle }}{\text { surface area of particle }} \\
& =\pi^{1 / 3} \frac{\left(6 \cdot V_{p}\right)^{2 / 3}}{A_{p}} \\
& \Phi_{\text {mesh }}=\pi^{1 / 3} \frac{\left(6 \cdot \pi \cdot L \cdot D_{\text {mesh }}^{2} / 4\right)^{2 / 3}}{\pi \cdot L \cdot D_{\text {mesh }}}=\left(\frac{3}{2}\right)^{2 / 3} \frac{\left(L \cdot D_{\text {mesh }}^{2}\right)^{2 / 3}}{L \cdot D_{\text {mesh }}} \\
& =\left(\frac{3}{2}\right)^{2 / 3} \frac{D_{\text {mesh }}{ }^{1 / 3}}{L^{1 / 3}}=\left(\frac{3}{2}\right)^{2 / 3}\left(\frac{D_{\text {mesh }}}{L}\right)^{1 / 3}
\end{aligned}
$$

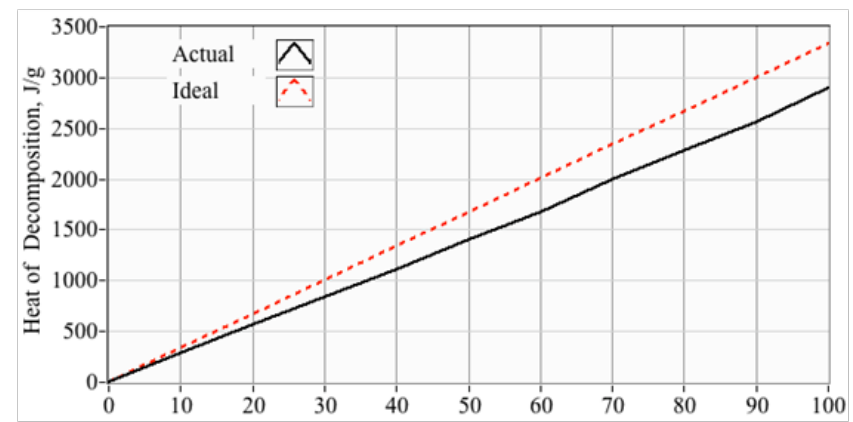

Figure 17 Enthalpy of decomposition of aqueous peroxide solutions.

Collected difference equations: The temperature at each point is calculated from the combined enthalpy rate and the molar flow rates of the species. The enthalpy of mixing is assumed to be zero for this simplify the model. Currently, water is assumed to vaporize at the boiling point of water, and the hydrogen peroxide is left in liquid phase until it is consumed. The density of each species is assumed to be independent of the others and the total density is a volume averaged density. Eqs (22), (24) and (26) are numerically integrated across the catbed length by diving into small elements with length $\Delta x$ and using a forward integration scheme. The collected solution equations are

$$
\begin{aligned}
& \text { Reaction rate }: \mathrm{k}_{\mathrm{r}}=\mathrm{A} \cdot \mathrm{e}^{-\frac{\Delta E_{a}}{R_{u} \cdot T}} \\
& \text { Mass Concentration }: f_{j+1}=f_{j}-1000 \frac{\phi \cdot A_{c} \cdot\left(\mathrm{k}_{\mathrm{r}} \cdot f \cdot \rho_{s o l \mathrm{ln}}\right)_{j}}{\dot{m}} \Delta x
\end{aligned}
$$

$$
\begin{gathered}
\text { Temperature }: T_{j+1}=T_{j}+\Delta H_{r} \cdot\left(1000 \frac{\phi \cdot A_{c} \cdot\left(\mathrm{k}_{\mathrm{r}} \cdot f \cdot \rho_{s o \ln }\right)_{j}}{C_{p_{j}} \dot{m}} \Delta x\right) \\
\text { Pressure Loss }: P_{j+1}=P_{j}-\left(\frac{\dot{m} / A_{c}}{\left(\rho_{\text {soln }}\right)_{j} \cdot d_{p}} \cdot \frac{1-\phi}{\phi^{3}}\right) \\
\left(\frac{150 \cdot \mu_{j} \cdot(1-\phi)}{d_{p}}+1.75 \cdot\left(\dot{m} / A_{c}\right)\right) \cdot \Delta x
\end{gathered}
$$

In Eq. (28) the solution properties $\rho_{\text {soln }}, C_{p}$, and $\mu$ are calculated as a function of temperature and mass concentration from lookup tables presented by Ebelke. ${ }^{47}$

\section{Catalytic activity tests}

Although both Rusek ${ }^{22}$ and Brahmanti et al., ${ }^{36}$ performed extensive studies to assess the relative catalycity of both silver and manganese dioxide, both of these studies used the materials in powdered form and not in the form that will be used to build the ignition augmentation catbeds. For this reason, it was decided to perform a closely-controlled set of baseline reactivity tests using both materials before integrating them into the ignition augmentation catbed. This section presents the results of these assessment tests. The baseline data will be used later to interpret the results of the hybrid motor hot fire tests.

\section{Evaluation silver catalyst material activation treatments}

A series of initial tests was performed on the silver screen meshes in order to evaluate the effectiveness of the previously described samarium nitrate and nitric acid treatments. The silver screens received either no treatment, nitric acid treatment, or samarium nitrate treatments as described Table 1. Following the solution soak, the screens were heated to approximately $250^{\circ} \mathrm{C}$ for 45 minutes in a small electric resistance oven. For the qualitative pre-treatment evaluation tests, the previously-described catalyst Runckel \#2 pack were assembled with the test silver screens to be evaluated and a thermocouple was attached to the exit of the catalyst pack. The entire assembly was placed in a glass beaker. Figure 18 shows this test configuration. Using an eyedropper $10 \mathrm{ml}$ of $50 \%$ peroxide was flowed through the through the system. Two activated samples with each treatment, nitric acid and samarium nitrate, as well as two untreated control samples were tested.

Figure 19 shows the test results. For each treatment the 2 samples exhibited similar temperature responses; however, the responses due to the various treatments varied widely. The untreated control samples resulted in a rapid temperature rise of approximately $2^{\circ} \mathrm{C}$ as soon as the solution was applied. This temperature rise leveled off and eventually dropped after approximately two and half minutes. This leveling off and drop indicates that the silver screen was less and less active over time. The nitric acid treated samples produced a more gradual change with temperatures varying between approximately $2^{\circ} \mathrm{C}$ and $2.5^{\circ} \mathrm{C}$. Unlike, the untreated sample, this change was more of a smoldering nature with a gradual increase over a nearly 4-minute 
time span. A continuous temperature rise was exhibited throughout the test duration. The samarium nitrate treated samples exhibited little discernable temperature rise. In fact, the first sample tested exhibited a slight temperature loss, likely due to evaporative cooling. The samarium nitrate test results supports the earlier results published by Rusek. ${ }^{48}$ Samarium nitrate is not an effective activation treatment for catalysis when applied to all but very high concentrations of peroxide, $\geq 95 \%$.

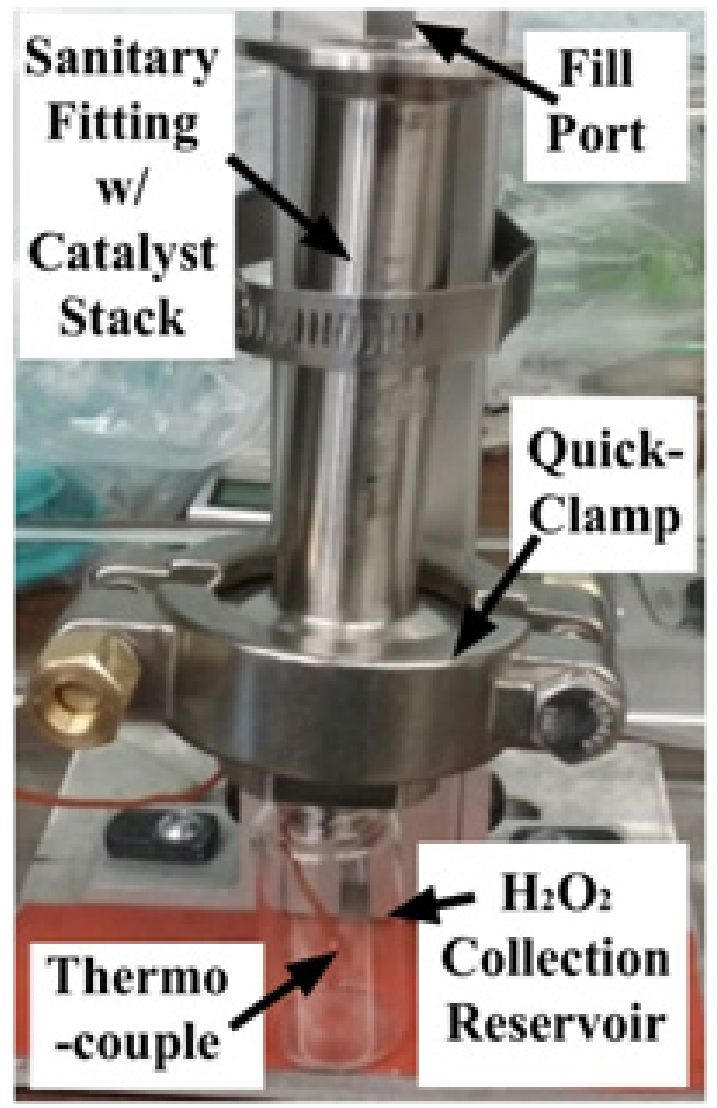

Figure I 8 Test fixture for sensing temperature rise in catalyst stack.

\section{Comparison of nitric acid activated silver screen with $\mathrm{MnO}_{2}$ Treated $\alpha$-alumina Pellets}

Based on the results of the previous section, the nitric acid treated silver screen was down-selected as the control catalyst for the hot fire tests. This catalytic material was to be compared to $\mathrm{MnO}_{2}$ as the low-cost alternative catalyst. Before integrating onto the hybrid motor for hot-fire testing, a series of quantitative tests were performed to compare the activity of the acid-treated silver screen against the $\mathrm{MnO}_{2}$ impregnated $\alpha$-alumina pellets for three low concentrations of peroxide, $20 \%, 30 \%$, and $50 \%$. Table 2 compares the materials, geometries, and peroxide mass concentrations evaluated by these test. Eqs. (8) and (9) were used to calculate the approximate CSI values. Although an effort was made to match the Contact Surface Indices (CSI) of the screens and pellets as closely as possible, in the end the silver screen catalyst presented approximately $5.7 \%$ higher CSI values $\left(648.5 \mathrm{~mm}^{2}\right)$ than did the impregnated pellets $\left(613.5 \mathrm{~mm}^{2}\right)$.
Table I Matrix of activation variables

\begin{tabular}{|c|c|c|c|}
\hline $\begin{array}{l}\text { Activation } \\
\text { treatment option }\end{array}$ & None & $\begin{array}{l}30 \% \text { Nitric } \\
\text { acid bath }\end{array}$ & $\begin{array}{l}\text { I0\% Samarium } \\
\text { nitrate bath }\end{array}$ \\
\hline Time in Bath (s) & -- & 60 & 60 \\
\hline $\begin{array}{l}\text { Rinsed with Distilled } \\
\text { Water }\end{array}$ & No & Yes & Yes \\
\hline Time in Oven (min) & -- & 45 & 45 \\
\hline Number of tests & 2 & 2 & 2 \\
\hline
\end{tabular}

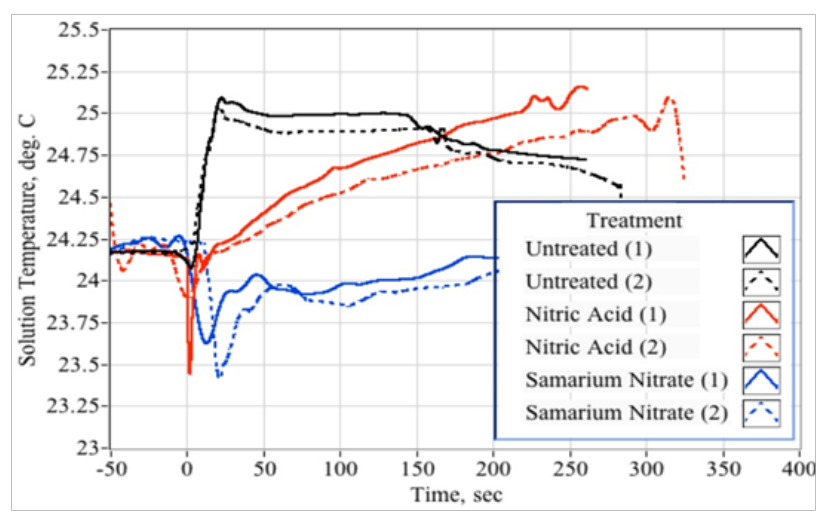

Figure 19 Comparison of temperature rises associated with silver catalys screen pre-treatments.

Figure 20 shows this test apparatus. Here the reaction chamber consisted of a stainless steel quick-clamp tube mounted on a support stand. Sealed feedthroughs allowed the $\mathrm{H}_{2} \mathrm{O}_{2}$ solution temperature measurement using a thermocouple probe. An absolute pressure transducer was fit onto the top of the stainless-steel reaction tank to sense the pressure rise due to the peroxide solution decomposition. A small mesh basket was constructed from non-reactive Monel screens. The basket holding the catalytic materials was secured to the top of the tank via a manual lever. When the lever was opened, the basket falls into the hydrogen peroxide bath and immediately initiates decomposition. The pressure and temperature data were logged and used to calculate the mass concentration and molarity of the solution remaining reaction tank as the peroxide decomposed over time. The sensed rates of reaction and temperature were used to estimate the energy of activation for the catalyzed decomposition.

Analysis methods for reactivity tests: This analysis assumes that the peroxide solution remains below the boiling point of water and that pressure rise is entirely due to the oxygen liberated by the decomposition reaction. The mass of oxygen released is calculated by,

$$
\delta m_{O_{2}}=\left(\frac{\delta p_{O_{2}}}{R_{g} \cdot T}\right) \cdot V_{c}
$$

In Eq. (29) $\delta P_{O 2}$ is the pressure change due to oxygen released from peroxide decomposition, $R_{g}$ is the gas constant for oxygen, $T$ is the temperature of the peroxide solution, and ullage volume of the reaction chamber. For every mole of oxygen generated, two moles of peroxide are decomposed, and two moles of water are released into solution. Assuming an initial solution mass $m$, and an initial peroxide mass concentration $f_{0}$, the solution molality as a function of time is 


$$
\mathbb{M}(t)=\frac{n_{H_{2} O_{2}}(t)}{M_{H_{2} O}(\mathrm{t})}=n_{H_{2} O_{2}}(0)-2 \frac{\left(\frac{\delta p_{O_{2}}}{R_{g} \cdot T}\right) \cdot V_{c}}{M_{w_{O_{2}}}} /\left[\left(\frac{100-f_{0}}{100}\right) m_{0}+\left(\frac{\delta p_{O_{2}}}{R_{g} \cdot T}\right) \cdot V_{c}+\frac{M_{W H_{2} O}}{M_{w_{O_{2}}}}\right]
$$

Table 2 Catalytic activity test matrix

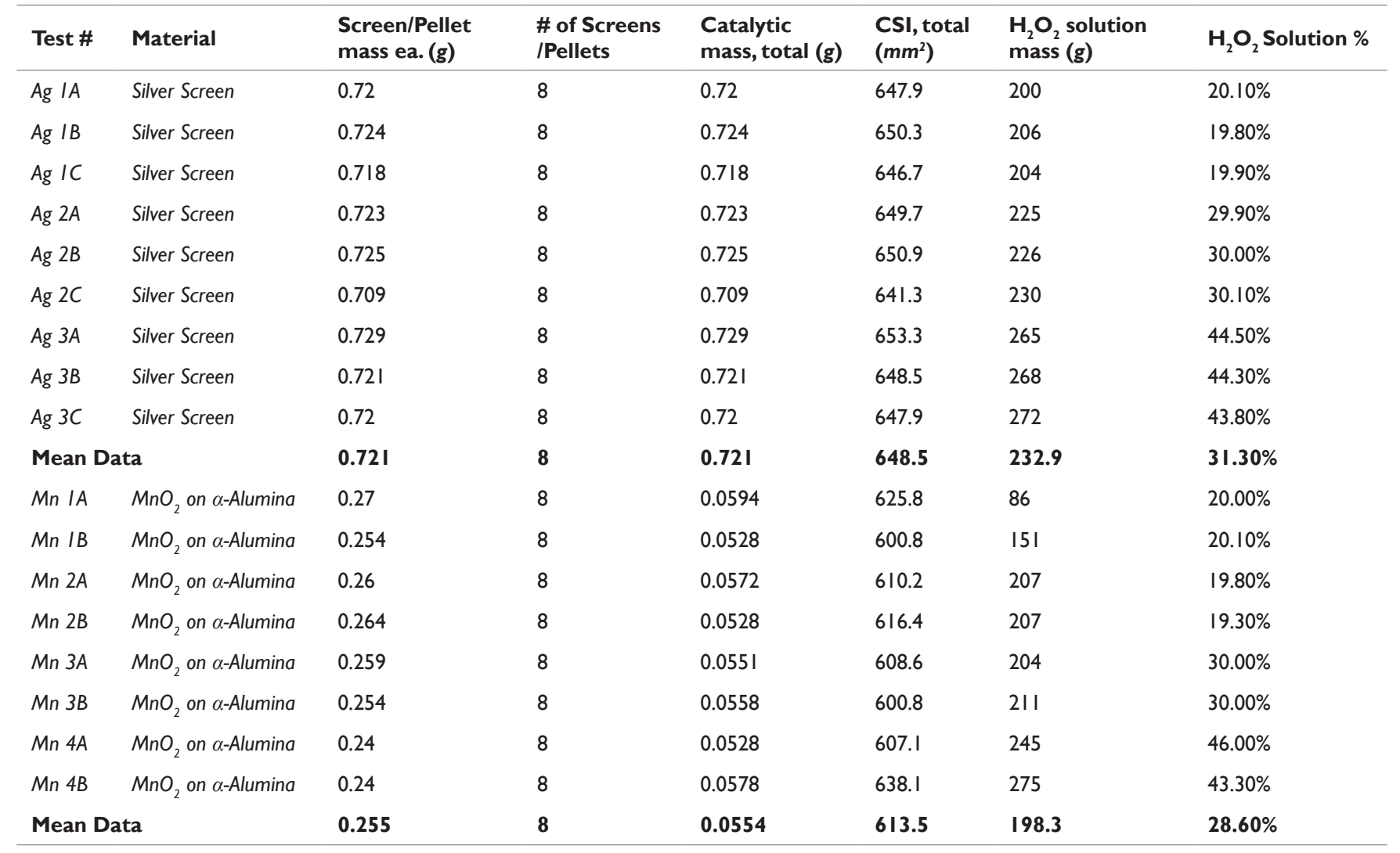

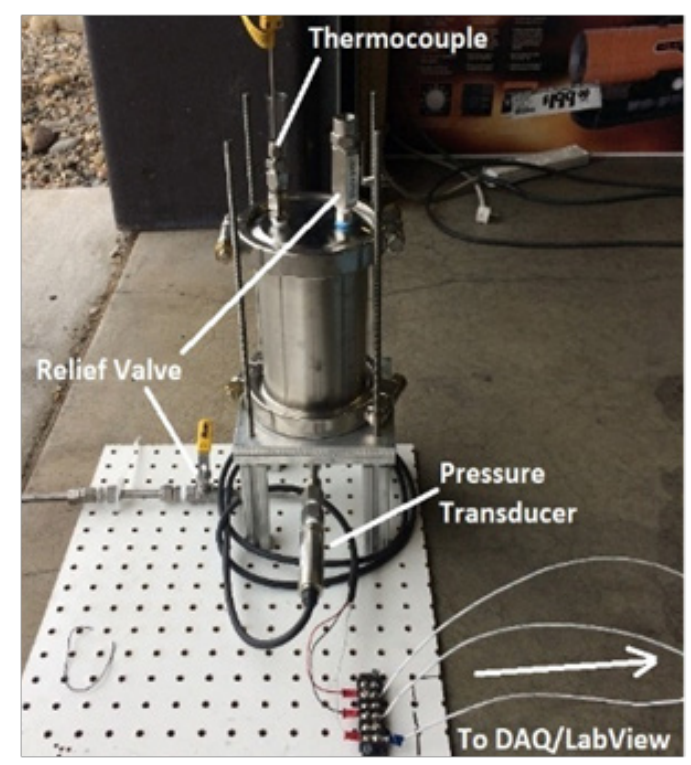

Figure $\mathbf{2 0}$ Test apparatus for measuring pellet catalyst reactivity.
The corresponding mass concentration is

$$
\eta(\mathrm{t})=\frac{m_{\mathrm{H}_{2} \mathrm{O}_{2}}(t)}{m_{\mathrm{H}_{2} \mathrm{O}}(\mathrm{t})+m_{\mathrm{H}_{2} \mathrm{O}_{2}}(t)}=\frac{\mathbb{M}(t) \cdot M_{W_{2} \mathrm{O}_{2}}}{1000+\mathbb{M}(t) \cdot M_{W H_{2} \mathrm{O}_{2}}}
$$

and finally the solution molarity is calculated from the molality by

$$
\Re=\frac{\rho s o \ln }{\left(\frac{1}{M}+\frac{M_{W H_{2} O_{2}}}{1000}\right)}
$$

In Eq. (31) $\rho_{\text {soln }}$ is the current instantaneous density of the peroxide solution, and is function of the solution concentration. As described earlier the peroxide decomposition process here is modeled as a simple Arrhenius-type of first order reaction. ${ }^{49}$ Plotting the rates of reaction against the reaction temperature and curve fitting with a line of the form

$$
\ln \left(\mathrm{k}_{\mathrm{r}}\right)=\mathrm{Y}_{0}-\mathrm{S} \cdot \frac{1}{T}
$$

then, the scale factor and activation energy are calculated by 


$$
\begin{aligned}
& A=e^{\mathrm{Y}_{0}} \\
& \Delta E_{a}=-S \cdot R_{u}
\end{aligned}
$$

Summary of activity test results: Table 3 shows the results where the mean reaction temperature, mean solution molarity, mean molarity slope and reaction rates, from Eq. (17), are tabulated for each test. Figure 21 presents an example decomposition time history taken from test $\mathrm{Mn} 3 \mathrm{~A}$. Plotted are the reaction chamber pressure, the peroxide solution temperature, the released mass of $\mathrm{O}_{2}$ (from decomposition), and the mass concentration of the remaining solution. Because the solution temperature is well below the boiling point of water, it is assumed that the water liberated by the reaction is entirely in liquid form, and only the released oxygen gas contributes to the sensed pressure. Over a period of 150 seconds after the $\mathrm{MnO}_{2}$ is introduced to the $30 \%$ solution, the pressure rises by approximately $56 \mathrm{kPa}$, the solution temperature rises by approximately $3.5 \mathrm{oC}$, and the solution concentration drops to less than $29.4 \%$ concentration. Figure 21 also plots the molarity (e) decay and Molarity (f) rate of decay as a function of time.

For this table, as shown by Figure 21 the molarity time history has been fit with a linear curve in order to estimate the time rate of decay. The process is clearly non-linear, higher order than a simple Arrhenius process; however, Eq. (18) can still be used to compare the relative energies of activation for the two catalytic processes, peroxide decomposition catalyzed by silver screen and $\mathrm{MnO}_{2}$. The Arrhenius plot where the natural $\log$ of the reaction rate is plotted against $1 / \mathrm{T}$, with T expressed in Kelvin units. Note that even though the manganese dioxide coated pellets had a slightly lower CSI compared to the silver screens, the $\mathrm{MnO}$, generally shows a higher decomposition rate as a function of temperature. Table 4 compares the resulting Arrhenius reactivity parameters calculated from these plots using Eqs. (19) and (20). Based on this data the $\mathrm{MnO}_{2}$ pellets appear to significantly increase, by more than $20 \%$, the decomposition activation energy when compared to the nitric acid treated silver screens.

As check on the veracity of the data plotted by Figure 22, the data are overlaid upon analytical results derived by Bramanti et al. ${ }^{36}$ Here the data plotted on Figure 7 and Figure 8 of Bramanti et al., ${ }^{36}$ have been reorganized to plot the logarithm of the reaction rate against the inverse of the reaction temperature. Figure 23 overlays the results from Figure $22 \mathrm{~b}$ on this re-organized data for (a) silver and (b) manganese dioxide. Generally, the comparisons are reasonable with the data of Figure 22 lining up well with the calculations based on CST. For $\mathrm{MnO}_{2}$, the reaction rates present nearly parallel curves indicating the assumed Arrhenius form for the reaction rate-temperature relation is an appropriate assumption. However, the theoretical predictions of Bramanti et al., ${ }^{36}$ show slope-changes as a function of CSI for silver; and this behavior is indicative of a higher-order reaction. The main points to take away from Figure 23 are 1) the reaction rates of Figure 21 are at-least reasonably supported by analytical predictions, and 2) $\mathrm{MnO}_{2}$ appears to offer a moderately larger reduction in energy of activation, when compared to the nitric-acid treated silver screens.

Table 5 lists the scale factors and activation energies calculated for each of the curves plotted on Figure 23a and Figure 23b. The first 6 data columns list the scale factor factors and activation energies calculated from the data of Bramanti et al. ${ }^{36}$ The final column of Table 5 presents data derived from the USU activity test series. As described in the previous paragraph, manganese dioxide catalyzed reaction rates tend to show only a weak variation with the CSI; whereas, the silver-catalyzed reactions show a strong correlation, suggesting a high degree of reaction non-linearity.

\begin{tabular}{|c|c|c|c|c|}
\hline Test No. & T(avg) K & $M(\operatorname{avg})(\mathrm{mol} / \mathrm{l})$ & M(slope) (mol/s) & k (rate) ( $1 / \mathrm{s})$ \\
\hline \multicolumn{5}{|c|}{ Silver Screen Catalyst } \\
\hline $\mathrm{Ag} I \mathrm{~A}$ & 305.2 & 6.2951 & -0.000785 & 0.000125 \\
\hline $\mathrm{Ag} \mid \mathrm{B}$ & 313.2 & 6.2702 & -0.002172 & 0.000346 \\
\hline $\mathrm{Ag} I \mathrm{C}$ & 318.3 & 6.2540 & -0.002519 & 0.000403 \\
\hline $\mathrm{Ag} 2 \mathrm{~A}$ & 304.1 & 9.7923 & $-0.00 \mid 221$ & 0.000125 \\
\hline $\mathrm{Ag} 2 \mathrm{~B}$ & 317.5 & 9.71934 & -0.002901 & 0.000299 \\
\hline $\mathrm{Ag} 2 \mathrm{C}$ & 327.1 & 9.6608 & -0.005337 & 0.000552 \\
\hline $\mathrm{Ag} 3 \mathrm{~A}$ & 302.6 & 15.5336 & -0.001950 & 0.0001256 \\
\hline $\mathrm{Ag} 3 \mathrm{~B}$ & 331.2 & 15.2366 & -0.009523 & 0.000625 \\
\hline $\mathrm{Ag} 3 \mathrm{C}$ & 341.2 & 15.1300 & -0.012523 & 0.000828 \\
\hline \multicolumn{5}{|c|}{$\mathrm{MnO}_{2}$ impregnated $\alpha$-alumina pellet catalyst } \\
\hline Mn IA & 322.2 & 8.0738 & -0.00480 & 0.000662 \\
\hline Mn IB & 342.8 & 9.3149 & -0.00802 & 0.001122 \\
\hline $\mathrm{Mn} 2 \mathrm{~A}$ & 298.6 & 6.0600 & -0.00098 & 0.000165 \\
\hline Mn 2B & 308.3 & 6.0405 & -0.00127 & 0.000215 \\
\hline $\mathrm{Mn} 3 \mathrm{~A}$ & 296.8 & 9.8133 & -0.00172 & 0.000178 \\
\hline Mn 3B & 305.1 & 9.7597 & -0.00232 & 0.000240 \\
\hline Mn 4A & 299.7 & |5.887| & -0.00530 & 0.000184 \\
\hline Mn 4B & 311.7 & 15.0221 & -0.00784 & 0.000362 \\
\hline
\end{tabular}

Table 3 Tabulated results from catalytic activity tests 
Table 4 Summary of measured Arrhenius reactivity parameters for $\mathrm{Ag}$ and $\mathrm{MnO}_{2}$

\begin{tabular}{llllll}
\hline $\mathbf{Z}$ & $\mathbf{Y}_{0}$ & Slope & $\mathbf{A}_{\text {moles/s }}$ & $\Delta \mathbf{E}_{\mathbf{a ~ k J j / m o l - k}}$ & $\Delta \mathbf{E}_{\mathbf{a ~ k J / g - k}}$ \\
\hline Silver Screen Ag & 9.374 & -5457.79 & 11782.8 & 45.38 & 1.335 \\
$\mathrm{MnO}_{2}$ Impregnated $\alpha$ Alumina & 6.425 & -4501.5 & 616.97 & 37.03 & 1.089
\end{tabular}

Table 5 Summary of Arrhenius reactivity parameters for $\mathrm{Ag}$ and $\mathrm{MnO}_{2}$, based on $\mathrm{CSI}$ value

\begin{tabular}{|c|c|c|c|c|c|c|c|}
\hline \multicolumn{8}{|c|}{ Silver screen Ag catalyst } \\
\hline CSI & $914 \mathrm{~mm}^{2}$ & $657 \mathrm{~mm}^{2}$ & $297 \mathrm{~mm}^{2}$ & $137 \mathrm{~mm}^{2}$ & $\mathrm{I} 14 \mathrm{~mm}^{2}$ & $424 \mathrm{~mm}^{2}$ (mean values) & $648 \mathrm{~mm}^{2}$ (USU-derived) \\
\hline$A_{\text {moles/s }}$ & $42,020.60$ & 990.743 & 18.63279 & 0.2687 & 0.02816 & 6.21823 & ||$, 782.8 \mid$ \\
\hline$\Delta \mathbf{E}_{\mathrm{a} \mathrm{kJ} / \mathrm{mol}-\mathrm{k}}$ & 45.26 & 38.15 & 29.04 & 18.34 & 13.19 & 25.93 & 45.38 \\
\hline \multicolumn{8}{|c|}{$\mathrm{MnO}_{2}$ on a alumina catalyst } \\
\hline CSI & $\mathrm{I}, 454 \mathrm{~mm}^{2}$ & $689 \mathrm{~mm}^{2}$ & $612 \mathrm{~mm}^{2}$ & $383 \mathrm{~mm}^{2}$ & $145 \mathrm{~mm}^{2}$ & $657 \mathrm{~mm}^{2}$ (mean values) & $614 \mathrm{~mm}^{2}$ (USU derived) \\
\hline$A_{\text {moles/s }}$ & $16,528.30$ & 4325.02 & 7207.67 & 8042.84 & 5000.64 & 2117.02 & 619.97 \\
\hline$\Delta \mathbf{E}_{\mathrm{a} \mathrm{kJ} / \mathrm{mol}-\mathrm{k}}$ & 43.64 & 41.52 & 43.35 & 44.53 & 45.04 & 41.25 & 37.03 \\
\hline
\end{tabular}
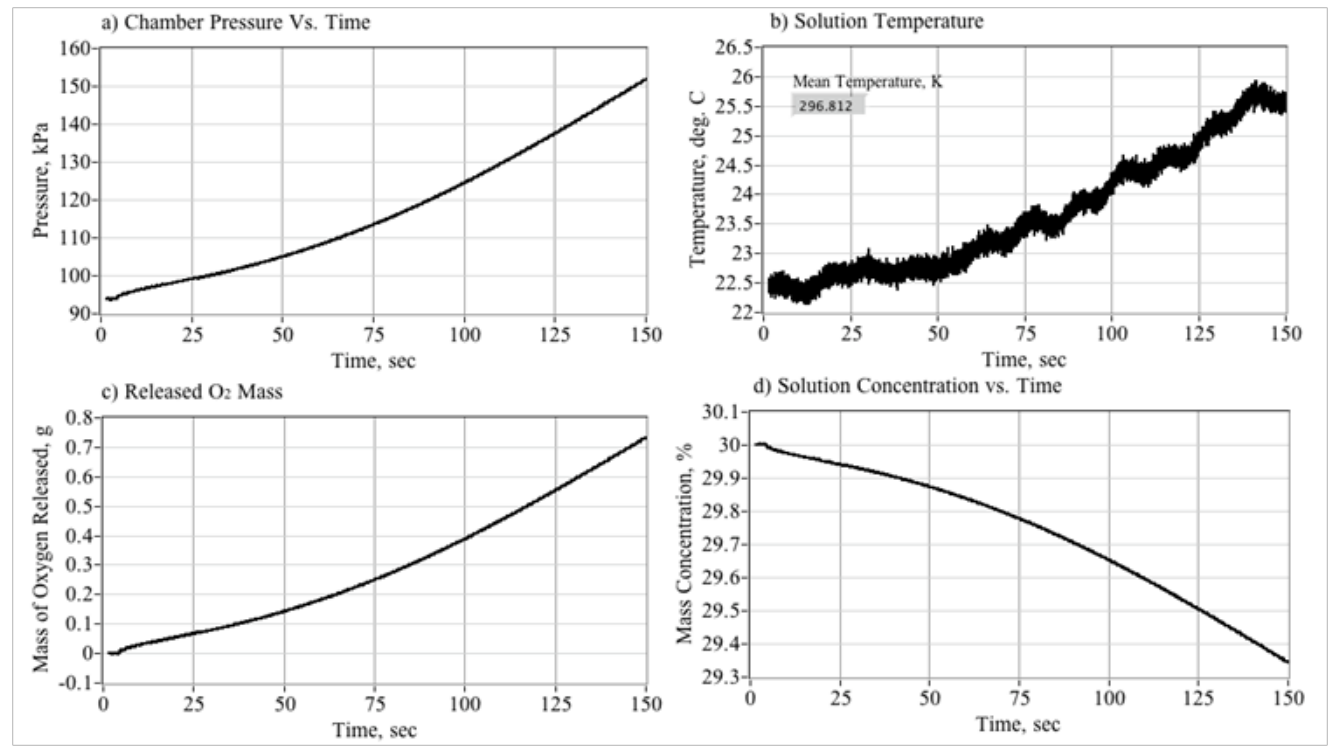

Figure 2 I Decomposition time history after $\mathrm{MnO}_{2}$ catalyst pellets introduced to $30 \%$ peroxide solution.
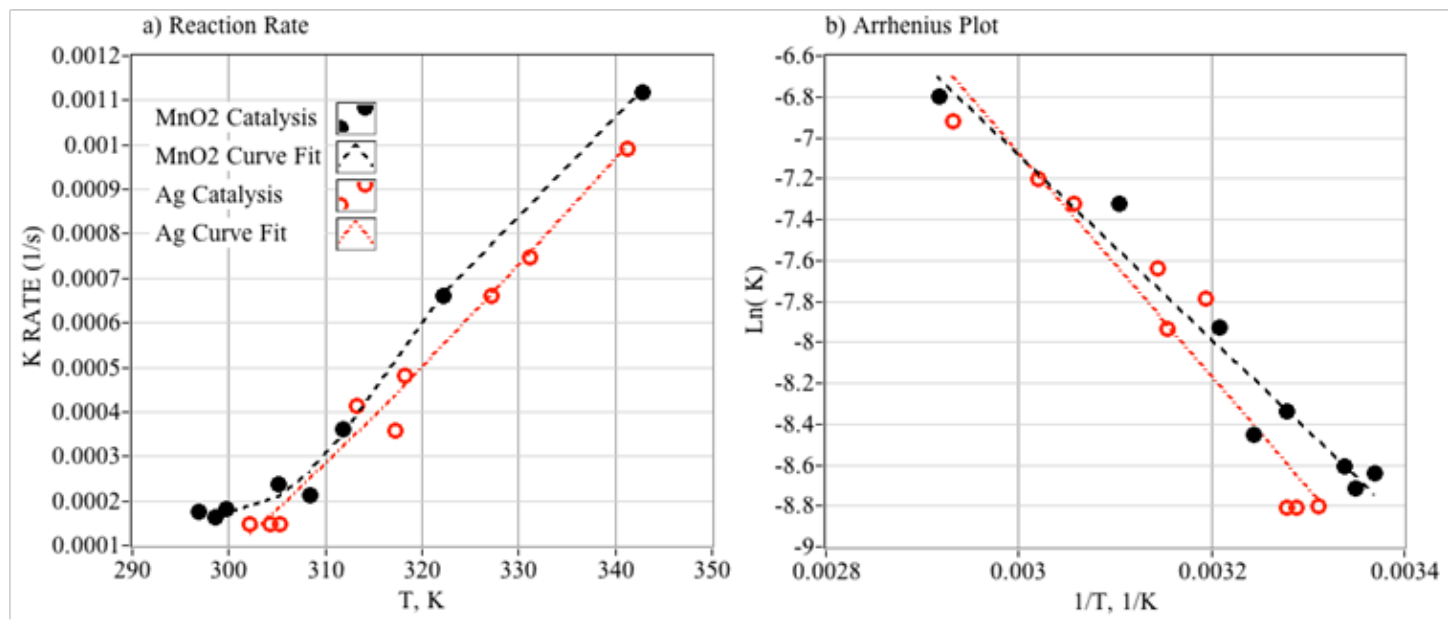

Figure 22 Comparison of $\mathrm{H}_{2} \mathrm{O}_{2}$ reactivity data for $\mathrm{Ag}$ and $\mathrm{MnO}_{2}$ catalysts.

Citation: Whitmore SA, Martinez CJ, Merkley DP. Catalyst development for an arc-ignited hydrogen peroxide/ABS hybrid rocket system. Aeron Aero Open Access J. 2018;2(6):356-388. DOI: I0.15406/aaoaj.2018.02.00069 

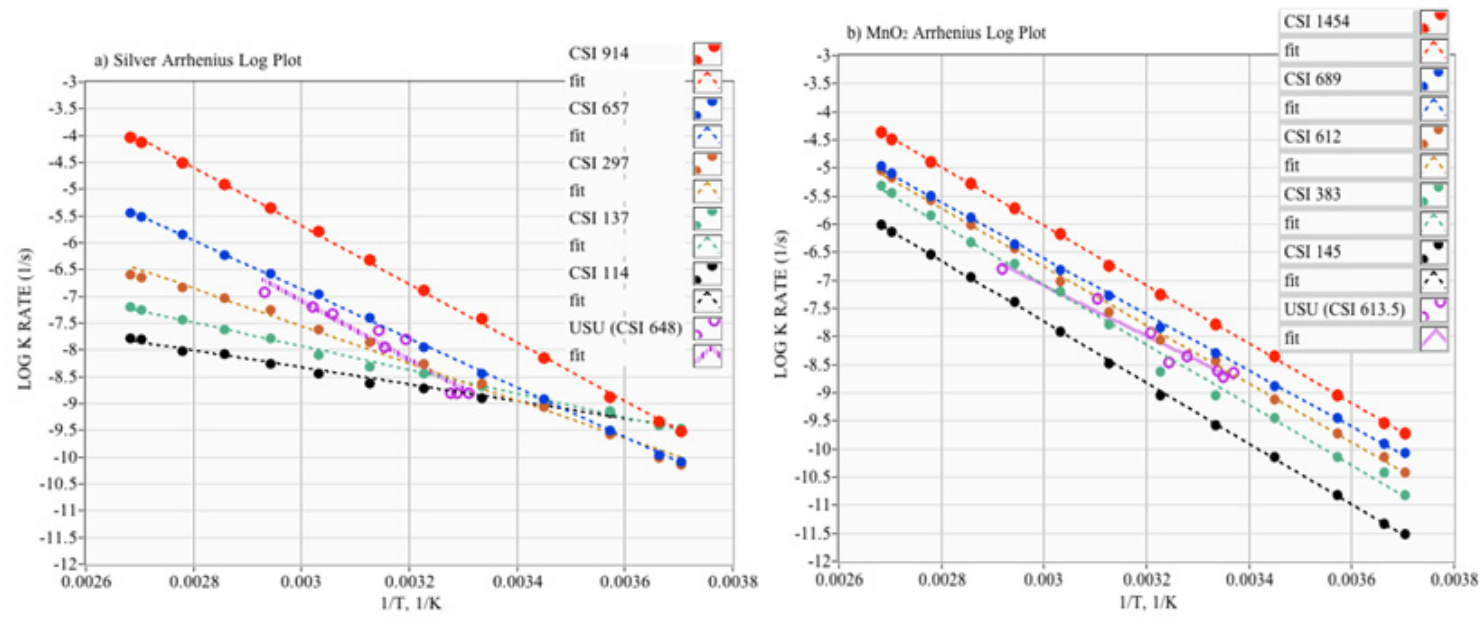

Figure 23 Comparing USU-derived reaction-rate test results of against theoretic calculations. ${ }^{36}$

\section{Warm flow and hot fire test campaigns}

As described previously, a series of hot firings were performed in order to evaluate the performance of the catalyst-augmented ignition system. The primary objective of this test series was to demonstrate that catalytic augmentation can be used to eliminate significantly reduce the "wet-flow" ignition latency depicted by Figure 4 and Figure 5. Prior to testing each of the components nominally designed to touch the oxidizer were "passivated." The thin oxide coating which forms on the metal surface during passivation, renders the surface non-reactive to hydrogen peroxide and prevents the metal from decomposing the peroxide. After the system was passivated, reassembled, and leak check, a preliminary set of "warm-flow" tests were performed to assess the effectiveness of a stand-alone catbed design without the arc ignition system active. Following this test series, the arc-ignition system was activated and the effectiveness of the two previously described catbeds using silver and manganese dioxide were assessed.

\section{Hot fire test instrumentation}

Figure 24 shows the custom test cart layout. The load structure was fabricated using commercially available aluminum "t-slot." The catalyst bed holder of Figure 12 was installed the just upstream of the injector cap. In order to aid catalycity a 100 -watt external band heater ${ }^{16}$ was also installed around the catbed holder. The system uses nitrogen as a pressurant and carbon dioxide to operate all of the pneumatic ball valves. A separate $\mathrm{CO}_{2}$ purge system was also installed. All of the key components are visible and labeled except for the $\mathrm{N}_{2}$ pressurant, $\mathrm{CO}$ purge, and GOX secondary tanks. Figure 24 also shows where the secondary GOX feed line is tee'd into the injector cap. The injector cap lies inside of the thrust chamber and is invisible in this image.

Figure 25 shows the Piping and Instrumentation Diagram (P\&ID) of the System. The system features a 1-liter capacity run tank pressurized by nitrogen gas. The $G O X, N_{2}$, and $\mathrm{CO}_{2}$ tanks are all independently controlled using manually set regulators. The catbed inlet and outlet pressures and the motor head-end chamber pressure are also measured. The flow temperature at the catbed outlet is sensed

${ }^{16} 1$-Piece Mica Insulated Band Heater. with a TC-probe inserted into the flow field. Two calibrated venturi flow meters are installed allowing measurement of both the peroxide and GOX flow rates. The data acquisition and control system was all contained within a single box mounted to the lower shelf of the test cart. Two National Instruments data acquisition and control devices manage motor fire control, and test data logging. Operators and experimenters are remotely located in a secure control room separated from the test area. Communications to the test stand are managed by an operator-controlled laptop via universal serial bus (USB) using amplified extension cables.

\section{Silver-screen catalyst warm flow test results}

The warm flow tests were conducted using exactly the same hardware as depicted by Figure 24 and Figure 25, except without the thrust chamber installed. Also, secondary GOX flow was not applied for the warm flow tests. As described previously, the primary purpose of the warm flow tests was to assess the effectiveness of a stand-alone catbed design. As a safety precaution against an inadvertent fire due highly concentrated peroxide spraying onto the test cart, only solutions of approximately $75 \%$ concentration were used for these tests. A catch bucket was used to capture the any undecomposed peroxide solution exiting the nozzle injector cap. The catbed band heater was used to adjust the entering peroxide temperature from room temperature $20^{\circ} \mathrm{C}$ up to near $90^{\circ} \mathrm{C}$, or just below the boiling point at the test altitude, 1500 meters MSL. Both the silver-screen and $\mathrm{MnO}_{2}$ catalysts were evaluated during warm flow testing. For the silver-screen tests, the Runckel \#2 geometry ${ }^{20}$ was assembled using screens that had been pre-treated in the nitric acid bath.

A series of 8 warm flow burns were performed using the Runckel\#2 catbed design. Table 6 summarizes the warm flow test conditions for the silver-screen catbed. A total of 8 characterization tests were performed. Peroxide flow times varied from as little as 1.2 seconds to over 8 seconds. The 8 warm flow test required that two batches of high grade peroxide be prepared, following the procedures of Section K. The first batch resulted in a concentration of $76.2 \%$, and the second batch resulted in a slightly lower concentration $72.2 \%$. The mass concentrations were calculated by measuring the solution 
volume, weight and temperature, calculating the density, and then comparing to model data presented by Ebelke. ${ }^{47}$ The table lists both $T_{\text {final }}$, the temperature measured at the time the peroxide run valve was closed, and $T_{\text {max }}$, the maximum temperature achieved during the test. The value of $T_{\text {max }}^{\max }$ was used for comparison purposes to allow for heat transfer rates and latencies in the thermocouple measurement systems. The column for \% decomposition lists the ratio of the calculated downstream and upstream mass concentrations, and is a measure of the decomposition efficiency of the catbed. Finally, the columns for L99\% and T99\%, represent the catbed length that would be required for the solution to reach $99 \%$ decomposition, and the temperature that would result from that decomposition. The calculations were all performed using the previously developed catalytic model of Eqn. (33).

Figure 26 shows a typical warm-flow time history profile of a short duration test using the Runckel \#2 silver-screen catbed design. For this run the starting solution concentration is approximately $76.2 \%$. Plotted are (a) catbed inlet and exit pressures, (b) massflow calculated from the venturi flow meter, (c) total peroxide mass through the catbed, and (d) catbed inlet and outlet flow stream temperatures. The total peroxide mass, calculated as the numerical integral of the venturi massflow rate, is curve fit to approximate the mean flow rate for the test, approximately $34.3 \mathrm{~g} / \mathrm{s}$. During this 2 -second run with the inlet pressure set to $150 \mathrm{psia}$, the catbed temperature rises rapidly, within the first $300 \mathrm{~ms}$, from the initial $90^{\circ} \mathrm{C}$ set point to nearly $180^{\circ} \mathrm{C}$. After this initial surge the temperature rise is more gradual reaching slightly above $200^{\circ} \mathrm{C}$ as the run valve closes. The gradual temperature increase and slow pressure drop after the run valve closes is attributable to residual peroxide solution "smoldering" in the catbed. The "buzzsaw" patterns exhibited on both the pressure and massflow traces are likely due to phase change and cavitation within catbed housing. Figure 26e plots the spectrum magnitude of the catbed exit pressure. There exists a prominent peak at approximately $6.5-7 \mathrm{~Hz}$, indicating a strong periodicity to the observed phenomenon. The mean pressure drop across the catbed for this run is only $4.35 \mathrm{psi}$.

Figure 27 presents similar results for a longer duration, 8-second run. Again the temperature rise-rate during the first $300 \mathrm{~ms}$ is rapid and then tapers-off. The peak temperature exceeds $280^{\circ} \mathrm{C}$ at the run valve closing. As with the previously presented data of Figure 26, residual peroxide in the catbed housing continues to smolder after the run valve is closed. Finally, the buzzsaw pressure pattern is still evident; however, the resonant frequency has now dropped to $4.3 \mathrm{~Hz}$. This frequency shift is possibly due to silver oxide deposited on the catbed screens from the nitric acid treatment being swept away by the peroxide flow opening up the flow path area a minor amount. This unsteady harmonic resulting from two-phase flow presents a potential instability hazard for the full hybrid motor system. When the catbed is later coupled with the full hybrid motor system, great care must be taken to ensure that sufficient pressure drop occurs across the injector and into the combustion chamber to ensure that injector feed-coupling does not become an issue. ${ }^{50}$

Because the silver-screen catbed was highly compressed before use, and the compression significantly alters the surface area of the silver screens, it is doubtful that the Arrhenius parameters $(A, \Delta E a\}$ previously measured for loose silver screens dropped into peroxide are likely inaccurate. For the baseline set of model-to-data comparisons to be presented here, the model was "calibrated" to a given warm flow profile by averaging the Arrhenius parameters from Table 5, column 7 , and adjusting the rate constant scale factor $(A)$ such that the sensed exit temperature from the catbed equals the model calculated value for the initial warm flow test. The "calibrated" Arrhenius parameters were then applied to all of the subsequent warm flow burns for the silver-screen catbed.

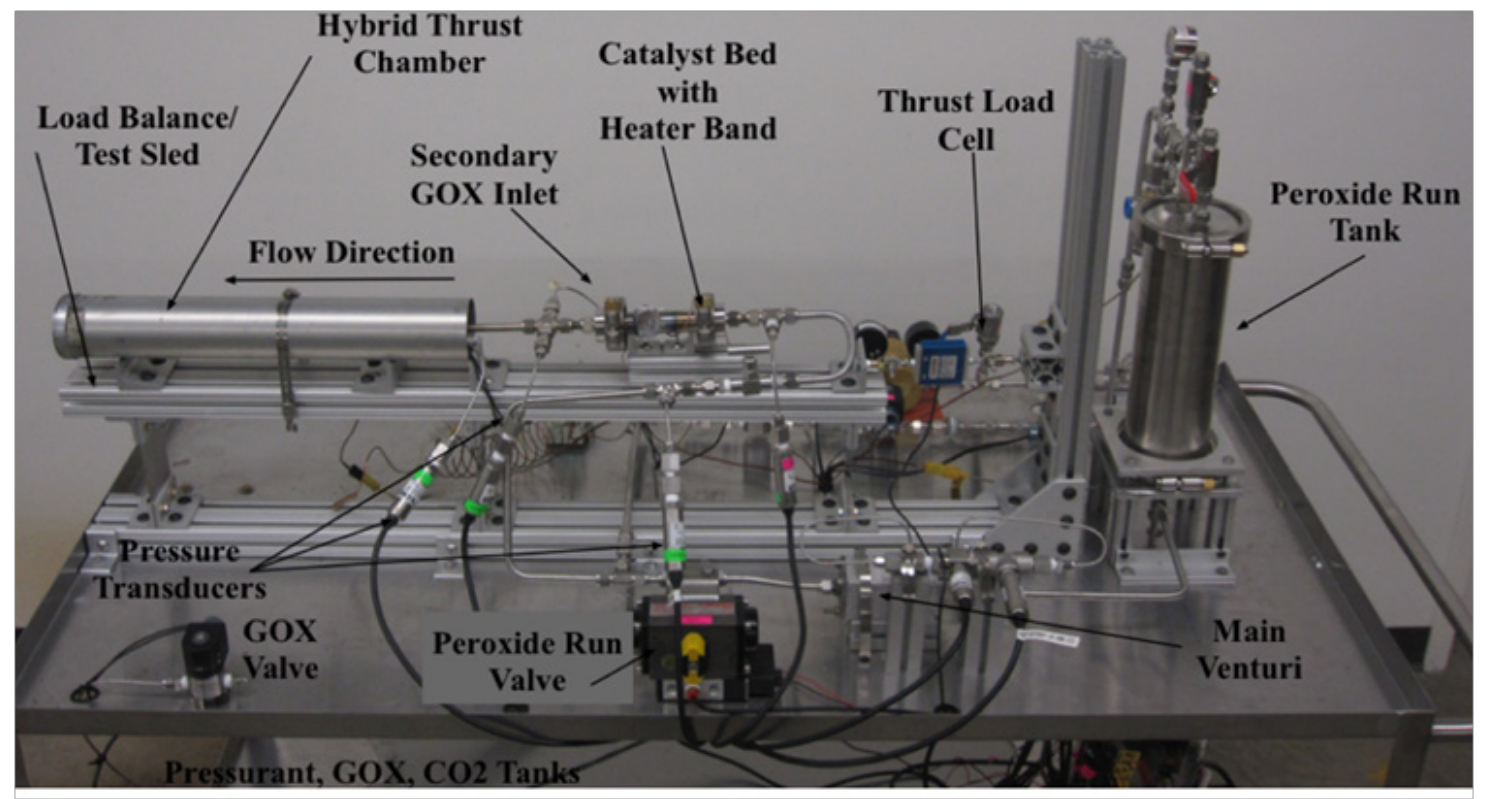

Figure 24 Hot-fire test stand instrumentation layout. 


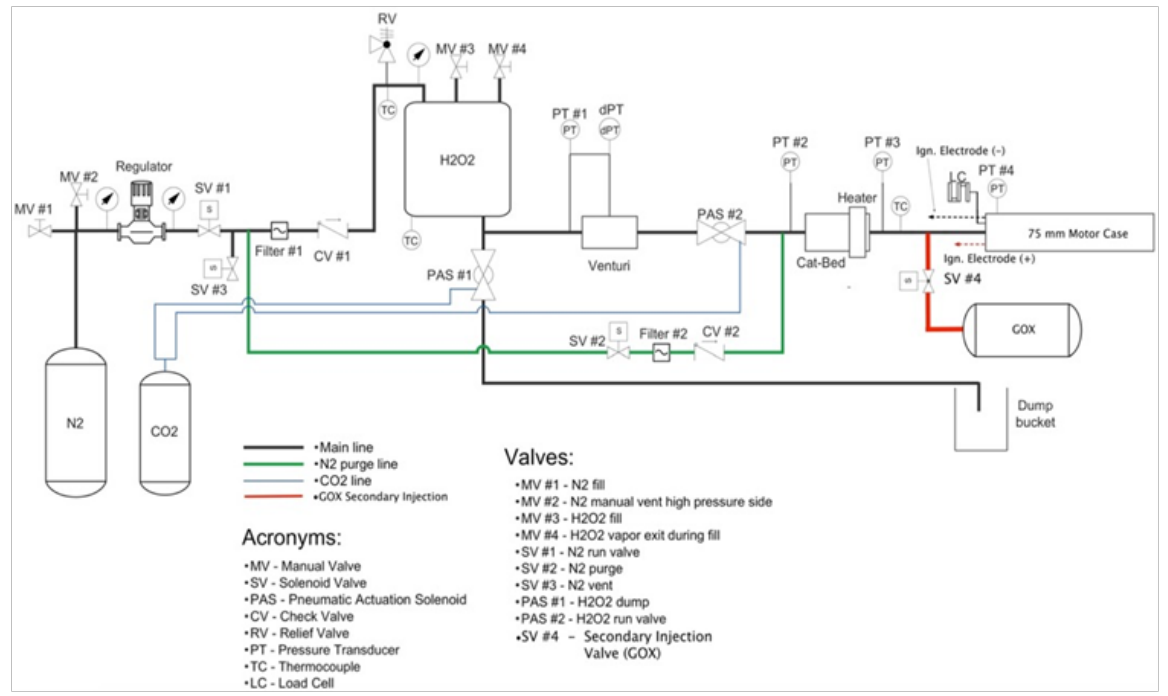

Figure 25 Piping and instrumentation diagram of motor test systems.
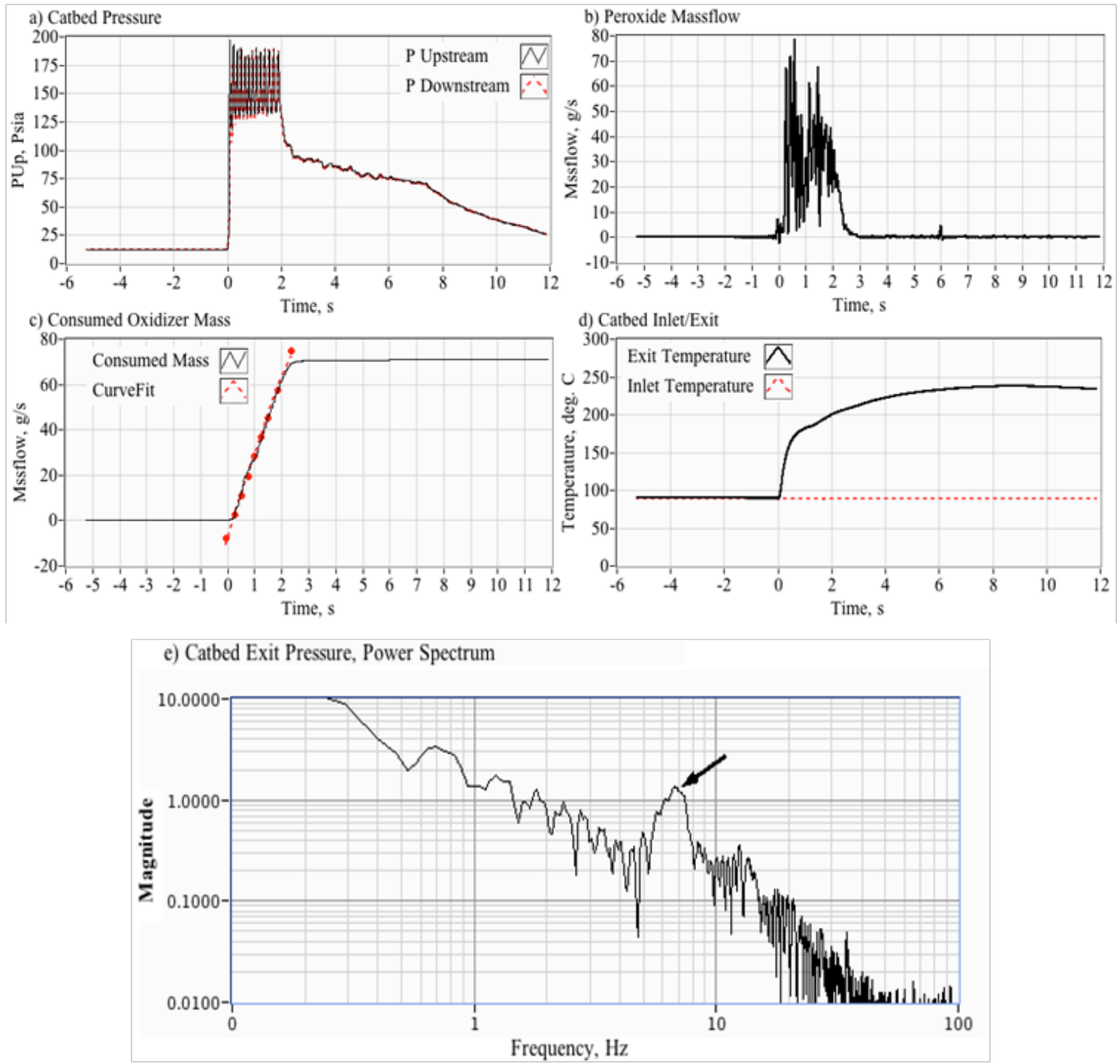

Figure 26 Results from 2-second warm flow test of Runckel \#2 silver screen catbed design. 
Table 6 Summary of silver-screen catbed warm flow tests

\begin{tabular}{|c|c|c|c|c|c|c|c|c|c|c|c|c|c|c|}
\hline $\begin{array}{l}\text { Test } \\
\text { no. }\end{array}$ & $\begin{array}{l}\text { Burn } \\
\text { Time, s }\end{array}$ & $\begin{array}{l}\% \\
\text { Conc. }\end{array}$ & $\begin{array}{l}M_{\text {dot' }} \\
\text { g/sec }\end{array}$ & $\begin{array}{l}T_{0}, \\
\text { deg.c }\end{array}$ & $\begin{array}{l}\mathbf{T}_{\text {final }} \\
\text { deg.c }\end{array}$ & $\begin{array}{l}T_{\max } \\
\text { deg.c }\end{array}$ & $\begin{array}{l}\text { P Cat } \\
\text { Inlet, } \\
\text { psia }\end{array}$ & $\begin{array}{l}\text { P Cat } \\
\text { Outlet, } \\
\text { psia }\end{array}$ & $\begin{array}{l}\text { P Cat } \\
\text { Diff, } \\
\text { psia }\end{array}$ & $\begin{array}{l}\text { Model } \\
\mathbf{T}_{\text {exit' }} \\
\mathbf{C}\end{array}$ & $\begin{array}{l}\text { DP } \\
\text { model, } \\
\text { psia }\end{array}$ & $\begin{array}{l}\% \\
\text { decomp }\end{array}$ & $\begin{array}{l}\text { L99\%, } \\
\text { cm }\end{array}$ & $\begin{array}{l}\text { T99\%, } \\
\text { deg.C }\end{array}$ \\
\hline$|6-1|$ & 1.75 & 76.2 & 54.36 & 21.61 & 33.74 & 65.25 & 136.10 & 131.16 & 4.94 & 58.48 & 5.372 & 7.03 & 56.95 & 317.31 \\
\hline $16-12$ & 2.26 & 76.2 & 35.12 & 73.43 & 183.56 & 212.37 & I 48.35 & 142.69 & 3.66 & 211.90 & 2.685 & 41.1 & 34.26 & 372.81 \\
\hline $16-13$ & 2.4 & 76.2 & 34.35 & 89.23 & 204.60 & 238.10 & 141.32 & 136.97 & 3.05 & 280.75 & 2.093 & 50.6 & 21.39 & 367.22 \\
\hline $16-14$ & 8.3 & 76.2 & 30.92 & 83.57 & 280.28 & 283.29 & |5I.25 & | 48.72 & 2.54 & 283.29 & 1.763 & 41.2 & 23.93 & 367.73 \\
\hline $17-16$ & 8.31 & 72.2 & 45.59 & 48.22 & 28.92 & 97.45 & $|38.3|$ & 134.00 & 4.31 & 89.72 & 3.453 & 9.13 & 41.71 & 315.24 \\
\hline $17-17$ & 1.31 & 72.2 & 36.20 & 93.13 & 208.97 & 248.97 & 184.68 & 182.12 & 2.56 & 243.22 & 2.186 & 34.12 & 26.33 & 364.93 \\
\hline $17-18$ & 1.22 & 72.2 & 18.37 & 77.28 & 184.85 & 224.86 & 214.77 & 213.66 & 1.11 & 310.14 & 0.877 & 61.4 & 24.74 & 359.18 \\
\hline $17-20$ & 8.12 & 72.2 & 29.36 & 67.59 & 216.87 & 227.54 & 193.62 & 191.31 & 2.31 & 189.27 & 1.511 & 28.7 & 29.01 & 344.51 \\
\hline
\end{tabular}
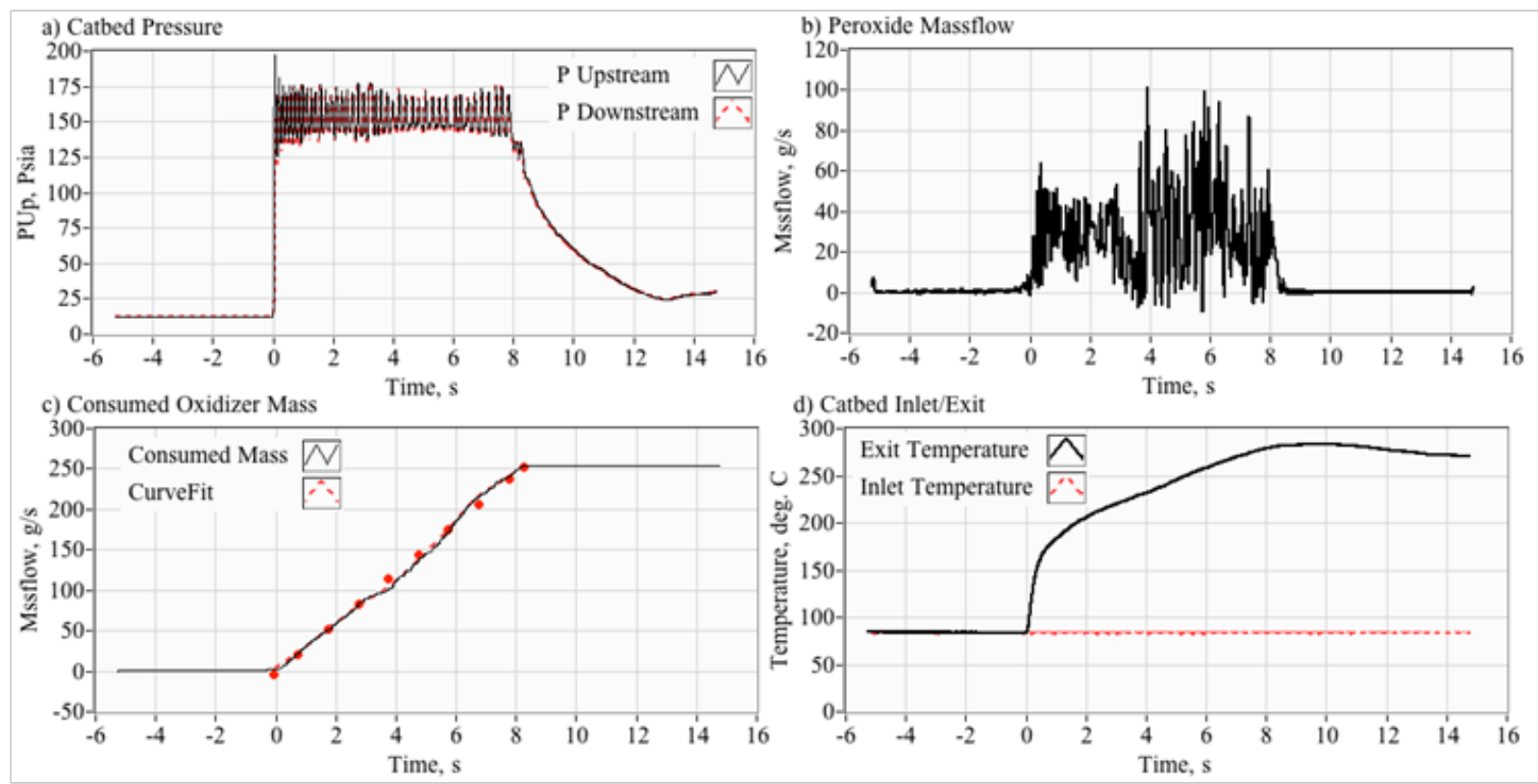

e) Catbed Exit Pressure, Power Spectrum

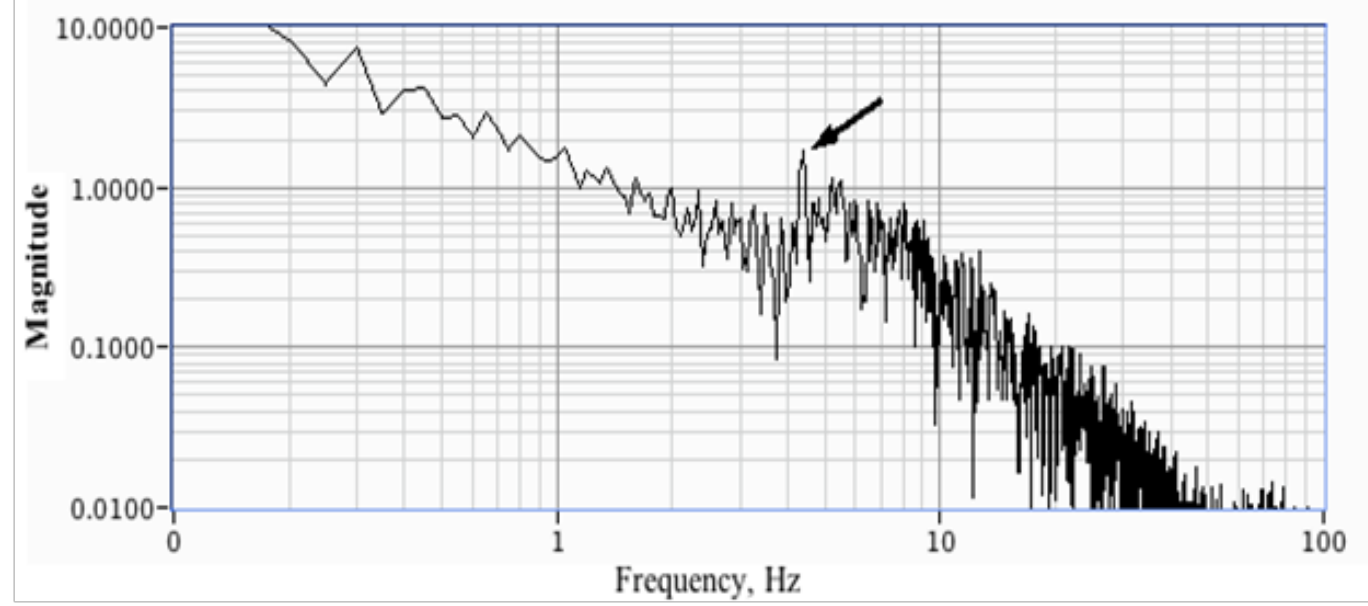

Figure 27 Results from 8-second warm flow test of Runckel \#2 silver screen catbed design. 
Figure 28 presents the results of a typical catbed model calculation for run number 16-14 (From Table 6). Here the Rate of reaction $k_{r}$, mass concentration $f$, Catbed exit temperature $T_{\text {exit }}$, and internal catbed pressure $P_{\text {catbed }}$, are plotted as a function of the longitudinal position along the catbed. Note that for this case the starting mass concentration starts at approximately $76 \%$ and drops to slightly less than $45 \%$ at the catbed exit, $6.15 \mathrm{~cm}$ downstream. This result indicates that the decomposition is only $41 \%$ efficient; thus, the calculated exit temperature $283^{\circ} \mathrm{C}$ is significantly less than would occur with near $100 \%$ decomposition efficiency. Interestingly, the pressure drop is relatively low, slightly less than 2 psia. This result verifies the empirical observations to be presented. Previously, the authors had believed that the low pressure drop across the compressed catbed was due to some sort of anomaly like wall flow bypass around the anti-channel baffles By comparing the result of Table 3 and Figure 22, it appears that the catbed reaction rate constant $k_{r}$ is larger by a factor approximately 10 when compared to the reaction rates that were observed during the reactivity tests for the loose silver screens. This reduced reaction rate appears to be the primary driver behind the observed decomposition inefficiency of the Runkel catbed design.

The data presented by Figures 29-31 further support this assertion. Figure 29a plots the measured and calculated catbed exit temperature and the pressure loss across the catbed against the initial solution temperature. Per the Arrhenius Equation (14), the catbed temperature rise and efficiency and reduced pressure loss as a function of inlet temperature is expected. Also, there exists a high level of agreement between the model predictions and the warm flow observations. This result demonstrates that over the temperature range tested, the decomposition process is rather linear. However, the model also predicts that the Runckel \# silver-screen catbed design is significantly too short to be efficient. Figure 30 plots the calculated catbed decomposition efficiency (fraction of decomposed peroxide) as function of both the inlet solution temperature (a), and also as a function of the water massflow content given by

$$
\dot{m}_{\mathrm{H}_{2} \mathrm{O}}=\left(1-f_{0}\right) \cdot \dot{m}_{\text {solution }}
$$

Note, as shown by Figure 30a that catbed efficiency varies from just below $10 \%$ to a high value of slightly greater than $60 \%$. Clearly the he inlet flow temperature-with sets the initial decomposition ratehas a strong influence on the catbed efficiency. However, it must also be noted that inlet flow water content shows an ever stronger correlation with the catbed efficiency. Figure $30 \mathrm{~b}$ shows this trend. Clearly, the rate of heat removed by the latent heat and heat capacity of the water massflow couples with the temperature influence on reaction time to create this effect. If one extrapolates the data of Figure $30 \mathrm{~b}$, the efficiencies for increasingly stronger solutions concentrations can be projected. Figure 30c shows the results of this calculation. Even above 95\% initial peroxide solution concentration, 90\% decomposition efficiency is not reached. Finally, assuming a $75 \%$ inlet concentration and using the Arrhenius Model the data of Figure 30 predict the catbed length that would be required to achieve $99 \%$ decomposition efficiency and the resulting flow outlet temperatures. Even at low water inlet massflow rates a catbed length of at least $17 \mathrm{~cm}$ is required, regardless of the inlet temperature. The Runckel silver-screen design is simply too short to be efficient except for at very high peroxide mass concentrations. At the lowest mass flows the theoretical temperature of $400^{\circ} \mathrm{C}$ is approached.

\section{Silver screen catalyst hot flow tests}

A baseline series of tests were performed using the \#2 Runckel catbed design, both in stand-alone (no arc-ignition) and augmentation (with arc-ignition) modes. These baseline results demonstrated that the arc-ignition is still necessary in order to achieve full hybrid combustion for moderate peroxide concentrations $(<91 \%)$. Both nitric acid and samarium nitrate treated silver screens were tested; however, but for the one samarium nitrate test performed nearly no catalytic activity was observed, and as a cost saving measure no further tests were performed using this treatment method.

Silver-screen catbed tests with no arc-ignition: As mentioned previously, the first tests in this series attempted to achieve ignition using only the \#2 Runckel silver screen catbed with the arc-ignition system inactive. Example catalyst-only test results are presented by Figure 32 and Figure 33. Figure 32 shows the results of 6 hot-fire tests performed using the silver screen catalyst pre-treated using nitric acid. For these tests the peroxide concentrations varied from $81 \%$ to $91 \%$, with a mean value of $86,4 \%$. For the initial run the catbed preheater was inactive, and was adjusted to higher heating levels for each subsequent run. Plotted are (a) Downstream Catbed Pressure, (b) Chamber Pressure, (c) Catbed Exit Temperature, and (d) the Calculated Decomposition Efficiency. The theoretical 100\% decomposition temperature for an $86.4 \%$ concentration peroxide solution is approximately $618^{\circ} \mathrm{C}$. The decomposition efficiency (d) is estimated by comparing the catbed exhaust temperature against the temperature-efficiency data plotted on Figure 15.

Increasing levels of catalyst activity were observed as the heater output wattage was raised. However, except for one case, the chamber pressure does not build up. Based on these results, it is concluded that liquid water has re-condensed and the nozzle exit remains un-choked. As the plume exits the injector and rapidly expands entering the combustion chamber, the plume super cools and temperature drops below $100^{\circ} \mathrm{C}$. The "soaked" fuel grains will simply not ignite. For all 6 of the trials plotted by Figure 32, liquid peroxide and condensed water were observed shooting from the nozzle exit.

Figure 33 shows the results of a follow on test using a 91\% peroxide solution-the maximum concentration allowed by the project safety rules. The catalyst bed heater was not used for this run. A thermocouple was also placed at the nozzle exit to record the exhaust temperature. In this case, using the higher concentration peroxide pushed the catbed exit temperature up to over $500^{\circ} \mathrm{C}$, and the chamber pressure rises to greater than 100 psia. However, the decomposition efficiency does not overcome the cooling due to plume expansion into the chamber, and flow temperatures were still insufficient to pyrolyze the hybrid fuel. Figure 33c shows this comparison. The combustor temperature rises to slightly less than $300 \mathrm{oC}$, but it still well below the nominal ABS pyrolysis temperature of $300 \mathrm{oC}$. Consequently, only a smoldering "monopropellant" type of burn occurs. Achieved thrust levels for this burn were still low, less than $40 \mathrm{~N}$ compared to the $140 \mathrm{~N}$ achieved for full combustion, as shown by Figure 4 . Based on the data of Figure 16 and assuming a 90\% decomposition efficiency, a concentration of $98-99 \%$ peroxide would be necessary to raise the chamber temperature sufficiently to pyrolysis the fuel. Fuel pyrolysis is necessary for full combustion to be achieved. 

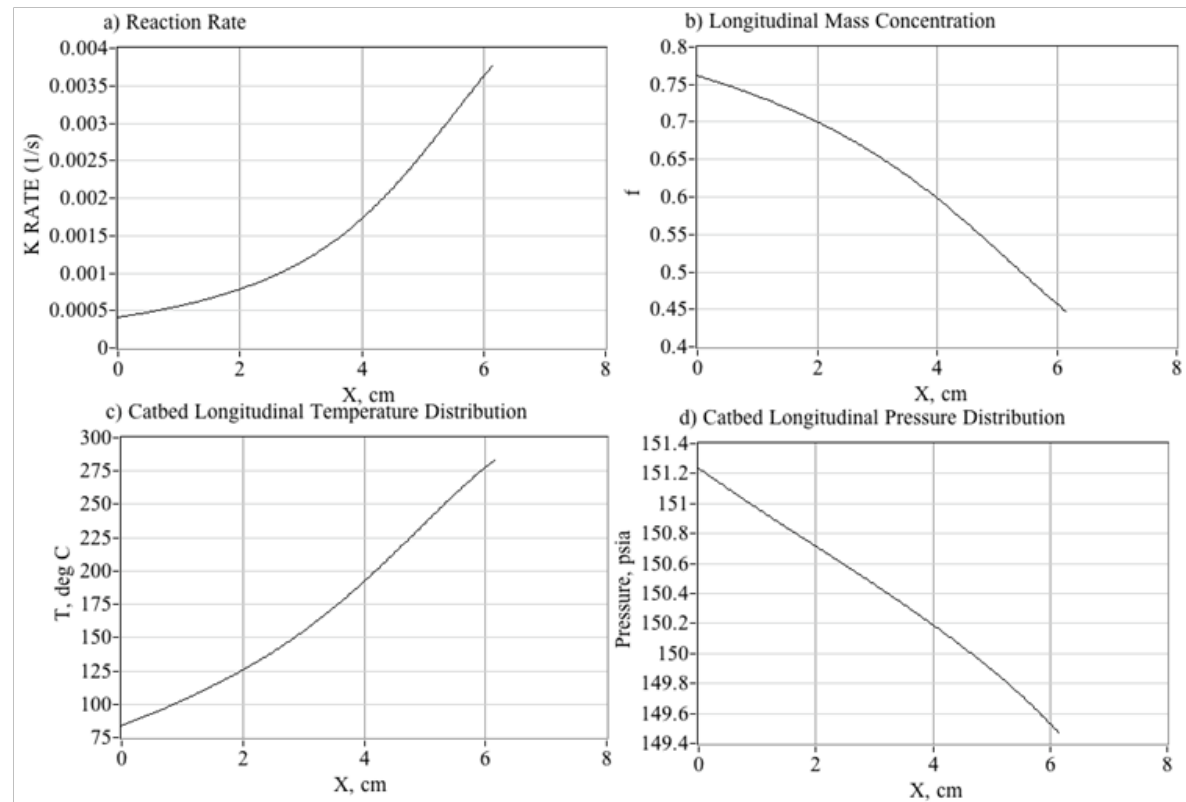

Figure 28 Silver catbed model calculations, showing key Arrhenius parameters and longitudinal temperature and pressure distributions.
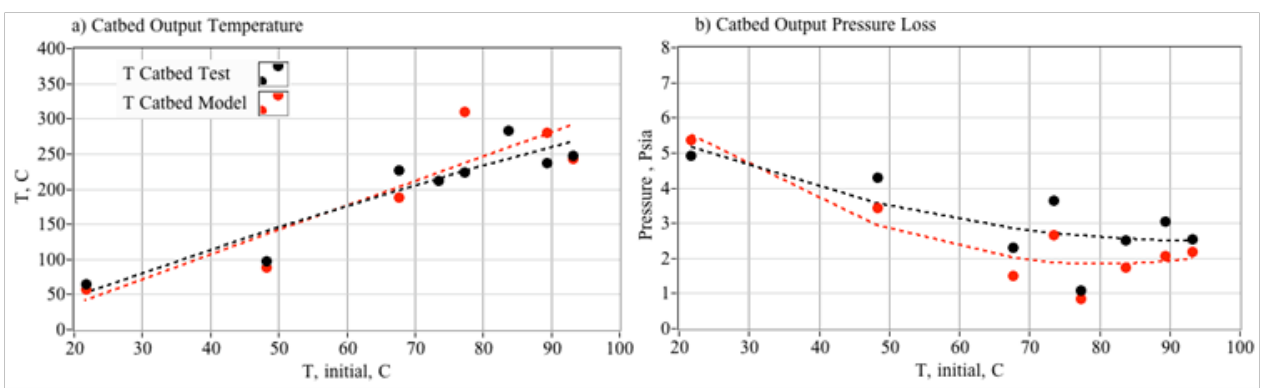

Figure 29 Comparison of measured and modeled catbed outlet temperature and pressure drops.
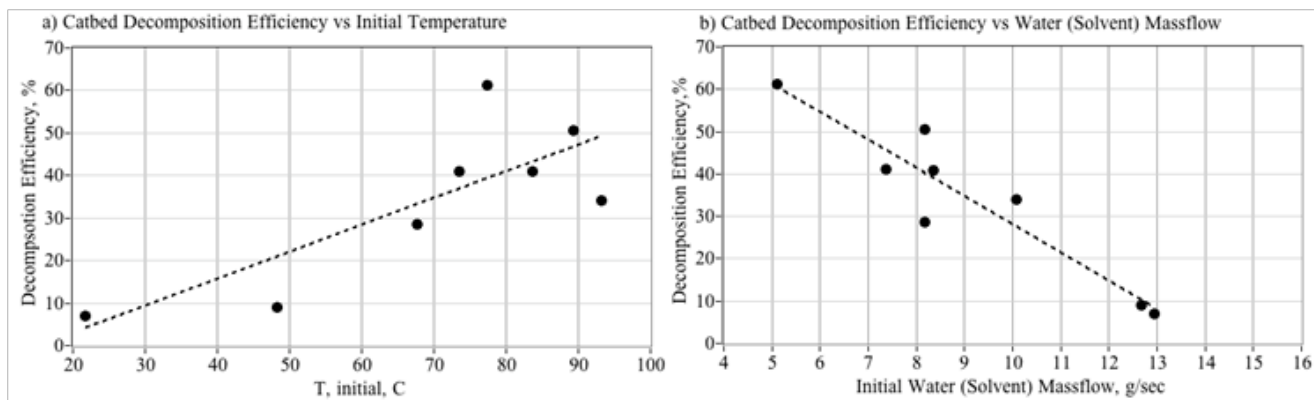

c) Projected Catbed Decomposition Efficiency vs Total Massflow

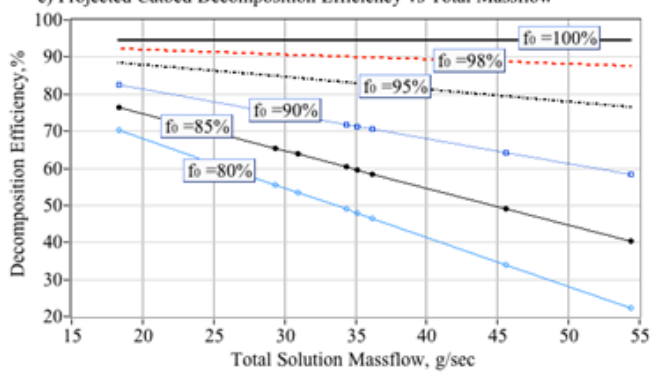

Figure 30 Effects of inlet solution temperature and mass concentration on solution efficiency. 

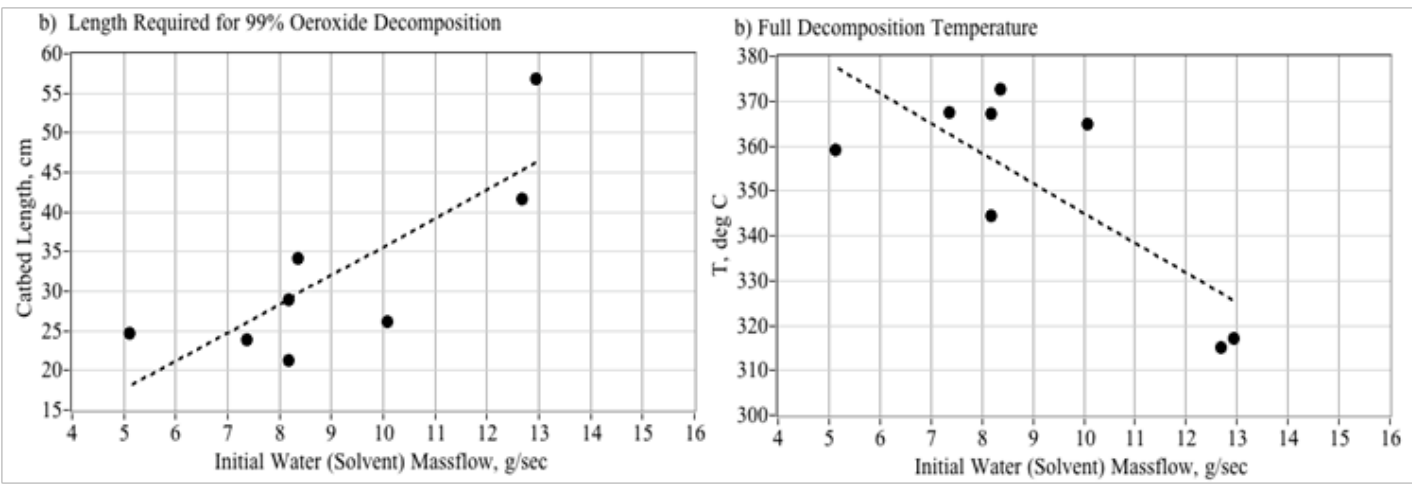

Figure 3 I Required length for silver-screen catbed for $99 \%$ decomposition of peroxide solution and resulting decomposition temperatures.
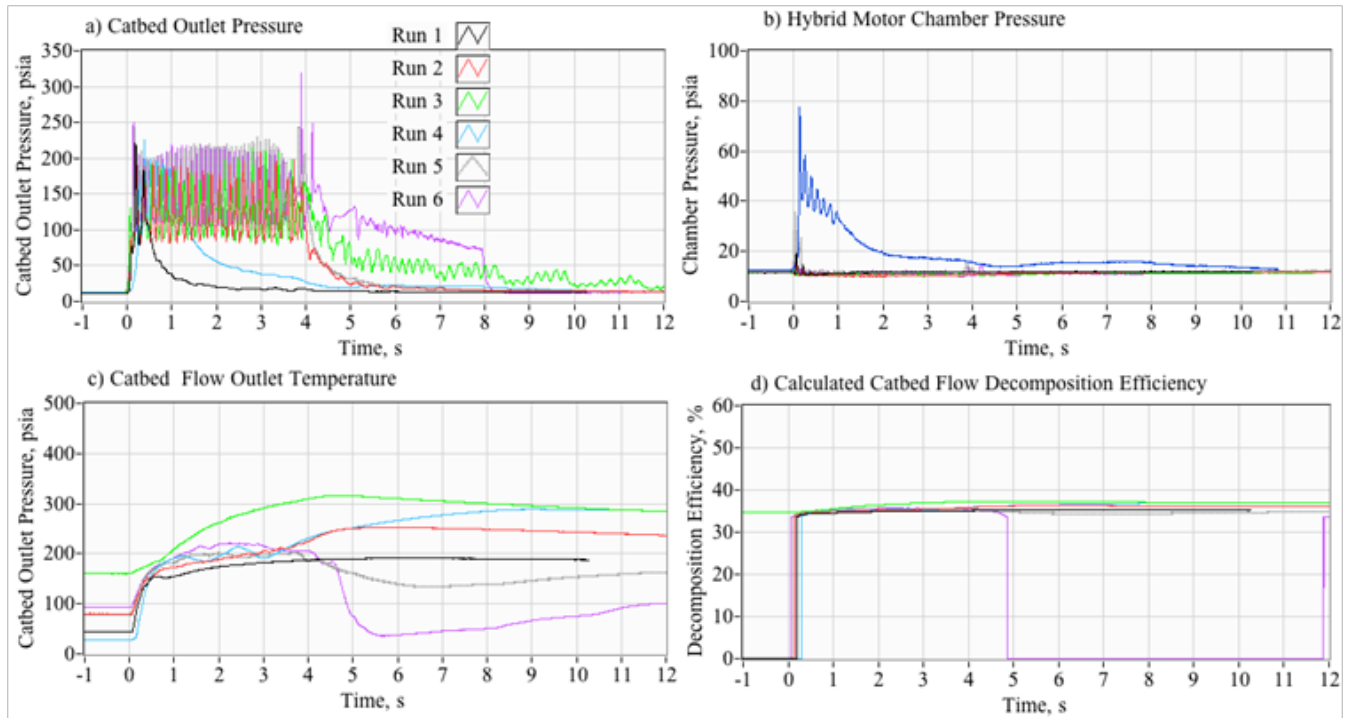

Figure 32 Unsuccessful hot-fire ignition tests of $75 \mathrm{~mm}$ hybrid with $86.4 \%$ peroxide, nitric acid treated silver catalyst bed design (Runckel \#2).
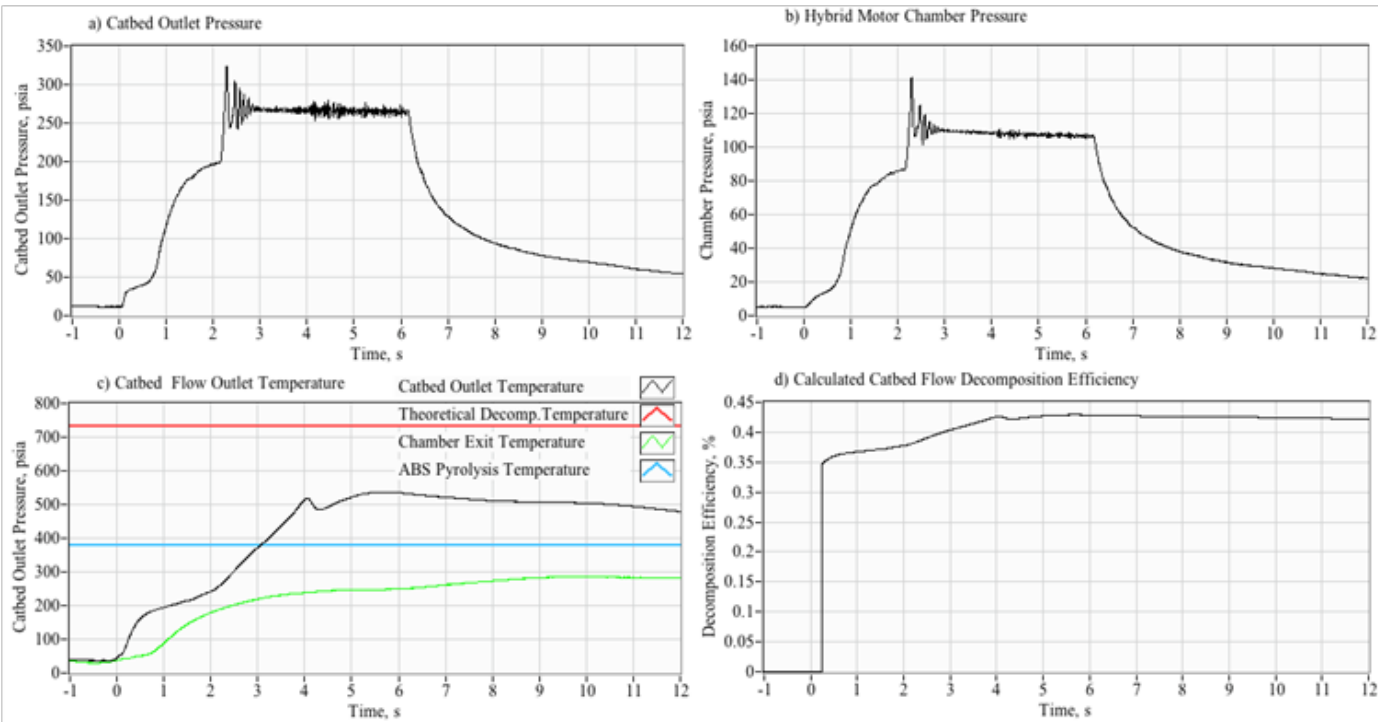

Figure 33 Unsuccessful hot-fire ignition test of $75 \mathrm{~mm}$ hybrid with $91 \%$ peroxide, nitric acid treated silver catalyst bed design (Runckel \#2) 
Thermal-catalytic silver screen hot-fire tests with arc-ignition augmentation: Following the unsuccessful silver-screen catalyst-only tests whose results were presented in the previous section using the silver screen catbed, a series of tests were performed where both the catbed and arc ignition system were installed and operable. Because the GOX pre-lead has the effect of significantly raising the chamber pressure before peroxide injection, the entering decomposition products do not super-cool and return to liquid phase. As a result, these tests achieved considerably more success. Multiple successful full-burns were achieved with various levels of GOX-pre-lead.

Figure 34 shows an early cold-start test result where the catbed heater was not activated. Here the ignition spark precedes the GOX flow by 1-second, and the GOX flow-controlled by a separate solenoid run valve-was set to pre-lead the peroxide flow by approximately 3 seconds. Plotted are time histories of (a) thrust, (b) chamber pressure, (c) total, main, secondary, and fuel massflow, (d) specific impulse, (e) ignition input wattage and total ignition energy, and (f) oxidizer and total massflux. The time scale has been configured to have the zero point coincide with opening of the peroxide run valve. Note on Figure $34 \mathrm{a}$ and Figure $34 \mathrm{~b}$ that once the peroxide valve opens there is an immediate rise in chamber pressure and thrust level. The addition of the catalyst augmentation significantly reduces the ignition latency and the flood of unburned peroxide into the thrust chamber compared to arc-ignition only data presented by Figure 4 . The $I_{s p}$ plot shows that there still is some residual burn inefficiency. The initial burst of unburned $\mathrm{H}_{2} \mathrm{O}_{2}$ results in an approximately 5-8 percent reduction of the cumulative $I_{s p}$ compared to the instantaneous value.

Also note that even though the same upstream regulator setting approximately 400 psia used was identical to that used for Figure 4 , the achieved chamber pressure and thrust levels are reduced by approximately $45 \%$. This drop is a result of stagnation pressure losses across the catbed. The initial GOX flow ignition, followed by the complete peroxide ignition is clearly visible in the thrust (a) and chamber pressure (b) plot contours. Once $\mathrm{H}_{2} \mathrm{O}_{2}$ "ignition" results the thrust and chamber pressure levels jump by factors between 12-15 times. The relative $\mathrm{H}_{2} \mathrm{O}_{2}$ to $\mathrm{GOX}$ massflow varies between ratios of approximately $10-20$.

Figure 35 shows a repeat test of the previous configuration, except now the regulator outlet pressure has been set to max outlet setting of 500 psia and the catbed heater has been set to a full wattage output, approximately a $400^{\circ} \mathrm{C}$ temperature level. The thrust and chamber pressure levels are still a bit lower than were achieved by the burn of Figure 4, again primarily due to pressure losses across the catbed. The ignition transient has now been substantially reduced, and only a short duration spike in the unburned peroxide mass flow is noted in Figure $35 \mathrm{c}$. Also note that the peroxide flow is considerably more stable indicating that the hot catbed has the effect of eliminating the majority of the two phase flow issues. Finally, it is noted that the cumulative Isp is now just $2-3 \%$ lower than the peak instantaneous value, indicating that very little total impulse has been lost due to the ignition transient. Also the overall $I_{s p}$ is approximately $5 \%$ higher.

Currently, with the silver screen catalyst appears that a full catbed reheat to at least 300 degrees is required in order to achieve full ignition augmentation. Unfortunately, this preheat performance enhancement does come at a considerable energy cost. As mentioned earlier entire silver-screen catbed stack in this case weigh approximately 620 grams. Assuming a mean specific heat of $0.4 \mathrm{~J} / \mathrm{g}-\mathrm{K}$ and a heating efficiency of $05 \%$, then heating the stack from ambient temperature to $400^{\circ} \mathrm{C}$ consumes approximately $180 \mathrm{~kJ} .{ }^{17}$ This consumed-energy value is nearly 5 orders of magnitude greater than the required input energy or the arc ignition system.

\section{Warm flow catbed-only catbed tests with manganese dioxide}

The pellet-bed catalyst testing is significantly immature at this point in the project. Before a hot fire was conducted with the $\mathrm{MnO}_{2}$ pelletbed catalyst, two "warm flow" catbed-only tests were performed. The hybrid combustion chamber was removed and the tests consisted of running the peroxide solution through the catalyst bed and injector, and then dumping to ambient conditions. The goal was to establish a baseline for the outlet temperature and pressure drop across the catalyst bed. These tests had been previously performed by Whitmore et al., ${ }^{51}$ for the Runckel silver screen catbed design, and will not be reported here. The warm flow tests were repeated twice with the same catalyst materials in order to identify any potential deactivation issues and assure that the pellet beds survive the temperatures of decomposition.

Figure 36 presents a typical result where the upstream and downstream catbed pressures as well as the pressure difference are plotted by Figure 36a and the decomposition temperature at the catbed exit is plotted by Figure 36b. Figure 35c plots the peroxide massflow. The "burn" lasted approximately 3 seconds, and mean peroxide massflow of approximately 60 grams/s was reached. The heater band was not used for these tests. The thermocouple for this test was not positioned directly in the flow path, and this small temperature rise of slightly above $90^{\circ} \mathrm{C}$ is a likely cause of this bad positioning. From observation, the housing of the catalyst bed got quite hot and was visibly smoking at the test's end. Interestingly, although there is the expected pressure drop across the catbed during the main flow, there is a pressure rise across the catbed as the burn completes. This rise is caused by the residual peroxide strongly decomposing along the length of the catbed lasts for several seconds after the burn. The liberated oxygen gas from decomposition likely results in the pressure rise. Clearly, at least moderate catalytic activity is indicated by the observed temperature and pressure traces.

A second warm flow replicating the conditions of the first burn was performed, with the only difference being that the catalyst material was reused from the first burn. Figure 37 compares the appearance of the pellets following burn two, with a freshly coated set of pellets. The reacted pellets took on a lighter brown color, in contrast to their initial dark brown color. This change in color is indicative of a change in their catalytic effectiveness. The warm flow burn 2 results, plotted by Figure 38 , verify this drop in effectiveness; only minor pressure drop and temperature increase were experienced. Whether this reactivity remains constant after the initial drop or continues dropping is a subject of potential future research. Clearly, lifetime durability is a potential issue with this catalyst design.

\section{Initial manganese dioxide pellet bed hot flow test results}

To date only two hot fire tests of the $\mathrm{MnO}_{2}$ pellet augmentation catalyst have only been performed. Results have only been marginally successful. Figure 39 shows an example test firing behavior. The top image occurs shortly after the peroxide is injected into the combustion

\footnotetext{
${ }^{17}$ This value is consistent with the observation that it took approximately 30 minutes or 1800 seconds for the 100 -watt band heater to heat the installed catbed fixture to a maximum temperature of $400^{\circ} \mathrm{C}$.
} 
chamber. This wet burn mode lasts only a few microseconds. The bottom is the steady state burn as full combustion is achieved. Although, this burn sequence looks normal, inspection of the time history plots reveals the pathological nature of the burn. Figure 40 presents this data. Plotted are (a) directly measured (load cell) thrust and thrust calculated from chamber pressure using the De Laval flow equations, (b) chamber pressure, (c) GOX, peroxide, nozzle exit, and fuel massflow rates, (d) cumulative and instantaneous specific impulse values as calculated using both measured and calculated thrust levels, (e) input power and energy levels, and (f) the oxidizer and total massflux through the fuel port. Although the ignition appears to proceed normally with a sharp initial rise in chamber pressure, shortly after full ignition there is a significant drop off in chamber pressure, thrust, and oxidizer massflow.

Post-test examination of the catbed revealed the cause of this anomalous behavior. Once the catbed housing bed was opened the residue of multiple crushed pellets was discovered. This debris had the effect of plugging the catbed flow path, starving off the peroxide flow to the combustion chamber. In fact, the plug was so substantial that the catbed remained sealed at high pressure. Figure 41 presents data to support this statement. Figure 41a compares the chamber pressure to the internal catbed pressures. Notice that even after the flow has terminated the internal catbed pressured remain high, at nearly 350 psia. Similarly, the internal catbed temperatures continue to rise, even after flow termination. This rise is indicative on peroxide trapped inside of the catbed housing continuing to react with the $\mathrm{MnO}_{2}$. This behavior has occurred in both $\mathrm{MnO}_{2}$ catbed test, and is indicative of a structural design flaw in the $\mathrm{MnO}_{2}$ catbed configuration. On a positive note, the Manganese Dioxide catalyst shows excellent activity properties, even when the support material is crushed.
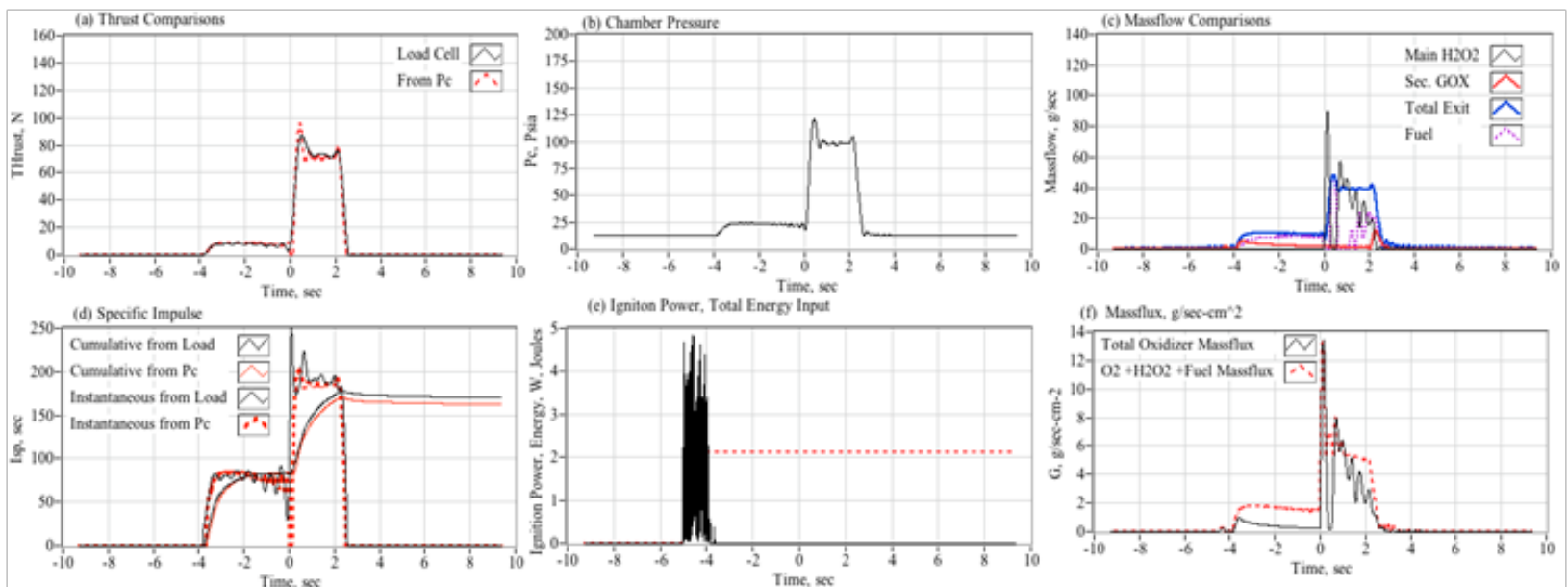
(e) Igniton Power, Total Energy Input
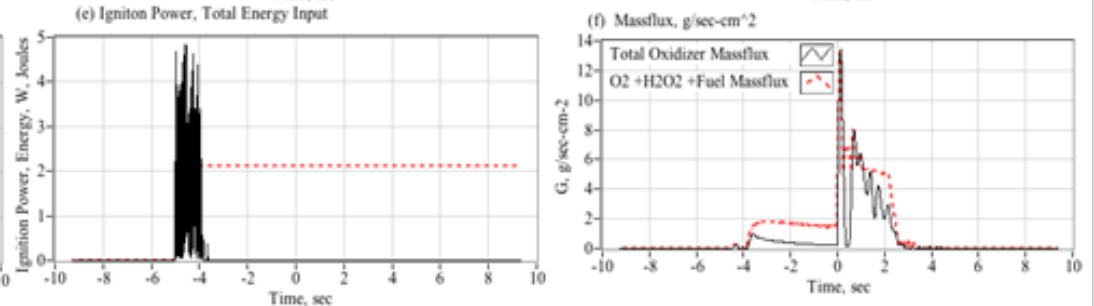

Figure 34 Preliminary hot-fire test using $87 \% \mathrm{H}_{2} \mathrm{O}_{2}$ with silver catbed augmenting GOX arc-ignition, pre-heat inactive.
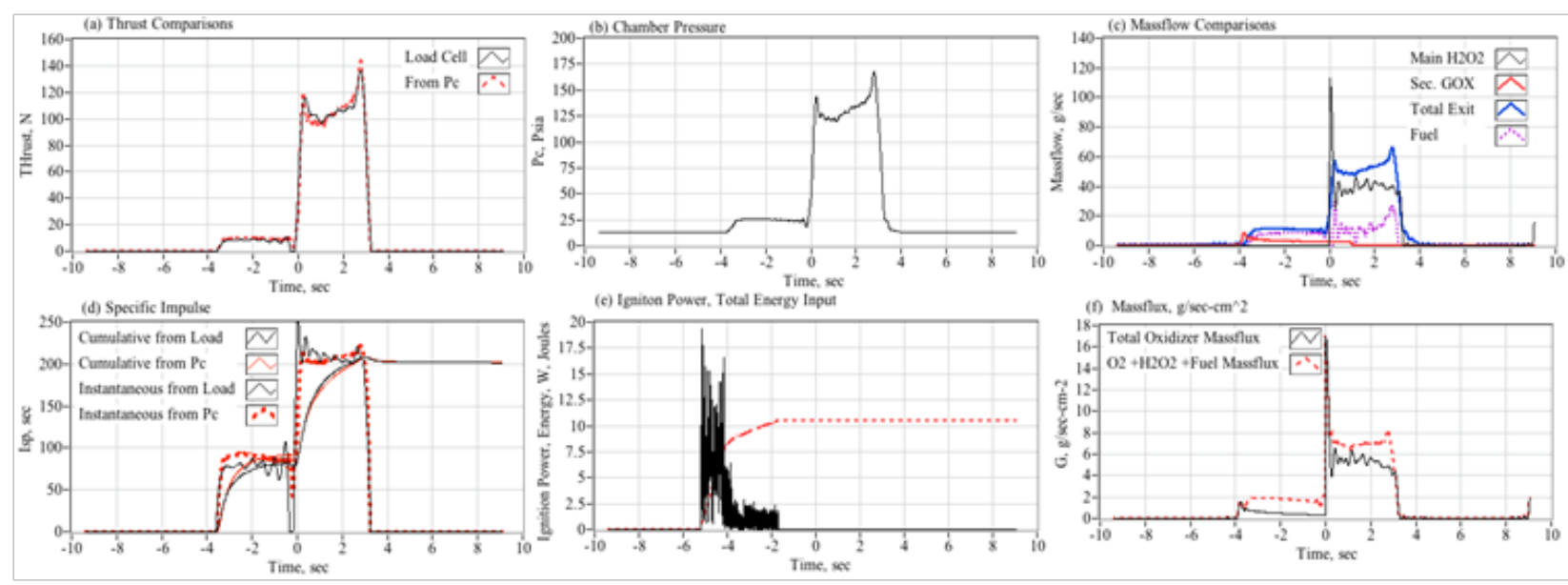

Figure 35 Preliminary hot-fire test using $87 \% \mathrm{H}_{2} \mathrm{O}_{2}$ with silver catbed augmenting GOX arc-ignition, pre-heat set at full wattage level. 


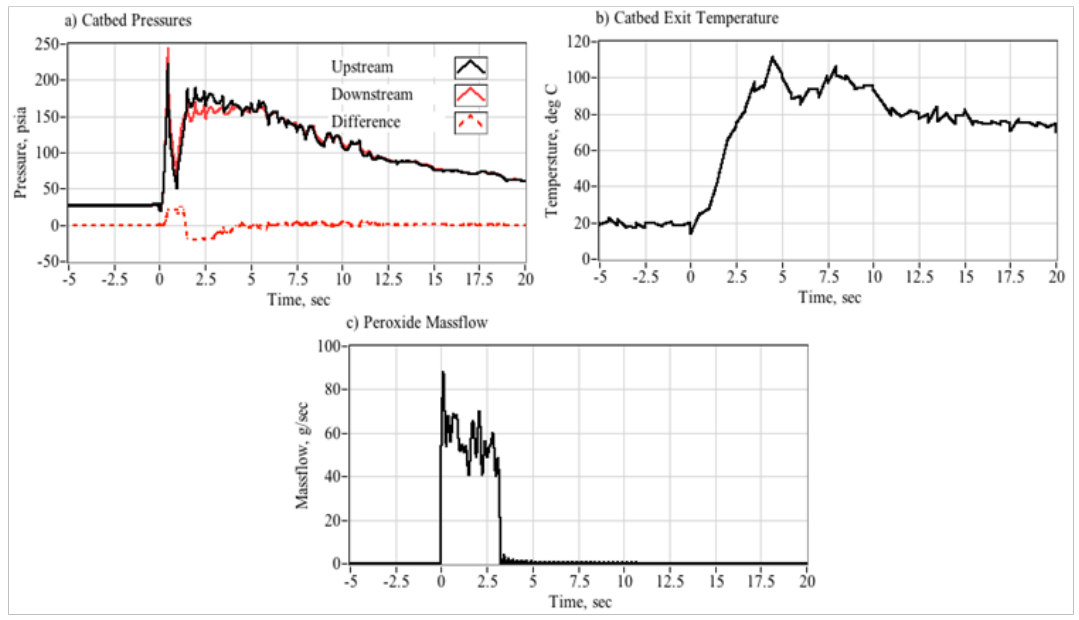

Figure 36 Initial $\mathrm{MnO}_{2}$ warm flow, pressure, temperature, massflow.

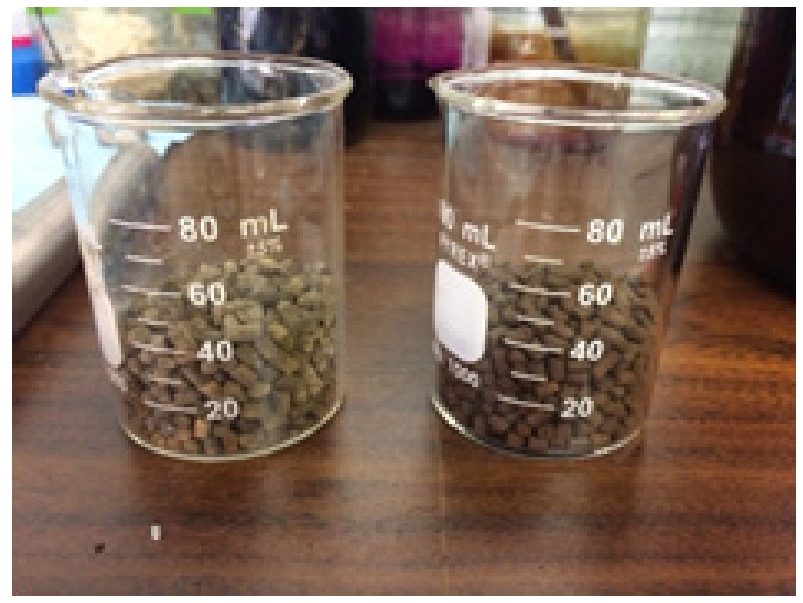

Figure 37 Pellets after warm firings (left), fresh pellets before firing (right).

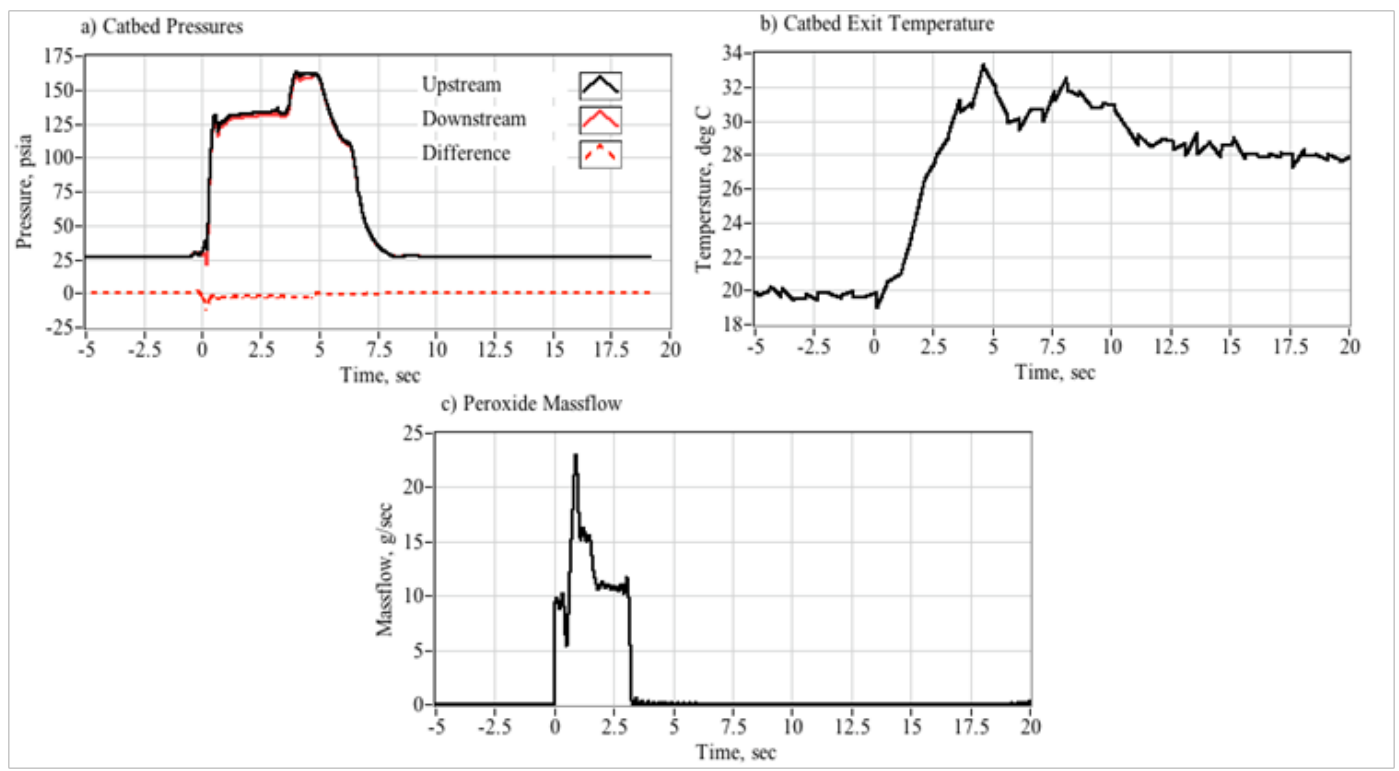

Figure 38 Repeat $\mathrm{MnO}_{2}$ warm flow, pressure, temperature, massflow across catbed, $80 \%$ peroxide solution. 


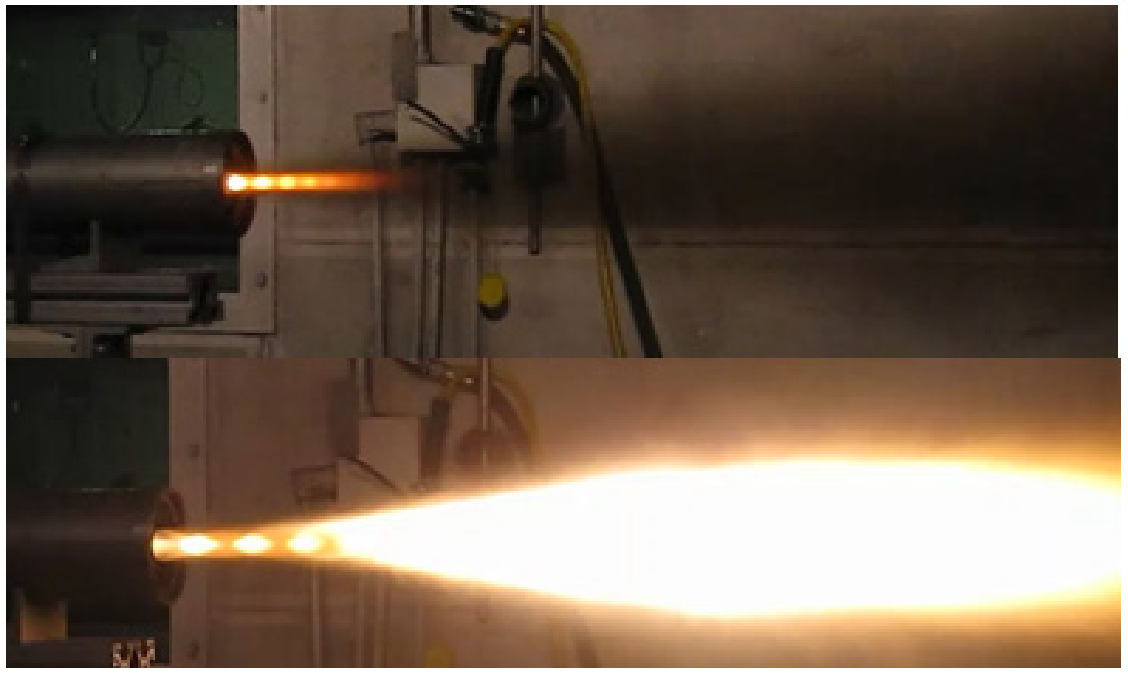

Figure 39 Initial and steady state burn images, arc-ignition with $\mathrm{MnO}_{2}$ catbed augmentation.
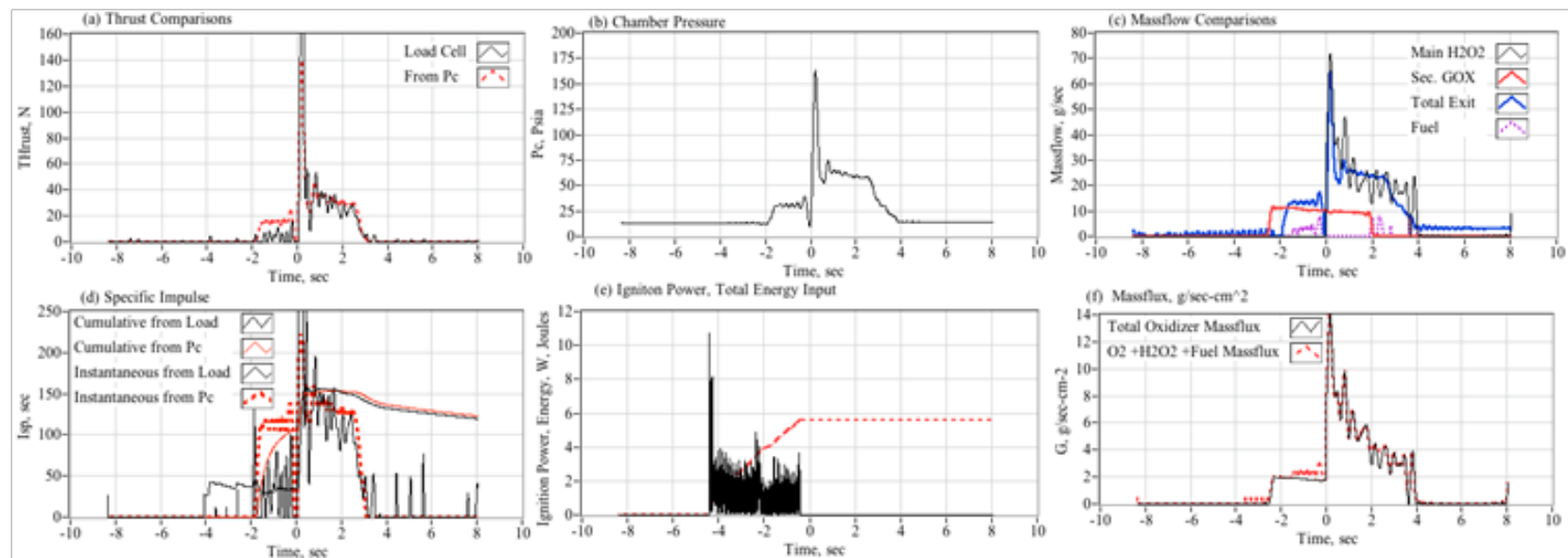

Figure 40 Response time history of first $\mathrm{MnO}_{2}$ augmentation, arc ignition hot fire test, $88 \%$ peroxide concentration.

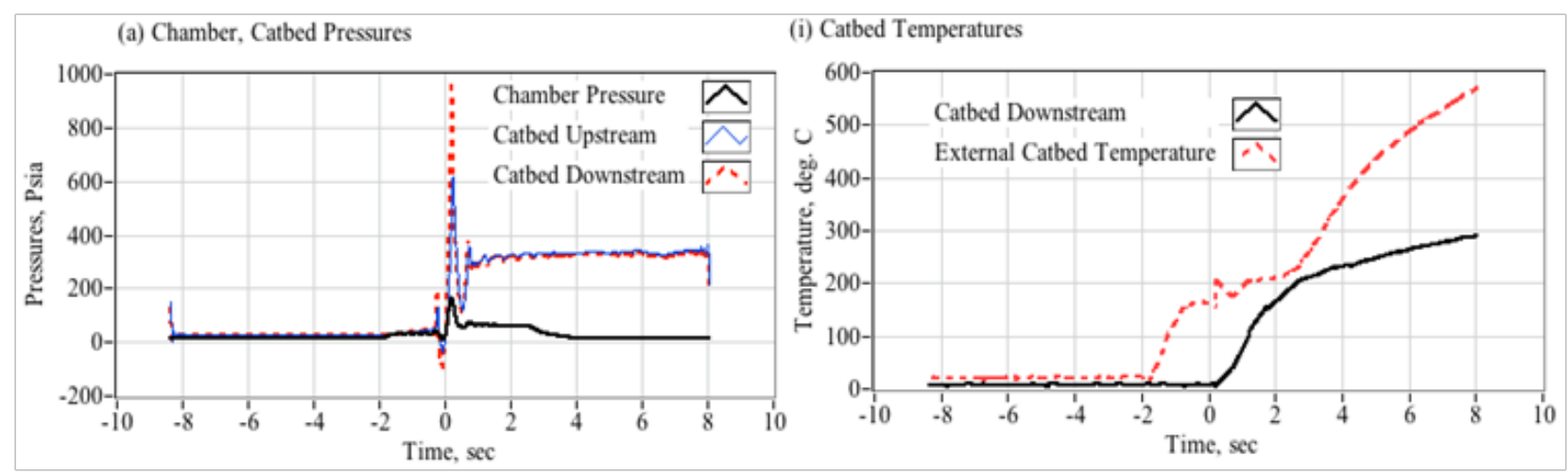

Figure $4 \mathrm{I} \mathrm{MnO}_{2}$ catbed pressure and temperature traces showing flow path blockage due to crushed alumina pellets. 


\section{Conclusion}

The authors have a prototype hybrid rocket that uses $85 \%-90 \%$ hydrogen peroxide solution and 3-D printed ABS as propellants. In the original system design the peroxide catalyst bed was completely removed and a patented arc-ignition system thermally ignited the propellants. The thermal ignition system was effective but resulted in a combustion latency of approximately 1 -second, reducing overall performance and allowing for significant variability in the delivered total impulse. This work investigates whether adding a small catalyst pack for ignition augmentation can eliminate or significantly reduce the observed ignition latency and improve overall system performance. Noble-metal catalysts have long been the "go-to" technology for monopropellant applications. Unfortunately, noble-metal catalyst has several known issues. For example, the raw material required to build even a moderate massflow catalyst bed from noble metals can easily exceed one or more thousands of dollars. Because each catalyst bed is individually hand-crafted and assembled in a one-off manner; manufacturing labor costs add significantly to the system cost. Catalyst beds fabricated from noble metals are extremely heavy and contribute nothing to the propulsive mass of the system.

Noble metal catalyst beds also have significant operational durability issues. Because the catalytic activity of the catalyst depends upon the available surface area indirect contact with the solution, oxidization due heating generally reduces the effectiveness of the catalyst bed. Similarly, stabilizers like tin salts, phosphates, nitrates, added to preserve the peroxide solution have the effect of reducing the catalyst surface area, and may in effect "poison" a working catbed rendering it ineffective. When working with higher concentrations of peroxide, the decomposition temperature can reach or even exceed the melting temperature of the noble-metal catalyst material. The result is a long term deforming, sintering, and potentially plugging the flow path. All these lead to a decrease in the performance and eventual failure of the catalyst bed. This lack of durability further adds to the costs of using silver or platinum in the catbed design.

Thus, in light of the aforementioned drawbacks with silver catalysts, and considering that the desired objective is to develop a simple, light -weight, inexpensive catbed design that will act to "assist" the primary arc-ignition system; there are clearly a variety of options that can be reconsidered. Because the catalyst is not required to fully decompose the injected peroxide stream, industry standard silver catalysts used for mono-propellants can be replaced with significantly lower-cost materials. This paper has identified wellknown and existing material alternatives, and considered how those alternatives can be best adapted to meet the stated project objectives. For this study two different augmentation catalyst designs were down-selected for hot-fire testing. The first design was based on a traditional compressed silver screen stack supported by stainless steel baffles. This traditional design was previously used by Runckel et al., ${ }^{20}$ with $98 \%$ monopropellant peroxide. The second design uses the same external form factor, but replaces the silver-screen stack with $\alpha$-alumna pellets impregnated with manganese dioxide.

A series of baseline tests were performed to assess the comparative reactivity of silver screen with the $\mathrm{MnO}_{2}$ pellets. These tests identified a nitric acid treatment as the best method for pre-activating of the silver screens. The samarium nitrate treatment recommended by Runckel was generally found to be ineffective for the $90 \%$ and lower peroxide concentrations preferred for hybrid applications. The
$\mathrm{MnO}_{2}$ pellets were found to be $20-25 \%$ more effective than the silver screen catalysts. Samarium nitrate treatments are not recommended for medium grade peroxide applications. Thus far results obtained via hot fire testing of the silver screens augmentation catalyst were moderately successful. Although the Runckel silver-screen catbed was unable to operate as a stand-alone ignition unit; it was able to successfully operate as an augment catalyst. The tests demonstrate that, when preheated, the silver catalysts effectively remove the arcignition latency and significantly improve the total specific impulse of the motor. This benefit come; however, at a steep energy price. Heating the catbed stack to an operating temperature of $400^{\circ} \mathrm{C}$ requires approximately $180 \mathrm{~kJ}$. This consumed-energy value is nearly 5 orders of magnitude greater than the required input energy or the arc ignition system.

The two burns performed to date using the $\mathrm{MnO}_{2}$ pellet catalyst have been only marginally successful. For both burns the ignition appears to proceed normally with a sharp initial rise in chamber pressure and very little pressure rise latency. However, shortly after full ignition there is a significant drop off in chamber pressure, thrust, and oxidizer massflow. Post-test examination of the catbed revealed the cause of this anomalous behavior. Once the catbed housing bed was opened the residue of multiple crushed pellets was discovered. This debris had the effect of plugging the catbed flow path, starving off the peroxide flow to the combustion chamber. This behavior has occurred with both hot fire tests, and is indicative of a structural design flaw. On a positive note, before additional testing is performed the support $\alpha$-alumina material must be replaced with a structurally stronger alternative. A complete redesign of the catalyst bed support and housing is being performed. Literature research has shown that the mechanical strength of the pellets can be improved with lanthanum doping. Also using a titanium dioxide support material to replace could prove promising and only add moderate expense. Methods for impregnating the fuel material itself with $\mathrm{MnO}_{2}$ will also be investigated.

\section{Acknowledgements}

None.

\section{Conflict of interest}

Authors declare that there is no conflict of interest.

\section{References}

1. Anon. Hazard Analysis of Commercial Space Transportation; Vol. 1: Operations, Vol. 2: Hazards, vol. 3: Risk Analysis. U.S. Dept. of Transportation, PB93-199040, Accession No. 00620693; 1988. 274 p.

2. Whitmore SA, Inkley NR, Merkley DP, et al. Development of a PowerEfficient, Restart-Capable Arc Ignitor for Hybrid Rockets. Journal of Propulsion and Power. 2015;31(6):1739-1749.

3. Whitmore SA, Chamberlain BL. Consumable Spacecraft Structures with Integrated, 3-D Printed ABS Thrusters. 53rd AIAA/SAE/ASEE Joint Propulsion Conference; 2017.

4. Whitmore SA. Three-Dimensional Printing of "Green" Fuels for LowCost Small Spacecraft Propulsion Systems. Journal of Spacecraft and Rockets. 2017;54(6):1-14.

5. Whitmore SA. Advantages of Using Additive manufacturing to Build "Green" Fuels for Hybrid Propulsion. International Journal of Astronautics an Aeronautical Engineering. 2017;2(1):1-12. 
6. Whitmore SA, Merkley DP. Arc-Ignition of an $80 \%$ Hydrogen Peroxide/ ABS Hybrid Rocket System. 53rd AIAA/SAE/ASEE Joint Propulsion Conference; 2017.

7. Whitmore SA. Additively Manufactured Acrylonitrile-ButadieneStyrene-Nitrous-Oxide Hybrid Rocket Motor with Electrostatic Igniter Journal of Propulsion and Power. 2015;31(4):1217-1220.

8. Whitmore SA, Merkley SL, Tonc L, et al. Survey of Selected Additively Manufactured Propellants for Arc Ignition of Hybrid Rockets. Journal of Propulsion and Power. 2016;32(6):1494-1504.

9. Whitmore SA, Peterson ZW, Eilers SD. Comparing Hydroxyl Terminated Polybutadiene and Acrylonitrile Butadiene Styrene as Hybrid Rocket Fuels. Journal of Propulsion and Power. 2013;29(3):582-592.

10. Whitmore SA. Additively Manufactured Acrylonitrile-ButadieneStyrene-Nitrous-Oxide Hybrid Rocket Motor with Electrostatic Igniter Journal of Propulsion and Power. 2015;31(4):1217-1220.

11. Whitmore SA, Inkley NR, Merkley DP, et al. Development of a PowerEfficient, Restart-Capable Arc Ignitor for Hybrid Rockets. Journal of Propulsion and Power. 2015;31(6):1739-1749.

12. Whitmore SA. Three-Dimensional Printing of "Green" Fuels for LowCost Small Spacecraft Propulsion Systems. Journal of Spacecraft and Rockets. 2018;55(1):13-26.

13. Whitmore SA, Mathias S, Harvey R. High Voltage Breakdown and Arc-Tracking Mechanism of Thermoplastics with Applications to Hybrid Rocket Arc- Ignition. 53rd AIAA/SAE/ASEE Joint Propulsion Conference; 2017.

14. Whitmore SA, Walker SD, Merkley DP, et al. High Regression Rate Hybrid Rocket Fuel Grains with Helical Port Structures. Journal of Propulsion and Power. 2015;31(6):1727-1738.

15. Anthoine J, Jean-Yves Lestrade JY, Messineo J, et al. Performances of a Multi-Pulsed Hybrid Rocket Engine Operating with Highly Concentrated Hydrogen Peroxide. 53rd AIAA/SAE/ASEE Joint Propulsion Conference; 2017.

16. Whitmore SA, Armstrong IW, Heiner MC, et al. High-Performing Hydrogen Peroxide Hybrid Rocket with 3-D Printed and Extruded ABS Fuel. 54th AIAA/SAE/ASEE Joint Propulsion Conference, AIAA Propulsion and Energy Forum; 2018.

17. Rommingen JE, Husdal J. Nammo Hybrid Rocket Propulsion TRL Improvement Program. 8th AIAA/ASME/SAE/ASEE Joint Propulsion Conference \& Exhibit, Joint Propulsion Conferences; 2012.

18. Rusek JJ. Hydrogen Peroxide for Rocket Propulsion Applications. Air Force Research Laboratory; 1996.

19. Sutton GP, Biblarz O. Chemical Rocket Propellant Performance Analysis. Rocket Propulsion Elements. 8th ed. New Jersey: Wiley; 2010. 168-170 p.

20. Runckel JF, Willis CM, Salters LB. Investigation of Catalyst Beds for 98-Percent-Concentration Hydrogen Peroxide. NASA TN D-1808; 1963.

21. Ponzo JB. Monolithic Hydrogen Peroxide Catalyst Bed Development. CASI Public Domain; 2003.

22. Rusek JJ. New Decomposition Catalysts and Characterization Techniques for Rocket-Grade Hydrogen Peroxide. Journal of Propulsion and Power. 1996;12(3):574-580.

23. Jonker WA, Mayer AE, Zandbergen BT. Development of a Rocket Engine Igniter Using the Catalytic Decomposition of Hydrogen Peroxide. Delft University; 2010.
24. Li H, Ye L, Wei X, et al. The Design and Main Performance of a Hydrogen Peroxide/Kerosene Coaxial-Swirl Injector in a Lab-Scale Rocket Engine. Aerospace and Technology. 2017;70:636-643.

25. Cervone A, Torre L, d'Agostino L, et al. Development of Hydrogen Peroxide Monopropellant Rockets. 42nd Joint Propulsion Conference \& Exhibit; 2006

26. An S, Kwon S. Scaling and Evaluation of $\mathrm{Pt} / \mathrm{Al}_{2} \mathrm{O}$ Catalytic Reactor for Hydrogen Peroxide Monopropellant Thruster. Journal of Propulsion and Power. 2009;25(5):1041-1044.

27. An S, Kwon S. Catalyst Bed Sizing of 50 Newton Hydrogen Peroxide Monopropellant Thruster. 44th Joint Propulsion Conference; 2008.

28. Su-Lim L, Choong-Won L. Performance Characteristics of Silver Catalyst Bed for Hydrogen Peroxide. Aerospace Science and Technology. 2009;13(1):12-17.

29. Kang S, Lee D, Kwon S. Lanthanum Doping for Longevity of Alumina Catalyst Bed in Hydrogen Peroxide Thruster. Aerospace Science and Technology. 2015;46:197-203.

30. An S, Jin J, Lee J, et al. Chugging Instability of $\mathrm{H}_{2} \mathrm{O}_{2}$ Monopropellant Thrusters with Reactor Aspect Ratio and Pressures. Journal of Propulsion and Power. 2011;27(2):422-427.

31. Jo S, Jang D, An S, et al. Chugging Instability of $\mathrm{H}_{2} \mathrm{O}_{2}$ Monopropellant Thrusters with Catalyst Reactivity and Support Sizes. Journal of Propulsion and Power. 2011;27(4):920-924.

32. Maia FF, Gouvea LH, Pereira LFG, et al. Development and Optimization of a Catalytic Thruster for Hydrogen Peroxide Decomposition. Journal of Aerospace Technology and Management. 2014;6(1):61-67.

33. Sorge R, Turco M, Pilone G, et al. Decomposition of Hydrogen Peroxide on $\mathrm{MnO}_{2} / \mathrm{TiO}_{2}$ Catalysts. Journal of Propulsion and Power. 2004;20(6):1069-1075.

34. Jo S. Response Characteristics of $\mathrm{H} 2 \mathrm{O} 2$ Monopropellant Thrusters with $\mathrm{MnO}_{2}$-Mixed $\mathrm{PbO}$ Catalyst. Aerospace Science and Technology, 2017;60:1-8.

35. Pirault-Roy L, Kappenstein C, Guerin M, et al. Hydrogen Peroxide Decomposition on Various Supported Catalysts Effect of Stabilizers. J Propulsion Power. 2002;18(6):1235-1241.

36. Bramanti C, Cervone A, Romeo L, et al. Experimental Characterization of Advanced Materials for the Catalytic Decomposition of Hydrogen Peroxide. Sacramento, California: 42nd Joint Propulsion Conference; 2006.

37. Chan YA, Hsu HW, Chen GB, et al. Study of Silver Catalyst Packing for a Low-thrust Hydrogen HTP Monopropellant Thruster. National Cheng Kung University, 8th Asia Pacific Conference on Combustion; 2010.

38. Munday TCF, Darbee LR, McCormick JC. Investigation of Decomposition Catalysts for 98\% Hydrogen HTP Final Report. California: Air Force Rocket Propulsion Lab Report AD \# 381 225; 1967.

39. Salahudden UP, Pranjal Nag HJ, Kandadai R. Design, Fabrication, Testing and Cost Optimization of Monopropellant Thruster. Global Journal of Researches in Engineering: D Aerospace Science. 2017;17(1):31-37.

40. Gordon S, McBride BJ. Computer Program for Calculation of Complex Chemical Equilibrium Compositions and Applications. NASA RP-1311; 1994.

41. Anon. Thermal Decomposition Measurement of ABS Resin I. Hitachi Application Brief TA-066e; 1995. 
42. Arisawa H, Brill TB. Flash Pyrolysis of Hydroxyl-Terminated Polybutadiene (HTPB) I: Analysis and Implications of the Gaseous Products. Combustion and Flame. 1996;106(1-2):131-143.

43. Schumb WC, Satterfield CN, Wentworth RL. Hydrogen Peroxide. New York: ACS Monograph, Reinhold Publishing; 1955. 250-251 p.

44. Ergun S. Fluid Flow through Packed Columns. Chemical Engineering Progresses. 1952;48(2):89-110.

45. Ergun S, Orning AA. Fluid Flow through Randomly Packed Columns and Fluidized Beds. Ind Eng Chem. 1949;41(6):1179-1184.

46. Akgiray O, Saatci AM. A New Look at Filter Backwash Hydraulics. Water Science and Technology: Water Supply. 2001;1(2):65-72.

47. Ebelke WH. Hydrogen Peroxide Handbook. AFRPL-TR-67-144; 1967.
48. Rusek JJ. New Decomposition Catalysts and Characterization Techniques for Rocket-Grade Hydrogen Peroxide. J Propulsion Power. 1996;12(3):574-580.

49. Fogler HS, Scott MN. Elements of Chemical Reaction Engineering, 5th ed. New Delhi: Prentice-Hall of India; 2016. 68-123 p.

50. Karabeyoglu MA, Stevens J, Cantwell BJ. Investigation of Feed System Coupled Low Frequency Combustion Instabilities in Hybrid Rockets. Cincinnati, OH: 43rd AIAA/ASME/SAE/ASEE Joint Propulsion Conference \& Exhibit; 2007.

51. Whitmore SA, Merkley DP. Arc-Ignition of a 70\%-85\% Hydrogen Peroxide/ABS Hybrid Rocket System. 53rd AIAA/SAE/ASEE Joint Propulsion Conference, AIAA Propulsion and Energy Forum; 2017. 